Florida International University FIU Digital Commons

\title{
An Investigation into Equations for Estimating Water Requirements and the Development of New Equations for Predicting Total Water Intake
}

Stacey L. Tannenbaum

Florida International University, stann001@fiu.edu

DOI: $10.25148 /$ etd.FI1 1040602

Follow this and additional works at: https://digitalcommons.fiu.edu/etd

\section{Recommended Citation}

Tannenbaum, Stacey L., "An Investigation into Equations for Estimating Water Requirements and the Development of New Equations for Predicting Total Water Intake" (2011). FIU Electronic Theses and Dissertations. 338.

https://digitalcommons.fiu.edu/etd/338 


\section{FLORIDA INTERNATIONAL UNIVERSITY \\ Miami, Florida}

\section{AN INVESTIGATION INTO EQUATIONS FOR ESTIMATING WATER REQUIREMENTS AND THE DEVELOPMENT OF NEW EQUATIONS FOR PREDICTING TOTAL WATER INTAKE}

A dissertation submitted in partial fulfillment of the requirements for the degree of DOCTOR OF PHILOSOPHY in DIETETICS AND NUTRITION by

Stacey L. Tannenbaum 
To: Interim Dean Michele Ciccazzo

R. Stempel College of Public Health and Social Work

This dissertation, written by Stacey L. Tannenbaum, and entitled An Investigation into Equations for Estimating Water Requirements and the Development of New Equations for Predicting Total Water Intake, having been approved in respect to style and intellectual content, is referred to you for judgment.

We have read this dissertation and recommend that it be approved.

Theophile Niyonsenga

Valerie George

Evelyn B. Enrione

Victoria Castellanos, Major Professor

Date of Defense: March 8, 2011

The dissertation of Stacey L. Tannenbaum is approved.

Interim Dean Michele Ciccazzo

R. Stempel College of Public Health and Social Work

Interim Dean Kevin O'Shea

University Graduate School

Florida International University, 2011 


\section{DEDICATION}

I dedicate this dissertation to my husband, Scott David Tannenbaum, MD. His faith in me and in my ability to attain my lifelong dream was unyielding. He was always available to bond together pieces of me that had somehow become unglued throughout this journey. He has strengthened my confidence in our ability to work as a team of solid support for one another. I would not have made it through this process without his unconditional love and devotion to me.

I would also like to dedicate this dissertation to my children, Jared and Ava who are the other loves of my life. It is usually the parent who is a role model and teacher to their kids about life lessons and lifelong learning. This was very true for my children as well. However, I have also learned a great deal about life and what is truly important in life through the eyes of my children. They have grounded me, improved me, and have spawned an immeasurable interwoven branch of permanent stability based on mutual respect and solid love. For this I shall always be grateful.

Lastly, I would like to dedicate this dissertation to my beloved father from whom I learned to work to my maximal capacity. I have lived my life with his philosophy in my heart and I have thought of him often throughout this process. He taught me that when the going gets tough, the tough get going. He taught me not to take no for an answer and never to quit. He gave me strength to get out of harm's way. My dad's favorite adage was, and this too shall pass. Although my dad has passed and is currently beyond harm's way, he remains a solid force in my life. Dad - the grass may well be greener on the other side, but if you are on that side that is where I hope to meet up with you again someday. 


\section{ACKNOWLEDGMENTS}

I wish to thank the faculty, staff, and all of those individuals who provided support and guidance throughout the doctoral process. I am especially grateful to Dr. Fatma Huffman who was extremely supportive and gave me enormous encouragement from the very beginning to the very end of this process. I want to thank the Florida International University Graduate School for awarding me the Dissertation Evidence Acquisition Fellowship and the Dissertation Year Fellowship. I want to also thank the American Dietetic Association Foundation for awarding me a most generous scholarship.

I would like to thank my committee. First, I wish to thank my advisor and mentor, Dr. Victoria Castellanos who spent countless hours working with me first as a student and then as a colleague and friend. Our weekly meetings gave me direction and shaped me into a tenacious scientist. Next, I want to thank Dr. Valerie George who spent myriad hours working with me as an invaluable member of my committee. She was extremely honest and supportive to me throughout my studies and especially during some difficult times. I would also like to thank Dr. Theophile Niyonsenga was also a very important member of my committee. He assisted me with some difficult statistical questions and his help was precious and highly appreciated. Lastly, I want to thank Dr Evelyn Enrione.

I would also like to acknowledge Dr. Kristopher Arheart who taught me SAS survey code. He believed in me before he even knew me. His willingness to always be available to me was completely unselfish. He was extremely generous with his time and received nothing from me but my gratitude. I am greatly in his debt for this help. 


\author{
ABSTRACT OF THE DISSERTATION \\ AN INVESTIGATION INTO EQUATIONS FOR ESTIMATING WATER \\ REQUIREMENTS AND THE DEVELOPMENT OF NEW EQUATIONS FOR \\ PREDICTING TOTAL WATER INTAKE \\ by
}

Stacey L. Tannenbaum

Florida International University, 2011

Miami, Florida

Professor Victoria Castellanos, Major Professor

The primary purpose of this study was to investigate agreement among five equations by which clinicians estimate water requirements (EWR) and to determine how well these equations predict total water intake (TWI). The Institute of Medicine has used TWI as a measure of water requirements. A secondary goal of this study was to develop practical equations to predict TWI. These equations could then be considered accurate predictors of an individual's water requirement.

Regressions were performed to determine agreement between the five equations and between the five equations and TWI using NHANES 1999-2004. The criteria for agreement was 1) strong correlation coefficients between all comparisons and 2) regression line that was not significantly different when compared to the line of equality $(\mathrm{x}=\mathrm{y})$ i.e., the $95 \% \mathrm{CI}$ of the slope and intercept must include one and zero, respectively. Correlations were performed to determine association between fat-free mass (FFM) and TWI. Clinically significant variables were selected to build equations for predicting TWI. 
All analyses were performed with SAS software and were weighted to account for the complex survey design and for oversampling.

Results showed that the five EWR equations were strongly correlated but did not agree with each other. Further, the EWR equations were all weakly associated to TWI and lacked agreement with TWI. The strongest agreement between the NRC equation and TWI explained only $8.1 \%$ of the variability of TWI. Fat-free mass was positively correlated to TWI. Two models were created to predict TWI. Both models included the variables, race/ethnicity, kcals, age, and height, but one model also included FFM and gender. The other model included BMI and osmolality. Neither model accounted for more than $28 \%$ of the variability of TWI. These results provide evidence that estimates of water requirements would vary depending upon which EWR equation was selected by the clinician. None of the existing EWR equations predicted TWI, nor could a prediction equation be created which explained a satisfactory amount of variance in TWI. A good estimate of water requirements may not be predicted by TWI. Future research should focus on using more valid measures to predict water requirements. 


\section{TABLE OF CONTENTS}

CHAPTER

PAGE

I.

INTRODUCTION

Objectives 4

Significance $\quad 4$

$\begin{array}{ll}\text { Specific aims and hypotheses } & 7\end{array}$

II. $\quad$ LITERATURE REVIEW 9

Overview 9

Physiological functions of water 9

Water regulation: normal and abnormal circumstances 11

Water balance $\quad 22$

Water requirements: challenges and factors $\quad 30$

Estimating water requirements $\quad 42$

Conclusion $\quad 52$

III. $\quad$ METHODOLOGY 54

Overview $\quad 54$

Sample and description of datasets $\quad 54$

Sample weight variables $\quad 56$

Primary sampling units (PSU) and stratification variables $\quad 58$

Data management and preparation $\quad 59$

$\begin{array}{ll}\text { Missing data } & 60\end{array}$

Eligibility criteria $\quad 60$

Variables for determination of inclusion/exclusion criteria 61

Outcome variable 63

Potential predictor variables $\quad 64$

Other predictors and potential confounding variables 65

$\begin{array}{ll}\text { Statistical analyses } & 69\end{array}$

$\begin{array}{ll}\text { Inferential analysis and assumptions } & 70\end{array}$

$\begin{array}{ll}\text { Model criteria and strategy } & 79\end{array}$

IV. $\quad$ RESULTS 83

Overview $\quad 83$

Sample characteristics and demographic findings $\quad 83$

Results of specific aim $1 \quad 86$

Summary of specific aim 1 102

Results of specific aim 2 103

Summary of specific aim $2 \quad 106$

Results of specific aim 3 106

Summary of specific aim 3 107

Results of specific aim $4 \quad 108$

Variable selection 108 
Building multiple regression model A with FFM 109

Building multiple regression model B without FFM 118

Summary of specific aim $4 \quad 124$

$\begin{array}{ll}\text { Final prediction equations } & 125\end{array}$

$\begin{array}{lll}\text { V. DISCUSSION } & 126\end{array}$

Overview 126

NHANES data $\quad 129$

Aim 1 - agreement among EWR equations $\quad 130$

Aim 2 - agreement between TWI and EWR equations 135

Aim 3 - correlation of FFM and TWI 138

Aim 4 - model building to predict TWI 141

Model A (with fat-free mass) 144

Model B (without fat-free mass) 152

Water requirements using TWI as an estimate 156

Limitations 157

Recommendations for future research 162

$\begin{array}{lll}\text { VI. } & 163\end{array}$

LIST OF REFERENCES 166

$\begin{array}{ll}\text { VITA } & 185\end{array}$ 


\section{LIST OF TABLES}

TABLE

PAGE

1. $\quad$ List of commands used in SAS version 9.2 73

2. Frequencies and percentages of categorical variables 84

3. Measures of central tendency for continuous variables 85

4. Computation information for the five EWR equations 88

5a. Simple linear regression to examine the $95 \%$ CI of the slope 89

5b. Simple linear regression to examine the 95\% CI of the intercept 90

6. Correlation coefficients of estimated water intake equation and total 92 water intake equation

7a. Simple linear regression to examine the 95\% CI of the slope 104

7b. Simple linear regression to examine the 95\% CI of the intercept 104

8. Simple regression results for total water intake outcome regressed on $\quad 107$

9. Univariate analysis of numeric variables with total water intake 109

10. Test of model A effects on full regression 110

11. Full model A of estimated coefficients and significance 112

12. Model A effects on regression without weight 113

13. Model A of estimated coefficients and significance without weight 114

14. Model A effects on regression without BMI 115

15. Model A of estimated coefficients and significance without BMI 116

16. Final model A effect of regression 117

17. Final model A of estimated coefficients and significance without 118 osmolality 
18. Test of model B effects on full regression 119

19. Full model B of estimated coefficients and significance 120

20. Model B effects on regression without weight 121

21. Model B of estimated coefficients and significance without weight 122

22. Final model B effects of regression 123

23. Final model B of estimated coefficients and significance without 124 gender 


\section{LIST OF FIGURES}

FIGURE

PAGE

1. Sources of daily water intake and routes of daily water losses

2. Regression of the Linear and Adjusted equations compared to the line of equality

3. Regression of the Linear and Harris equations compared to the line of equality

4. Regression of the Linear and Mifflin equations compared to the line of equality

5. Regression of the Linear and NRC equations compared to the line of equality

6. Regression of the Adjusted and Harris equations compared to the line of equality

7. Regression of the Adjusted and Mifflin equations compared to the line of equality

8. Regression of the Adjusted and NRC equations compared to the line of equality

9. Regression of the Harris and Mifflin equations compared to the line of equality

10. Regression of the Harris and NRC equations compared to the line of equality

11. Regression of the Mifflin and NRC equations compared to the line of equality 


\section{LIST OF ACRONYMS}

ADA American Dietetic Association

AI Adequate Intake

ATP Adenosine Triphosphate

BIA Bioelectrical Impedance Analysis

BIS Bioelectrical Impedance Spectroscopy

BMI Body Mass Index

BUN Blood Urea Nitrogen

CAPI Computer-Assisted Personal Interview

CDC Center for Disease Control

CI Confidence Interval

DRI Dietary Reference Intake

EAR Estimated Average Requirement

ECF Extracellular Fluid

EWR Estimated Water Requirement

FFM Fat-Free Mass

FIF-ARS Federal Interagency Forum on Aging-Related Statistics

FNB Food and Nutrition Board

GI Gastrointestinal

GFR Glomerular Filtration Rate

ICF Intracellular Fluid

IOM Institute of Medicine

KCAL Kilocalorie 


\begin{tabular}{|c|c|}
\hline KG & Kilogram \\
\hline LBM & Lean Body Mass \\
\hline MEC & Mobile Examination Center \\
\hline MVU & Masked Variance Units \\
\hline $\mathrm{NCP}$ & Nutrition Care Process \\
\hline NCHS & National Center for Health Statistics \\
\hline NHANES & National Health and Nutrition Examination Survey \\
\hline PA & Physical Activity \\
\hline PSU & Primary Sampling Unit \\
\hline $\mathrm{RD}$ & Registered Dietitian \\
\hline RDR & Recommended Dietary Allowance \\
\hline RMR & Resting Metabolic Rate \\
\hline RMSE & Root Mean Square Error \\
\hline TBW & Total Body Water \\
\hline TEE & Total Energy Expenditure \\
\hline TWI & Total Water Intake \\
\hline US & United States \\
\hline USDA/ARS & U.S. Department of Agriculture and Agriculture Research Service \\
\hline VIF & Variance Inflation Factor \\
\hline
\end{tabular}




\section{TABLE OF TERMS}

\section{TERMS}

Backward elimination

Confounding variables

Correlation coefficient

Hochberg adjustment procedure

\section{DEFINITION}

Fitting a model by starting with all clinically relevant variables that were statistically significant in the univariate analysis at an alpha level of 0.2 . The first regression analysis will include all of the variables and then one by one a variable is removed based on which variable returns the highest $p$-value. This procedure continues until all clinically relevant variables are significant with the lowest RMSE and the highest $F$ statistic (Kleinbaum, 2008)

An extraneous variable which can combine with the independent variable and distort the outcome variable. It is extremely important to control for confounding variables because the affect on the outcome variable may be impossible to differentiate from the independent variable. Criteria to be considered a confounding variable are: 1) must have an influence on the outcome variable, 2) must be associated with the independent variables, and 3 ) must not be a mediator or intermediate step between the independent and dependent variables. It is important to control for confounding variables either through exclusion criteria or in the analysis by stratification.

A measure of association between two variables. This number is between -1 and +1 . The closer the value is to either -1 or +1 the stronger the association. A negative association (close to -1) would mean that as one variable increases, another variable decreases. A positive association (close to +1 ) means that as one variable increases, another variable increases. The closer to zero a correlation coefficient is, the weaker the association. There is no expectation of cause and effect with correlations

This is a procedure to deal with the problem of multiple comparisons. With multiple comparisons there is a greater likelihood of a chance finding because the data are being used numerous times. This could cause a type 1 error to occur. It is therefore 
Line of equality

Multicollinearity

Root mean square error (RMSE)

Transformations

Variance inflation factor (VIF) necessary to set a stricter alpha level. This procedure calls for multiplying the chosen alpha of 0.05 by the number of comparisons. For example if 10 comparisons are tested the new alpha is 0.005

The line of equality is the plotted line on a graph that would result if all values of one variable completely matched all values of the other variable $(x=y)$. The line of equality contains a 45 degree angle from a point of $y=0$ and $x=0$. It has a slope of one and an intercept of zero

Occurs when the independent variables are not truly independent of each other and share a strong linear relationship with one another. One of the assumptions of regression is that the predictor variables are truly independent of one another. If this assumption is violated it could change the nature of the model by changing the significance of one or more variables

A measure of the differences between values predicted by a model and the observed values of the model. A model is considered to be a poor or weak model when it has a higher RMSE

Transformations are performed when there is reason to believe that one or more of the basic assumptions of regression are violated. Transformations can: 1 ) stabilize the variance if the homoscedasticity (or equal variance) assumption is violated, 2) normalize the data to a normal distribution, and 3) make the data more linear if it appears to be non-linear. Examples of transformations are logarithmic and square root

This is a test to determine if multicollinearity exists in the independent variables. VIF can be calculated by making each variable the dependent variable and regressing this variable on all of the other independent variables. The $r^{2}$ is then used to calculate the VIF. VIF $=1 / 1-r^{2}$. If the VIF is $>10$ there is sufficient evidence to believe multicollinearity exists. 


\section{CHAPTER I.}

\section{INTRODUCTION}

The essential nutrient water is the largest constituent of the human body and is the major contributor to body weight (Guyton \& Hall, 2006). In order to sustain life, water must be consumed in adequate amounts. Yet information regarding water requirements lags behind that of other nutrients (Kleiner, 1999). What is known with certainty is that ingesting too little water, which leads to dehydration and volume depletion (Thomas et al., 2008), or too much water, which leads to overhydration and hyponatremia (Grandjean, Reimers, \& Buyckx, 2003), can produce life-threatening conditions. These conditions are both preventable and treatable with appropriate water intake. In the Dietary Reference Intakes (DRIs), the Institute of Medicine (IOM) discusses the importance of assessing the amount of water that is required in the body (IOM, 2005a). But what has eluded registered dietitians (RDs) and other healthcare professionals thus far is how to accurately estimate water requirements.

Unlike other nutrients, the assessment of water requirements is fraught with challenges (Grandjean et al., 2003; IOM, 2005a). Water is a unique nutrient in that there is day-to-day variation within an individual due to changes in physical activity (PA) and environmental conditions (IOM, 2005a). There are also variations between individuals; adjustments that occur in the aging process and differences in body composition between individuals may cause the requirement for water to change (Grandjean et al., 2003; Kyle et al., 2001; Zamboni et al., 2003).

The IOM has not been able to establish a Recommended Dietary Allowance (RDA) for water because of the variation both within and between individuals, but there 
is an Adequate Intake (AI) for water (Fulgoni, 2007; IOM, 2005a). The median level of TWI (from food and beverages) from a representative sample of the US population was used to set the AI. The representative sample came from the National Health and Nutrition Examination Survey III (NHANES III).

In order to maintain water balance, total water intake (TWI) must equal total water losses (IOM, 2005a). The requirement for water is equal to the quantity needed for normal physiological functioning of the body while constantly adjusting to maintain water balance (Guyton \& Hall, 2006). Additionally, water losses must be replaced within a very short period of time in order for the individual to stay in water balance.

The IOM describes two methods to estimate water requirements (IOM, 2005a). One method is a water balance study which measures how much water an individual ingests from all sources compared to that which is lost. The other method is a water turnover dilution study which measures the difference between water influx and efflux (IOM, 2005a). Even though these two methods can estimate the water requirements of individuals, thus far, it has not been possible to apply evidence-based science to estimate water requirements with an equation.

The Evidence Library of the American Dietetic Association evaluated the question "What is the best equation for estimating water requirements in those aged 19 and older?" (ADA, 2007). The Evidence Library was created to provide the best available research on various nutrition topics through an evidence-based process. This process includes an extensive review and critical appraisal of the current and past scientific literature, a quality checklist designed to rate the scientific method completed in these 
studies, and a summary of the evidence to include suggested future research in order to fill gaps in the literature.

The Evidence Library committee concluded that there was insufficient evidence to discern the best equation for estimating water requirements and noted that none of the water requirement equations commonly applied in clinical practice and cited extensively in research studies (Chidester \& Spangler, 1997; Holben, Hassell, Williams \& Helle, 1999; Feinsod, Levenson, Rapp, Beechinor, \& Liebmann, 2004) had been validated (ADA, 2007).

There have been differences in the interpretation of the existing equations to predict water requirements. Some equations include body weight in $\mathrm{kg}$ as the sole predictor of water requirements (Chidester \& Spangler, 1997; Holliday \& Segar, 1957; Juan \& Basiotis, 2002). Another equation expressed as $1 \mathrm{ml} / \mathrm{kcal}$ expended includes the same predictors as equations developed to predict energy requirements, i.e., body weight, height, age, and gender (Harris \& Benedict, 1919; Mifflin Hill, St. Jeor, Scott, Daugherty, \& Koh, 1990; IOM, 2005b). Differences among the energy requirement equations results in variations in water requirement estimates based on these equations.

The American Dietetic Association (ADA) encourages RDs in clinical practice to utilize the Nutrition Care Process (NCP) as written in the International Dietetics and Nutrition Terminology Reference Manual: Standardized Language for the Nutrition Care Process (IDNT, 2009) in order to assure the provision of consistent quality care while improving the predictability of outcomes for patients or clients (Lacey \& Pritchett, 2003; Mathieu, 2005; Writing Group of NCP, 2008). To further this goal, all of the steps in the NCP include standardized language (IDNT, 2008). Using the NCP, an RD performing an 
assessment on a patient or client for a problem with "fluid or beverage intake" would check the criteria for evaluation in the NCP documentation. Unfortunately, the NCP documentation currently states that, "no validated standard exists" as the reference standard for the water requirements of individuals (IDNT, 2009). Despite the fact that the existing equations have not been validated, they have been considered by clinicians and researchers alike as the recommended methods or standards of practice (Akner \& Floistrup, 2003; Chidester \& Spangler, 1997; Holben et al., 1999; Lipp, Lord, \& Scholer, 1999; Wotton, Crannitch, \& Munt, 2008).

\section{$\underline{\text { Objectives }}$}

The objectives of this study were to: 1) compare and determine agreement among currently used estimated water requirement equations, 2) compare and determine agreement between currently used estimated water requirement equations and total water intake, 3) determine whether fat-free mass is associated with total water intake, and 4) create and validate one or more practical equations which could improve estimations of actual water requirements (Wang et al., 1999).

\section{$\underline{\text { Significance }}$}

The ability to estimate water requirements with improved accuracy would have great impact on the field of nutrition and have public health implications. The assessment of nutrient intake is an essential role of RDs. Estimating water requirements of individuals has historically been an imprecise process of educated guesswork (ADA, 2007). The prevailing policies and actions between and among RDs regarding water 
intake recommendations and water intake advice have been confusing and have increased the uncertainty of the public (Valtin, 2002). Therefore, equations which assess the water requirements of individuals with greater accuracy would improve the care of all individuals.

Newer equations which are better suited to individual needs allow RDs opportunities to concentrate on providing optimal care by counseling individuals with evidence-based information (IDNT, 2009). Currently-used equations are unacceptable if they jeopardize the health of individuals by not taking the known differences such as age or body composition between individuals into account.

Water requirement equations that are more accurate also have public health implications. The current Dietary Guidelines for Americans do not offer specific information to the public about water intake goals (USDHHS \& USDA, 2010). The next Dietary Guidelines for Americans in 2015 could include information for water intake requirements which are appropriate for specific population groups.

It may be appropriate to include recommendations for water intake to the public which vary based on age, body size, or other factors. There would be opportunities to educate many sectors in the public realm with accurate water information as well. For example, information about water requirements that were easily available and understandable could be explained to caregivers of an older adult or the disabled. Having this information could positively affect the care given by caregivers and improve the health and wellbeing of the individuals under their charge.

Federally funded programs that provide food and beverages for particular individuals can apply these improved guidelines in the planning and implementation of 
programs to the needy. For example, the Older Americans Act Nutrition Programs such as Meals on Wheels and Congregate Meals can provide more suitable water allowances for older adults who may be at risk for dehydration. Government spending may also be affected by better understanding of water requirements. Budgetary changes and additional information for the funding of individuals who could benefit from improved dietary intake may be affected by equations that can predict the optimal water intake of certain population groups.

The increased prevalence of obesity (Ogden, Carroll, McDowell, \& Flegal, 2007) has had an immense impact on public health and the treatment for obesity. Knowledge of how much water is required for the overweight and obese can aid in treatment plans. For example, the diet industry promotes dietary weight loss plans without specific knowledge of the appropriate amount of water to suggest for their overweight and obese clientele. This has traditionally led to dietary myths about the appropriate amount of water to include in all diets (Valtin, 2002) and inaccurate advice about water intake for those trying to lose weight (Boschmann et al., 2003).

Water requirement equations that are proven to be accurate for free-living individuals could also be tested on those who are institutionalized to determine their applicability. The amount of total water to provide individuals in psychiatric institutions, prisons, as well as long-term care (LTC) facilities and nursing homes could be tested with these equations to improve the health and care in these alternative and healthcare settings. This type of information for these types of settings could have enormous repercussions.

For example, the ability to assess the water requirements for residents in the LTC setting is vital to their ongoing care (Sloane, Ivey, Helton, Barrick, \& Cerna, 2008). The 
provision and/or consumption of inadequate amounts of water may contribute to decubitus ulcers, urinary tract infections, constipation, and dehydration (Castellanos, Silver, Gallagher-Allred, \& Smith, 2003). Dehydration results in such disastrous outcomes for the resident that it is considered a sentinel event. Significant negative ramifications occur with overestimating water requirements in the LTC setting. For example, individuals in LTC must be provided with beverages and other sources of water adequate to meet their assessed water needs (Thomas et al., 2008). When total water intake is determined to be below assessed needs, in-depth clinical assessments are required to determine whether actual needs are being met. In these cases, clinicians may need to perform physical examinations to look for signs and symptoms of dehydration and to monitor blood values such as plasma osmolality (Thomas et al., 2008). These assessments, and the extensive documentation in the medical record that accompany them, require time and resources that could be better utilized for optimal patient care.

The final goal of this study was to have an evidence-based improvement in the knowledge of the nutrient requirement for water. This type of information could advance the science of nutrition and add to public awareness regarding the appropriate amount of water intake, ultimately improving health-related outcomes.

$\underline{\text { Specific aims and hypotheses }}$

Specific aim 1: To determine agreement among existing equations used to estimate water requirements of adults using the continuous NHANES 1999-2004 datasets. 
Hypothesis 1: Estimates of water requirements among existing weight-based and energy-based equations will lack agreement with each other using the continuous NHANES 1999-2004 datasets.

Specific aim 2: To determine agreement between existing equations used to estimate water requirements of adults and total water intake of adults using the continuous NHANES 1999-2004 datasets.

Hypothesis 2: Estimates of water requirements derived from commonly used weight-based equations and energy-based will lack agreement with total water intake data from the continuous NHANES 1999-2004 datasets.

Specific aim 3: To determine the correlation between fat-free mass and total water intake using the continuous NHANES 1999-2004 datasets across the range of BMI.

Hypothesis 3: Bioelectrical impedance analysis (BIA) measures of fat-free mass and total water intake using the continuous NHANES 1999-2004 datasets will be positively correlated across the range of BMI.

Specific aim 4: To derive and validate one or more practical equations for predicting total water intake using the continuous NHANES 1999-2004 datasets. 


\section{CHAPTER II.}

\section{LITERATURE REVIEW}

\section{$\underline{\text { Overview }}$}

The essential nutrient water is so unique that determining water requirements poses distinct challenges. There are numerous life-supporting functions of water in the body (Jequier \& Constant, 2010). The IOM describes water balance as a process which is tightly regulated through physiological mechanisms in order to maintain homeostasis in the body (IOM, 2005a). Further, water balance must be upheld despite constant challenges such as extreme temperatures or heavy physical activities (Sawka, Cheuvront, \& Carter, 2005). Additionally, the water requirements of adults may differ by age (Schols, DeGroot, Van Der Cammen, \& Olde Rikkert, 2009) and body mass index (BMI) groups (Mingrone et al., 2001). Thus, assessing the amount of water needed to maintain homeostasis is tricky and complicated. The best that can be expected is not an actual water requirement but an equation that will yield a good estimation of an individual's water requirement. The equations that are currently in use should be tested for validity and, if they do not have a strong predictive value, attempts should be made to improve upon these equations.

\section{$\underline{\text { Physiological functions of water }}$}

Water has an enormity of life-supporting functions (Grandjean et al., 2003; Jequier \& Constant, 2010). Although water is located throughout the body and in all body tissue, it is more highly concentrated in areas which have higher metabolic demands as it is the main solvent and participant of chemical reactions occurring in the body (IOM, 
2005a; Jequier \& Constant 2010; Wenhold \& Faber, 2009). Cellular integrity is maintained through hydration of the cellular environment and structure, electrolyte concentration, and pH (Grandjean \& Campbell, 2004; Ho, 2006).

Water is the solvent and carrier for ingested foodstuffs, gastrointestinal (GI) secretions and for the products of digestion (Guyton \& Hall, 2006; Wenhold \& Faber, 2009). Water present in the vasculature maintains the blood volume of the circulatory system, providing both nutrients and oxygen (Jequier \& Constant, 2010; Sheng, 2006). In addition, the circulatory system supplies vital organs, muscle and all other tissues with water to allow tissue perfusion and metabolic reactions (Jequier \& Constant, 2010; Sheng, 2006).

Water brings waste products to the kidneys for processing. Under hormonal regulation, an intricate system exists in the kidneys for either reabsorption or removal of water during the excretion of urea in order to maintain water balance with amazing precision (Candela \& Yucha, 2004). Moreover, within the lower GI tract a similarly impressive mechanism of controlled water reabsorption allows for delivery of sufficient water to aid in fecal elimination by softening and providing bulk to the stool (Barrett \& Raybould, 2008).

All bodily secretions and fluids, including those in muscle and bone, and mucous, cerebrospinal, and pericardial fluids, are water-based (Guyton \& Hall, 2006). Additionally, water in fluid and secretions functions to lubricate and protect fragile or easily injured tissues, including synovial fluid of joints or vitreous humor in eyes (Guyton \& Hall, 2006). 
Maintenance of normal body temperature is a critical function of water (IOM, 2005a; Wenhold \& Faber, 2009). It is through the process of evaporation of perspiration that dissipation of body heat occurs and allows for maintenance of normal body temperature (Jequier \& Constant, 2010). Without this ability to cool the body, high body temperature would begin to denature protein structure and inhibit functions that are required to maintain life.

Water regulation: normal and abnormal circumstances

Total body water (TBW) comprises approximately $60 \%$ and $50 \%$ of the weight of an adult man and woman, respectively (Jequier \& Constant, 2010; Koppen \& Stanton, 2008; Pietrobelli \& Heymsfield, 2002). The majority of body water, approximately $66 \%$ is inside the cell as intracellular fluid (ICF) while a smaller quantity, approximately $33 \%$ is located outside the cell as extracellular fluid (ECF) (Sawka et al., 2005). ECF is further broken down into several distinct compartments: 1) interstitial fluid; 2) intravascular fluid; and 3) transcellular fluid (Whitmire, 2008). The transcellular fluid is the smallest of the ECF and is usually not included in the calculations of body water percentages.

Typically the distribution of body water is compared to a reference $70 \mathrm{~kg}$ man (Feinsod et al., 2004; Sawka et al., 2005). Using the common percentages to describe water distribution in the body, the reference $70 \mathrm{~kg}$ man has 42 liters of TBW, 28 liters of ICF (66\%), and 14 liters of ECF (33\%). The majority of the ECF or about 10.8 liters $(77 \%)$ is located in the interstitium while approximately 3.2 liters $(23 \%)$ is located in the plasma. 
Another area where water can potentially be located is often referred to as the "third space" (Sheng, 2006; Whitmire, 2008). The third space does not represent an actual water compartment. "Third spacing" occurs when water that is usually found in the ECF moves into a body cavity that does not ordinarily contain fluids, e.g., the peritoneal cavity or the pericardial cavity (Whitmire, 2008). This is a symptom of a disease process and must be managed medically. When water moves into the third space it does not change the total volume of the ECF, just its location.

\section{Water movement}

Under normal circumstances, transportation of water between water compartments occurs by the actions of osmosis and hydrostatic pressure (Grandjean et al., 2004; Guyton \& Hall, 2006). Osmosis is the movement of water across a semipermeable membrane. Movement of water occurs from an area of low to high solute concentration (Louden, 2009). However, not all solutes are alike in their water-attracting ability (Grandjean et al., 2004; Guyton \& Hall, 2006).

Hydrostatic pressure is the force exerted on membranes which causes water and solute to move from an area of higher to lower pressure (Guyton \& Hall, 2006). In both osmotic and hydrostatic pressure, the goal is equalization of the concentration of effective solute or the pressure on both sides of the membrane.

Much of the terminology associated with water movement centers around osmolarity and osmolality (Thomas et al., 2008; Whitmire, 2008). Osmolarity is body water measured in volume per liter of fluid whereas osmolality is body water measured in volume per weight (Guyton \& Hall, 2006). The effective osmolality or its tonicity is the 
amount of solute measured or estimated that contributes to a difference in the osmotic gradient across the cell membrane (Whitmire, 2008). An isotonic solution has an osmolarity of 290milliosmoles/L (mOsm/L) (Whitmire). Thus, a cell placed in a hypertonic solution will shrink whereas a cell placed in a hypotonic solution will swell (Louden, 2009).

The sensation of thirst occurs when there is an increase in the tonicity of the cell such that the effective osmotic pressure in the plasma is elevated causing water to exit from the cell (Kenney \& Chiu, 2001). Osmoreceptors in the central nervous system sense this cellular dehydration. The hypothalamus increases thirst through a feedback mechanism by encouraging beverage drinking in order to restore homeostasis (Thomas et al., 2008).

The osmolality of plasma can be measured with an osmometer or can be estimated by an equation (Whitmire, 2008). Normal osmolality of plasma is 280$290 \mathrm{mOsm} / \mathrm{kg}$ (IOM, 2005a). Although other substances contribute to osmolality, the following equation is often cited in the literature (Guyton \& Hall, 2006; Whitmire, 2008; Thomas et al., 2008) includes sodium, potassium, urea, and glucose:

Plasma osmolality $=2 \times$ [sodium in milliequivalents $/ \mathrm{L}(\mathrm{mEq})+$ potassium in $\mathrm{mEq} / \mathrm{L}]+[\mathrm{BUN}$ in $\mathrm{mg} / \mathrm{deciliter}(\mathrm{dl})] / 2.8+[$ glucose in $\mathrm{mg} / \mathrm{dl}] / 18$.

In this osmolality equation only the urea (BUN) is easily permeable to the cell membrane and therefore does not contribute to effective osmolality (Thomas et al., 2008). Urea is therefore considered isosmotic to plasma; it has the same osmolarity as plasma but is not isotonic (Whitmire, 2008). Normal saline i.e. $0.9 \%$ saline is isotonic to plasma, is the main ECF cation, and has a significant effect on effective osmolality. For 
this reason, a high level of sodium in the plasma is the main cause of the shifting of water out of the ICF to the ECF (Guyton \& Hall, 2006; Louden, 2009).

When there is a low volume of water in the ECF, the baroreceptors which are located in blood vessels are involved (Guyton \& Hall, 2006). A combination of the baroreceptor's response which cause increased thirst and the kidney's response to activate the rennin-angiotensin system which causes water retention aims to restore homeostasis (Chernoff, 1994a; Kenney \& Chiu, 2001; Thomas et al., 2008).

The inappropriate and disproportional movement of water between water compartments without compensation for this loss causes disturbances in water balance (Armstrong, 2007). Disturbances in water regulation occur when there is either a deficit or overabundance of water in the body (Grandjean et al., 2003). These disturbances need to be treated to avoid serious consequences including death (Wakefield, Mentes, Holman, \& Culp, 2009).

\section{Dehydration}

There is no official definition of dehydration (Weinberg \& Minaker, 1995). Some investigators consider any loss of total body water as dehydration (Feinsod et al., 2004; Gross et al., 1992; Kavouras, 2002; Robinson \& Weber, 2004; Shireffs, 2003; Thomas et al., 2008). Others consider dehydration as a loss of body water from the intracellular and interstitial compartments (Mange et al., 1997; Thomas, Tariq, Makhdomm, Haddad, \& Moinuddin, 2004). Armstrong (2007) described dehydration more specifically as uncompensated water loss via urine, sweat, feces, and respiratory vapor. Kleiner (1999) 
and Manz and Wentz (2003) considered dehydration to be a 1\% percent or more loss of body weight resulting from fluid loss.

An official index of hydration status is currently intangible and unattainable (Armstrong, 2007; Crecelius, 2008). There are numerous measures for determining if an individual is appropriately hydrated but most of them have severe limitations (IOM, 2005a) which affect their criterion validity (Armstrong, 2007).

The purpose of measuring hydration status is to prevent or treat abnormalities in water regulation. Despite the knowledge of the dangers of dehydration, the prevalence of dehydration in the free-living and in the institutionalized population is not exactly known, and estimates of prevalence depends mostly on the definition of dehydration chosen by the investigator (Stookey, Pieper \& Cohen, 2005; Thomas et al., 2008).

The mortality rate of individuals in a recent study who were diagnosed with dehydration during a hospital stay was significantly higher than for those without this diagnosis at the 30-day and 6-month post discharge timeframe (Wakefield et al., 2009). It is important to note that the mean age in this study was 64 years of age. This finding is in agreement with a commonly-held belief that older adults have the highest prevalence of dehydration (Wakefield, Mentes, Holman \& Culp, 2008).

Sodium has a great influence on the movement of water from the ICF to the ECF space and thus increased sodium in ECF is often the cause of dehydration (Whitmire, 2008). Dehydration occurs when water output is greater than water intake and is always accompanied by an increase in plasma sodium levels or increase in effective osmolality (Guyton \& Hall, 2006). 
Indicators other than blood have been selected to diagnose dehydration (Sarhill, Mahmoud, Christie, \& Tahir, 2003; Thomas et al., 2004). These indicators include a 25 $\mathrm{mm} \mathrm{Hg}$ or more drop in customary systolic blood pressure, and/or pulse of greater than 100 beats per minute, and/or orthostasis, including a lowering of blood pressure when changing from supine to standing position, with dry mucous membranes, tongue furrows, and a change in mental status (Madea \& Lachenmeier, 2005). The consensus in the literature is that when considered altogether, alterations in blood parameters plus physical and clinical abnormalities add credence to a diagnosis of dehydration (Sarhill et al., 2004; Thomas et al., 2008).

\section{Intravascular volume depletion}

Intravascular volume depletion or volume depletion is considered a loss of fluid from the vasculature causing decreased cardiac output and tissue perfusion (Thomas et al., 2004). This is a serious condition which can occur acutely and therefore must be identified and treated quickly and appropriately for a good outcome (Guyton \& Hall, 2006). It is accepted throughout the literature that ECF volume is maintained at the expense of a hyperosmolar ICF because without adequate blood flow, organ failure and tissue death can occur (Mange et al., 1997; Thomas et al., 2004; Whitmire, 2008). Thus, although the intravascular portion of the ECF comprises only $25 \%$ of the ECF, or $8 \%$ of TBW, there are numerous physiological mechanisms in place to closely guard its volume (Kavouras, 2002).

Volume depletion and abnormal volume regulation can be lost either as isotonic or hypotonic fluid (Thomas et al., 2008). If fluid is lost isotonically, that is, an equal 
amount of water and effective osmolal fluid (mostly sodium), is lost, a shifting of fluid from the ICF to ECF will not occur as there will be no net change in effective osmotic pressure between these compartments (Thomas et al., 2008).

Hypotonic intravascular volume depletion occurs when more sodium is lost relative to water loss (Mange et al., 1997). This will promote shifting of water into the intracellular space. The electrolyte concentration in the cell will be altered and cell swelling will occur. If isotonic fluids are lost and replaced with water alone, the hypotonic vasculature will try to equilibrate with ICF by shifting fluids into cells thereby improving the homeostatic equalization between body compartments while decreasing vascular volume (Mange et al., 1997).

Hypovolemia occurs when volume depletion is occurring in all ECF and ICF space thereby causing the total volume of water in the body to decrease (McGee, Abernethy, \& Simel, 1999). In this condition this loss of water is not being replaced and thus it can also be considered both dehydration and hypotonic hypovolemia (Kenney \& Chiu, 2001; Wakefield et al., 2009). This is a severe and life-threatening situation which must be resolved quickly and appropriately. The primary goal is to return the vasculature to normal without fluid-overloading the individual (McGee et al., 1999). The secondary goal is to return the osmotic equalization between water compartments to normal.

\section{Overhydration and hyponatremia}

The other extreme of abnormal water regulation is the overconsumption of water or water intoxication which can lead to decreased sodium concentrations in the blood (Chernoff, 1994a; IOM, 2004a; Whitmire, 2008). This can occur if water consumption 
(with inadequate amounts of sodium) is faster than the kidney's capacity for its excretion (Noakes et al., 2001). The kidneys can handle a maximal excretion rate of 0.7 to $1.0 \mathrm{~L}$ per hour (IOM, 2005a; Schrier, 2006). This excess water stays in the vasculature effectively diluting the blood. This dilution causes the hyponatremia which has potentially severe and life-threatening consequences (Grandjean et al., 2003). The symptoms of overhydration and hyponatremia include shortness of breath, orthopnea, edema, increased blood pressure, tachycardia, and ultimately death if left untreated (Chernoff, 1994a; Schrier, 2006; Whitmire, 2008).

Overhydration and hyponatremia can occur for numerous reasons. Psychiatric patients exhibit this problem in psychogenic polydipsia which is compulsive water drinking (IOM, 2005a; Schrier, 2006; Valente, 2010).

Individuals who voluntarily drink enormous amounts of water in a relatively short amount of time are also at risk for overhydration of water (IOM, 2005a). This can also occur, during inappropriate or overzealous fluid replacement for hypovolemia (Whitmire, 2008). Here, the replacement of blood volume occurs with water but without using sufficient sodium. For example, this may occur in extreme athletic performance when there is excessive perspiration loss containing both water and sodium but the athlete only replaces this fluid loss with plain water (IOM, 2005a).

In older individuals who have decreased glomerular filtration rate (GFR) and decreased blood flow to the kidneys, excesses in water input can lead to water toxicity (Chernoff, 1994a). These older adults may suffer the worst consequences of water toxicity as the symptoms can easily be explained by age-related changes in mental status and hence it may go unnoticed. 


\section{Hydration status}

There is no gold standard for measuring hydration status (Armstrong, 2007). That is, no one measure of blood, urine, or other signs and symptoms can accurately and reliably assess the degree of hydration. Therefore, to improve construct validity many investigators apply more than one indicator of hydration (Grandjean et al., 2000; Kolasa, Lackey, \& Grandjean, 2009; Martin et al., 2006).

The most commonly tested index of hydration is either plasma osmolality or electrolyte changes, more specifically sodium changes (Martin et al., 2006; Noakes, Wilson, Gray, Lambert, \& Dennis, 2001; Popowski et al., 2001; Schut et al., 2005; Shirreffs, Merson, Fraser, \& Archer, 2004; Wakefield, Mentes, Diggelmann, \& Culp, 2002). The limitation of this method is that it may not be a helpful index of hydration unless water losses are greater than solute losses (IOM, 2005a). When water and solute are lost in equal amounts, the plasma osmolality will not be altered enough to be indicative of total body water losses and dehydration. When water losses occurred increases in osmolality were more sensitive than increases in sodium. In fact, the criterion of adequacy for euhydration according to the IOM is plasma or serum osmolality (IOM, 2005a).

Other indices of hydration are measuring changes in plasma volume (Armstrong et al., 2006) or body mass (Armstrong, 2005; Armstrong et al., 2006; Grandjean et al., 2003; Noakes et al., 2001; Shirreffs et al., 2004; Soo \& Naughton, 2007). There are limitations in these methods. For a given amount of total body water loss, the degree of decrease in plasma volume is highly variable between individuals and can be different in heat acclimatized individuals. Change in body mass is a crude measure of total body 
water losses. All water intake and output must be controlled carefully. This method can only be tested over a short period of time and there are many confounding variables beyond unmeasured water intake and output that must be considered with this method (e.g. unmeasured respiratory loss and sweat loss that is hidden within clothing) (IOM, 2005a).

Urinary measures to determine hydration status are also commonly tested. Examples include measuring urine specific gravity or urine osmolality (Grandjean et al., 2003; Manz \& Wentz, 2005; Martin et al., 2006; Popowski et al., 2001; Scott, Rycroft, Aspen, Chapman, \& Brown, 2004; Wakefield et al., 2002), urine volume (Grandjean et al., 2003; Scott et al., 2004; Shirreffs et al., 2004), or urine color (Armstrong, 1994; Fletcher, Slaymaker, Bodenham \& Vucevic, 1999; Mentes, Wakefield, \& Culp, 2006; Scott et al., 2004; Wakefield et al., 2002). There are gender and race/ethnicity differences in some urinary indices (e.g. urine osmolality) (Manz \& Wentz, 2003). Thus, different population groups may have physiological differences in terms of water balance. The limitations to these methods are that the first morning urine must be collected for the most valid measure of both. Other limitations include the wide range of variability of these measures especially in the presence of osmotically actively solute (e.g., increased glucose losses in individuals with poorly controlled diabetes) (IOM, 2005a).

Sometimes investigators will measure hydration status using water balance studies (Bossingham, Carnell, \& Campbell, 2005; Martin et al., 2006) or water turnover techniques (Lane et al., 1997; Raman et al., 2004). These techniques are described by the IOM as methods that can estimate water requirements (IOM, 2005a). 
There are limitations to both of these methods. The downside of the water balance study is that it is difficult and time-consuming to measure water losses from all routes and typically they are estimated and not measured (Bossingham et al., 2005). The individuals need to be in a controlled environment in order to control for confounding factors, such as, environmental and physical activity (PA) differences. The downside of the water turnover method is that a special expertise is needed in the analysis of the data. This method is cost-limiting due to the necessity of using a mass spectrometer for the analysis and the cost of the isotopes (IOM, 2005a). These factors make these methods prohibitive for everyday clinical practice.

Some investigators have investigated the sensation of thirst as a credible index of hydration (Armstrong, 2005; Kolasa et al., 2009; Scalera, 2000; Schut et al., 2005; Shirreffs et al., 2004; Vivanti, Harvey, \& Ash, 2010). The DRIs mention thirst as an indicator of hydration status (IOM, 2005a). The IOM states that individuals will ingest the total water they need based on the physiological mechanism of thirst especially if given enough time to compensate (IOM, 2005a). Reasons other than physiological thirst (i.e. behavioral or social reasons) can cause individuals to consume more beverages (IOM, 2005a).

Other investigators consider the distribution of body water spaces (Ritz et al., 2008; Schut et al., 2005; Shanholtzer \& Patterson, 2003), physical signs and symptoms (e.g. tongue furrows, skin tenting, dry mucous membranes) (Schut et al., 2005; Vivanti, Harvey, Ash, \& Battistutta, 2008), changes in body temperature (Soo \& Naughton, 2007) or distinct features in the saliva (Walsh et al., 2004; Walsh, Montague, Callow, \& Rowlands, 2004; Yoshihara et al., 2007) as indicators of hydration status. However, there 
are other reasons for physical signs and changes to occur other than body water hydration status changes (Thomas et al., 2008).

\section{Water balance}

The requirement for water is equal to the quantity needed to replace that which is lost and for normal physiological functioning of the body while constantly adjusting to maintain water balance (Sawka et al., 2005). That is, water intake must balance with losses. Moreover, conditions of living in a Western society such as PA, relative humidity, altitude, and diet composition (e.g., salt and protein intake) can also vary on a day-to-day basis and impact the amount of water lost by an individual (IOM, 2005a). These factors increase the renal solute load causing an increase in the obligatory water loss from the kidneys (IOM, 2005a).

Further, water losses must be replaced with water intake within a very short period of time in order for the individual to maintain water balance and avoid the inevitable and rapid development of dehydration (Louden, 2009).

Homeostatic mechanisms allow most healthy people to maintain water balance. Total body water is tightly regulated within $\pm 0.2 \%$ of body weight each day (IOM, 2005a). The two major mechanisms for maintaining homeostasis are the regulation of thirst, to increase intake, and the concentration of urine, to decrease excess losses of water (Thomas et al., 2008).

A significant number of people may consume more water than is actually required to maintain water balance, i.e., they will drink water beyond what is required to prevent the sensation of thirst (de Castro, 1988). This may occur because there are many 
appealing sources of dietary water (caffeinated beverages, sweet beverages, alcoholic beverages, etc) and the body can easily excrete excess water (Campbell et al., 2007).

Popkin (2010) reported that sugar-sweetened beverages more than doubled in the past 50 years. Nielsen and Popkin (2004) reported an increase in soft drinks, fruit drinks, other sweetened beverages, and alcohol consumption expressed as a percentage of energy intake between the years 1977-2001. This increased total water intake (TWI) may not solely be due to physiological thirst. Additionally, this increased amount of water intake may be well above the minimal water intake requirements needed to maintain water balance in the body.

Recommendations to the public to drink eight, 8-ounce glasses of water per day may have also contributed to water intake in excess of actual physiological need (Valtin, 2002). Further, preformed water available from food and beverages are not taken into consideration in this recommendation. Moreover, in light of the increased intake of sugarsweetened and caffeinated beverages over the last few decades, it is likely that people are consuming an excess of water intake beyond that which is lost (Popkin, 2010).

Additionally, recommendations excluded the consumption of caffeinated and alcoholic beverages from contributing to the daily TWI (Valtin, 2002). Yet, evidence which supports the contribution of caffeinated and alcoholic beverages towards hydration and as a source of water has been provided in the literature (Grandjean et al., 2000; Kant, Graubard, \& Atchison, 2009; Scott et al., 2004; Vivanti et al., 2010).

Grandjean et al. (2000) reported no change in hydration status when the TWI including caffeinated beverage intake from habitual caffeine consumers was compared to TWI from non-caffeine consumers. Alcohol has been shown to have an antidiuretic effect 
which could last anywhere from six to twelve hours post-consumption (Taivainen, Laitinen, Tahtela, Kiianmaa, \& Valimaki, 1995). Thus it is appropriate to include caffeinated and alcoholic beverage consumption as part of TWI (IOM, 2005a).

Water intake: sources of water

All sources of water are utilized by the body to replace water losses. Figure 1 depicts the main sources of daily water intake. These sources include water from the diet, metabolic production, environment, and inspired air, (Grandjean et al., 2003; Jequier \& Constant, 2010; Manz \& Wentz, 2003). There are two ways to get water from the food and beverages we consume: 1) the production of water that occurs when adenosine triphosphate (ATP) is produced from energy-containing nutrients; and 2) the absorption of preformed water in food and beverages (Sheng, 2006). In a water balance study by Bossingham et al. (2005), the water input was measured by the amount of water consumed in food and beverages as well as the metabolic production of water.

Metabolic water, a product of the electron transport chain is formed when hydrogen, pumped back into the mitochondrial matrix to trap high energy bonds as ATP combines with oxygen (Sheng, 2006). Metabolic water contributes about $9-10 \%$ of an individual's water intake (IOM, 2005a). The oxidation of 1 gram of carbohydrate, fat, protein, and alcohol will produce $0.6,1.0,0.4$, and 1.2 grams of water, respectively (Castellanos, Silver, Gallagher-Allred, \& Smith, 2003; Raman et al., 2004; Sheng, 2006).

Preformed water, i.e., water found in foods and beverages, represents about $84 \%$ of water intake (IOM, 2005a). Preformed water is determined in the laboratory by measuring the weight difference before and after food is dried (Willits, 1951). If a direct 
measure of the water content is not readily available, the preformed water content of a food can be calculated by taking the total weight of the item and subtracting the fat, carbohydrate, protein and mineral or ash content. Although information on the macronutrient content of food is usually readily available, total mineral content is rarely provided. However, minerals represent a very small part of the total weight of most foods and beverages, so failure to correct for the mineral composition results in negligible error (Bassett \& Van Alstine, 1934).

Foods consumed represent a significant source of preformed water (Grandjean et al., 2003). The amount of water in foods varies dramatically (Campbell et al., 2007). Data from the U.S. Department of Agriculture and Agriculture Research Service (USDA/ARS) show that fruits and vegetables have the highest water content of between 80 and $95 \%$. On the other end of the spectrum, crackers, dry cereals and dietary fats are $0-15 \%$ water. Meats and cheeses are in the middle at $35-70 \%$ (USDA/ARS, 2002). For a mixed diet, it is reasonable to assume that water makes up about $60 \%$ of the wet weight (Sheng, 2006).

Drinking water and other beverages are the biggest contributors to preformed water intakes (IOM, 2005a). Kant et al. (2009) reported that in a representative sample of the US population approximately $83 \%$ of total water intake was ingested as plain drinking water plus non-water beverages while food contributed the remaining $18 \%$ of TWI. Water intake from plain drinking water is highly variable. Fulgoni (2007) reported that in a nationally representative sample of the population plain water consumption is 1.8 liters/day in adults 19 years and older. This amount was much higher than was reported by Kant et al. (2009). 
Although drinking water does contain some naturally-occurring minerals, it is reasonable to assume that $1 \mathrm{ml}$ of drinking water provides $1 \mathrm{ml}$ of water and weighs 1 gram. All beverages and liquids besides water itself contain some amount of dry matter. That is, some portion of the volume/weight is comprised of carbohydrate, protein, minerals, etc. Thus, the contribution of water in beverages or liquids is less, sometimes drastically less than its total weight (IOM, 2005a). This is not an issue of bioavailability of the water in a given item, as all water is bioavailable (IOM, 2005a). This is simply a matter of the chemical composition of the item.

Under normal circumstances all preformed water is available to and usable by the body. There is a lot of water associated with food undergoing digestion partly from water in the digestive juices added to the gut throughout and along the GI tract (Barrett \& Raybould, 2008). All of the ingested and added water in the gut is absorbed with and as part of all nutrient absorption (Sheng, 2006). Thus, unless part of the ingested food fails to be absorbed in either the small or large intestine, and ends up in the feces, all ingested and associated water is also absorbed (Barrett \& Raybould, 2008). 
Figure 1. Sources of Daily Water Intake and Routes of Daily Water Losses

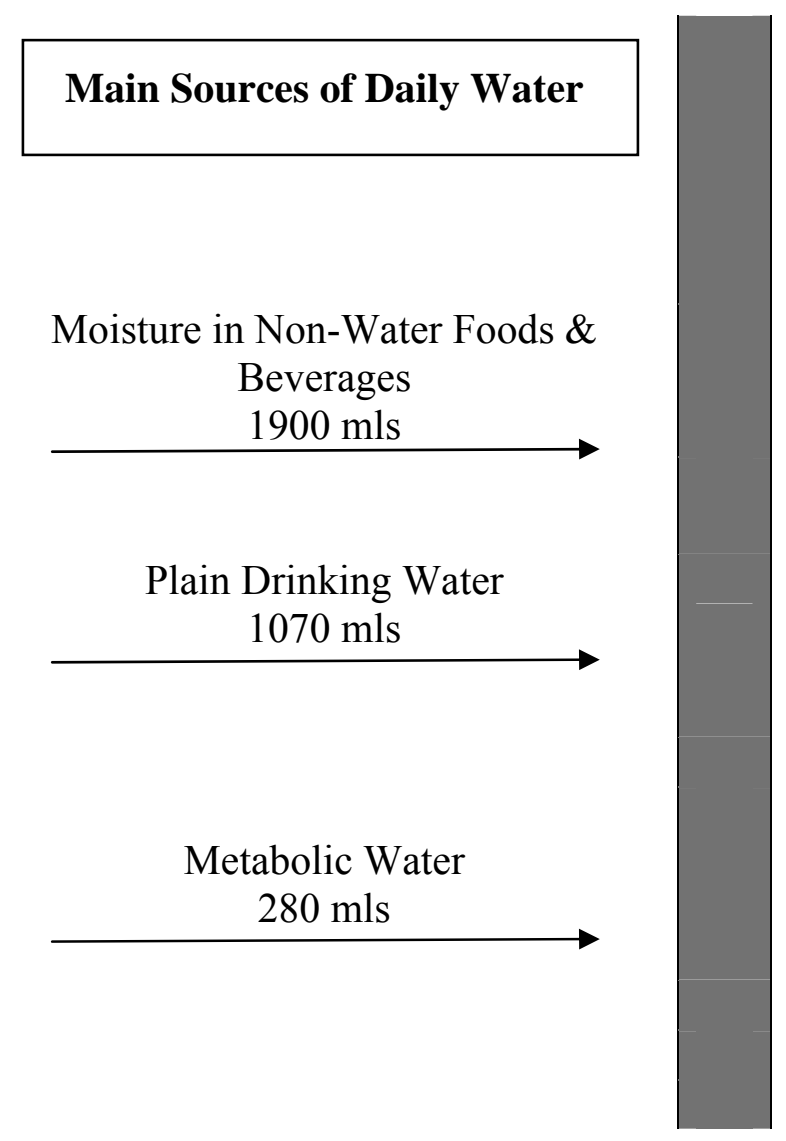

\section{Routes of Daily Water Losses}

Urine

500-2000 mls

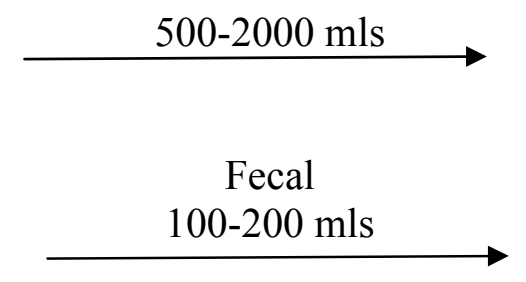

\section{Insensible Respiratory 250-350 mls}

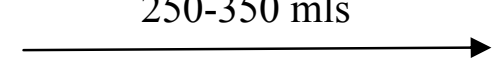

Insensible Transcutaneous

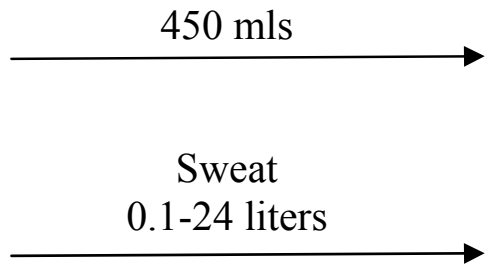

Total output $=1900+$ Sweat

Total Intake $=3250 \mathrm{mls}$

Water output: routes of loss

To maintain water balance, total water intake must equal total water losses (Jequier \& Constant, 2010). The various sources of water intake replace water losses but this replacement must occur within a very short period of time in order for the individual 
to stay in water balance (Figure 1). The routes of water losses are in urine, feces, sweat, and insensible losses through the skin and in respiration (Armstrong, 2007; IOM, 2005a).

The kidneys play the most important role in water balance by controlling the rate at which water leaves the body (Robinson \& Weber, 2004). The volume of loss through the kidneys can range between 0.5 liter/day (minimum or obligatory output) to upwards of 20 liter/day (maximal output) depending on the amount that is needed to maintain homeostasis (IOM, 2005a; Kolasa et al., 2009). Thus individuals who are dehydrated would output significantly less urine (in a more concentrated form) than those who are euhydrated (Sansevero, 1997). Individuals who are overhydrated would output more urine (in a more diluted form) than those who are euhydrated (Candela \& Yucha, 2004; Schrier, 2006).

Urine is the largest route of water loss from the body. In a water balance study by Bossingham et al. (2005) and a water turnover study by Raman et al. (2004) water loss in the urine comprised the greatest percent of total body water loss than any other route.

Water must be included in urine as a solvent for the solute load which consists of all excreted electrolytes and metabolites (Guyton \& Hall, 2006). Water that is in excess of the solute load will be freely excreted by the kidneys of a healthy individual (Berl, 2008). The ability of the kidney to readily adjust the concentration of urine allows most people to maintain normal hydration over a wide range of water intakes (Sawka et al., 2005). The excretion rate through the kidneys must precisely match intake while at the same time rid the body of excess water. It is this constant adjustment and compensation in the kidneys that plays the greatest role in water balance (Berl, 2008; IOM, 2005a). 
Fecal losses in healthy adults make up only a small amount of total water losses. Normally between 100-200 ml/day is lost in the feces (IOM, 2005a; Jequier \& Constant, 2010). However, extreme fecal losses as occurs with diarrhea can be life threatening due to the enormous and unusual losses of both water and electrolytes ordinarily not lost in this manner (Kavouras, 2002). Proper repletion of water and the corrective amounts of sodium, potassium, and other electrolytes must be provided promptly.

Sweat losses of water are highly variable based on the amount and intensity of PA as well as the environmental conditions (IOM, 2005a). A sedentary individual in a temperate climate would have about $100 \mathrm{ml} /$ day of sweat loss. This can increase to upwards of 1-2 liters/hour under extreme exercise and extreme temperatures (Guyton \& Hall, 2006). Environmental factors that alter the amount of sweat losses include clothing, humidity, air motion, and ambient temperatures (IOM, 2005a).

Water losses that come through the skin and through respiration are considered insensible losses (Guyton \& Hall, 2006). These losses are continuous throughout the day and are not precisely regulated. Under normal circumstances they account for about 700ml per day (IOM, 2005a).

Insensible losses through the skin are independent of that lost as sweat (Jequier \& Constant, 2010). Unlike sweat this insensible water loss occurs by diffusion through the skin. The average amount of insensible water loss through the skin is $450 \mathrm{ml} /$ day (IOM, 2005a). In the presence of burns or bed sores, insensible water losses are increased precipitously. The burn victim must be given upward of 5 liter/day to account for the enormous evaporation rate of water from the skin as insensible losses (Guyton \& Hall, 
2006). To counteract this water loss, burn victims are usually given intravenous fluids as part of their treatment.

Insensible losses through the respiratory tract occur through evaporation from the lungs (Guyton \& Hall, 2006). Sedentary individuals average about 250-350 ml/day of respiratory water loss (Jequier \& Constant, 2010). Respiratory losses can increase upwards to $600 \mathrm{ml} /$ day in physically active individuals (IOM, 2005a).

\section{Water requirements: challenges and factors}

The assessment of water requirements is challenging. Unlike other nutrients, water is unique in that there is day-to-day variation within an individual due to changes in PA and environmental conditions (IOM, 2005a). There are also variations between individuals; changes that occur in aging (Feinsod et al., 2004) and in body weight or body composition differences (Lee \& Gallagher, 2008; Wang et al., 1999) between individuals may affect the requirements. Thus, in the assessment of water requirements these factors must be taken into consideration.

Due to the variability of water requirements amongst and between individuals, it has not been possible to determine through dietary assessment whether or not someone is meeting his/her water requirement, i.e., there is no Recommended Dietary Allowance (RDA) for water (IOM, 2005a). The RDA is set at two standard deviations from the estimated average requirement (EAR). An EAR can only be determined if sufficient scientific information is known about a nutrient that would allow the IOM to determine the amount at which half of the population would be inadequate compared to the criterion of adequacy for that nutrient (IOM, 2005a). 
The criterion of adequacy for water is serum or plasma osmolality (IOM, 2005a). Thus, for there to be an EAR for water, sufficient evidence must exist that at a certain level of water intake, half of the population would be below this criterion of adequacy at all PA levels and in all environmental conditions. Since this level of water intake cannot be known, the DRI is the Adequate Intake (AI) for water (IOM, 2005a).

Although the AI for water is not the water requirement, it is considered the recommended amount at which adequacy of TWI in most healthy people would be expected (IOM, 2005a). Further, the IOM (2005a) acknowledges that given adequate time, TWI could be an acceptable estimate of water requirements in healthy individuals.

The AI is set at the median level of total water intake for a representative sample of the US population. The AI for water was set from the 19-30 year old life stage group of both genders from NHANES III which occurred in the years 1988-1994. At the AI for water, $3.7 \mathrm{~L} /$ day for men and $2.7 \mathrm{~L} /$ day for women, none of the life stage or gender groups were below the level of the criterion of adequacy (IOM, 2005a). That is, none of these groups had a serum or plasma osmolality which indicated that they were insufficiently hydrated.

Below the AI for water, individuals may still be able to maintain normal hydration status since there is a physiological mechanism in place to accommodate wide ranges of water intake (Grandjean et al., 2003). That is, at a lower intake of water, the kidneys can concentrate the urine to allow decreased water loss from the body.

The kidneys may not be able to compensate fully for water losses due to increased PA and environmental changes (Sawka et al., 2005). Individuals who engage in PA and are exposed to elevations in temperature will lose more water in sweat and through 
insensible losses from respiration and through the skin (Grandjean et al., 2003; Guyton \& Hall, 2006). Loss of water through sweat presents the greatest amount of variability than other water losses (Grandjean et al., 2003). This is because of the enormous potential in differences within and between individuals in day to day activity levels and hot temperatures.

An individual who is very physically active in extremely hot temperatures will require vast amounts of water compared to a sedentary individual in a comfortable temperature. A study conducted by Montain, Laird, Latzka, and Sawka (1997) demonstrated increased levels of aldosterone and arginine vasopressin under conditions of both heat and intense exercise. These hormones cause the kidneys to concentrate the urine by reabsorbing water and electrolytes back into the body to maintain a normal fluid volume and prevent dehydration (Sansevero, 1997). That is, as a response to increased sweat losses from heat and PA, the body tries to adjust with a hormonal reaction to slow further water losses. This is the normal physiological response to a perturbation of homeostasis. However, the altered need for increased water is highly variable depending on the amount of water losses from varying amounts of PA coupled with or independent of the amount of heat (IOM, 2005a).

\section{Age factor}

The amount of water that is required to maintain adequate hydration is altered by age. The prevalence of dehydration is greater in older adults although there are conflicting estimates in the literature. Stookey et al. (2005) considered numerous studies looking at the prevalence of dehydration and reported that the prevalence was anywhere 
from $1-60 \%$ depending on the exact hydration index intended to measure dehydration. Vivanti et al. (2008) reported a 16.3\% prevalence of dehydration in older adults upon admission to a hospital. Further, Wakefield et al. (2009) reported a dehydration incidence rate of $3.5 \%$ in post-hospital admission older adults.

Under the assumption that individuals consume the amount of water required, age is a factor that must be considered. Physiological changes associated with the aging body may play a role in water intake, thereby impacting water requirements (Schlanger, Bailey, \& Sands, 2010). The higher incidence of chronic disease and the need for thirstaltering medications that influence water balance also need to be considered in the aging individual (IOM, 2005a; Paterna et al., 2009; Abdallah, Remington, Houde, Zhan, \& Melillo, 2009).

With aging, specific physiological changes, such as a diminished thirst response (Ferry, 2005), might make it more difficult to meet water requirements. Phillips et al. (1984) found that older adults were less thirsty and drank less water than younger individuals. In another study, Phillips, Bretherton, and Johnston (1991), again found reduced thirst in older versus younger adults after being infused with a saline solution. This decreased thirst was found to be exasperated by heat and increased exercise in older adults (Zappe, Bell, Swartzentruber, Wideman, \& Kenney, 1996).

On the other hand, other investigators found that water intake from all sources, including that within food, did not differ substantially between older and younger individuals (de Castro, 1992; Bossingham et al., 2005). Bossingham et al. explained these differences in the amount of TWI between age groups to be the result of higher insensible losses by younger versus older individuals. However, insensible losses were not 
measured by these investigators but rather calculated based on energy requirements. Since energy requirements were higher in younger individuals, the insensible losses were also higher in younger individuals.

Older adults are not a homogeneous population. A study using a 24-hour dietary recall from NHANES 1999-2002 $(n=2,054)$ reported that younger-old (those aged 60-70) consumed more total water than middle-old (75-84) or oldest-old (those $\geq 85$ ) (Zizza, Ellison, \& Wernette, 2009). And all of these groups consumed well below the AI for water. These findings bring up the question of whether the AI for water is too high for these older adults or are they just not able to meet current water requirements.

Older individuals are more likely to be on medications which induce thirst, independent of natural physiological changes (Luckey \& Parsa, 2003; Thomas et al., 2008). Diuretics, which are more commonly prescribed to older adults than to younger adults, can increase urine output and alter water balance increasing the likelihood of dehydration (Abdallah et al., 2009).

Older adults may suffer from illness, disabilities, or specific conditions which decrease the ability to ingest sufficient water in the same amounts as younger individuals (IOM, 2005a; Paterna et al., 2009). Cognitive changes which accompany increased age may play a role in decreased TWI of older adults leading to increased risk for dehydration (Abdallah et al., 2009).

Altered kidney function which is more prevalent in older individuals can affect water balance as well (Chernoff, 1994a; Sansevero, 1997; Thomas et al., 2008). There is a reduction in the glomerular filtration rate (GFR) and free water clearance due to a diminished ability to maximally concentrate the urine (Feinsod et al., 2004; Hodak, \& 
Verbalis, 2005; Luckey \& Parsa, 2003; Schlanger et al., 2010). Other kidney factors that can specifically affect older adults are the decreased activity of renin and the decreased secretion of aldosterone (Feinsod et al., 2004; Ferry, 2005) which can directly impact water balance and urine output. However, Bossingham et al. (2005) did not find any differences in urine output between older and younger men and women in a water balance study conducted on 46 individuals. In a water turnover study, Raman et al. (2004) also found no differences in urine output between older and younger women, but 60-69 year old men had higher urine output than 40-49 year old and 50-59 year old men.

The hydration of FFM in healthy older adults is not different than in healthy younger adults (Ritz, 2000). However, older individuals have less FFM and less TBW than younger individuals (Baumgartner, 2000; Feinsod et al., 2004; Ferry, 2005; Houston, Nicklas, \& Zizza, 2009; Luckey \& Parsa, 2003; Rivlin, 2007). Older adults as a group may be engaging in less PA, may have sarcopenia, or may have chronic debilitating illness and disabilities that result in muscle wasting (Houston et al., 2009).Thus, it is logical to assume that older individuals would require less water than younger individuals. In a water turnover study conducted by Raman et al. (2004) the water requirement of 70-79 year olds was found to be, on average, $86-88 \%$ of $40-49$ year olds. When adjusted for differences in FFM, these water requirements were not significantly lower than in younger adults.

Conflicting information regarding how much water older adults require is confusing. In the newest version of the tailored MyPyramid food guide for older adults by Lichtenstein, Rasmussen, Yu, Epstein, and Russell (2008) there are eight cups of water at the base of the pyramid. This graphic and the text clearly express the need for 
older adults, specifically those $\geq 70$ years to ingest eight glasses of water daily. The fact that it was strategically placed at the base of the pyramid further emphasizes the need to ingest this amount of water. This emphasis on "eight cups of water per day" is surprising given the lack of evidence that this is consistent with the water requirements and other sources of dietary water for this age group (Valtin, 2002).

Investigators studying the water requirement knowledge of nurses in long-term care facilities reported inadequate understanding by these caretakers of the basic signs and symptoms for dehydration (Armstrong-Esther, Browne, Armstrong-Esther, \& Sander, 1996). Whether older adults are free-living or living in an institutional setting, care providers need to have a better understanding of the actual water requirements of this population.

\section{Body weight, fat-free mass and body composition factors}

Since FFM is the more metabolically active tissue and it is hydrated to a greater degree than is fat tissue (Wang, 1999), it is logical to assume that individuals who have more FFM may require more water. It must be remembered that FFM does not stay the same throughout the life cycle (i.e., individuals lose FFM with age) (Baumgartner, 2000; Feinsod et al., 2004; Ferry, 2005; Houston et al., 2009; Luckey \& Parsa, 2003; Rivlin, 2007). However, if the amount of age-adjusted and body weight-adjusted FFM is determined, prediction of an individual's water requirement should be possible.

The water requirements of overweight and obese individuals are difficult to determine without a measure of FFM. Fat-free mass consists of all tissue that is not fat tissue including skeletal muscle, connective tissue, bones, organs, and water while fat 
tissue includes storage fat and essential fat (IOM, 2005b). When mobile individuals gain weight, they will increase mostly fat tissue. There will be an increase in FFM too, and the water that is associated with this FFM, as needed to move their bigger bodies. This increase is therefore from skeletal muscle mass, the most abundant muscle tissue in the body (IOM, 2005b).

However it is the organ tissue which has the highest metabolic demand on the body (IOM, 2005b) and likely has a large water component to which it is associated. During weight gain there is a relative decrease in organ mass, as organs will not increase in absolute value relative to the rest of body size. As fat tissue increases, it is associated with less water (IOM, 2005a). Therefore, it is difficult to determine the water needs of overweight or obese individuals without a measure of FFM.

FFM can be estimated using validated techniques such as bioelectrical impedance analysis (BIA) (Van Loan, 1998; Van Loan \& Mayclin, 1987) or by other validated techniques such as dual energy x-ray absorptiometry (DEXA) (Haarbo, Gotfredsen, Hassager, \& Christiansen, 1991; Svendsen, Haarbo, Hassager, \& Christiansen, 1993; Van Loan \& Mayclin, 1992). These methods require more expense, labor, and expertise than is typically available in a clinical setting.

\section{Obesity}

The increased prevalence of obesity over the last few decades has affected the water requirements of obese individuals. In free-living adult women and men overall, more than 34\% were obese in 2005-2006 (Ogden, Carroll, McDowell, \& Flegal, 2007). The prevalence of obesity in older adults is even greater. The Federal Interagency Forum 
on Aging-Related Statistics (FIF-ARS) reported that the prevalence of obesity in women and men aged $65-74$ was $37 \%$ and 33\%, respectively in 2005-2006 (FIF-ARS, 2008). These numbers were not as high in the oldest old. In women and men $\geq 75$ years, $24 \%$ and $25 \%$, respectively were obese in 2005-2006 (FIF-ARS, 2008). In a national survey conducted by the Center for Disease Control (CDC) in the years 2005-2006, nearly $69 \%$ of adults aged 60 and older were overweight or obese (CDC, 2005-2006).

The prevalence of obesity has affected the institutionalized population as well. Lapane and Resnick (2005) reported that $25 \%$ of newly admitted residents to a nursing home were obese. This was a $67 \%$ increase in obesity from the previous decade.

Larger body weight may be a factor impacting water requirements. Using the prediction equations for energy expenditure, obese individuals by virtue of their larger body weight and lean body mass, will require more kcals than leaner individuals (Harris \& Benedict, 1919; Mifflin et al., 1990; IOM, 2005b). This increase in metabolic requirements may also translate to increased water requirements.

Additionally, a mobile obese individual is likely to lose more water in the form of sweat than a normal weight individual, further increasing water losses above that of a normal weight individual. Moreover, transcutaneous water losses may also be greater in an obese versus normal individual as there is a larger body surface area in the obese individual through which these insensible losses can occur.

\section{Body mass index}

Body mass index may play a role, albeit not as strong as FFM, in the water requirements of individuals. There is an assumption associated with BMI regarding the 
proportion of fat-free to fat tissue (IOM, 2005b). At normal BMI values this proportion is not likely to deviate in most individuals, making BMI a suitable substitute for FFM. However underweight individuals will likely have a higher fat-free to fat ratio while overweight and obese will have a lower fat-free to fat ratio. Although mobile overweight and obese individuals likely need more FFM to move their body, the most metabolically active fat-free tissue (organ tissue) is relatively smaller in individuals with higher BMI values (IOM, 2005b). However, without taking a measurement of FFM, the absolute amount of FFM in these individuals is unknown (Fuller et al., 1994; Janssen et al., 2000; Lee, \& Gallagher, 2008).

Since FFM is the more metabolically active tissue and it is hydrated to a greater degree than is fat tissue (Wang, 1999), it is likely to assume that individuals who have more absolute FFM may require more water. It is also reasonable to speculate that individuals with larger amounts of adipose tissue may have a lower water requirement, on a gram $/ \mathrm{kg}$ basis, since adipose tissue has a lower water composition compared with muscle tissue (Martin, Daniel, Drinkwater, \& Clarys, 1994). Body Mass Index is based on the assumption of a specific ratio of FFM to fat mass. However, the amount of fat-free tissue of underweight, overweight, and obese individuals is not known using BMI as the measure and therefore BMI may not be an appropriate substitute for FFM in all individuals.

The relationship between TWI and BMI in the literature is contradictory. Kant et al. (2009) reported that as energy-adjusted BMI increased, TWI increased. This implies that individuals with higher BMI may require more water than those with lower BMI, regardless of the amount of FFM. On the other hand, Raman et al. (2004) demonstrated 
that BMI was not positively associated with a higher TWI in free-living adults. Thus if an estimate of TWI is equivalent to the water requirement, BMI did not appear to affect water requirements in this study.

Bioelectrical impedance analysis

Bioelectrical Impedance Analysis (BIA) is a validated reference method to analyze body composition (Patel, Matthie, Withers, Peterson, \& Zarowitz, 1994; Plank, Monk, Woollard, \& Hill, 1998; Van Loan, 1990; Van Loan, 1998; Van Loan \& Mayclin, 1987). By passing a low level electrical current through the body, a measure of the opposition to the current called the impedance allows for the calculation of an estimate of FFM. Fat-Free Mass can be computed from the TBW estimate. The ability to estimate TBW is due to the conductivity of electrolytes present in the FFM. On the other hand the lipid-rich fat mass is a poor conductor of electricity and causes resistance or opposition to the flow of the current (Pietrobelli \& Heymsfield, 2002). There is also a degree of reactance from the cell membrane which increases impedance but most of the models that predict TBW and FFM only measure resistance.

There are numerous models created in BIA estimates of body composition. In the traditional model (i.e. the method in which single frequency measures resistance and reactance) electrodes are placed on the wrist and ankle for the measurement of wholebody impedance. The assumption behind the traditional method is that the body is a perfect cylinder. In this method the fact that the body is divided into differently sized segments is not considered. Many investigators report that this method is equal or 
superior to other methods such as multifrequency BIA (Gudivaka, Schoeller, Kushner, \& Bolt, 1999; Wotton, Thomas, Cornish, \& Ward, 2000).

Multifrequency BIA which is also called bioelectrical impedance spectroscopy (BIS) is another method using impedance analysis which allows for estimates of total body water as well as extracellular and intracellular body water. This is possible using lower frequencies which do not penetrate the cell membrane as well as higher frequencies which do penetrate through the cell membrane. There are numerous approaches that can analyze the enormity of data which accompanies this type of BIA. One of these validated models for a BIS analysis in the National Health and Nutrition Examination Survey is the Cole model (Cole, 1997).

Bioelectrical impedance analysis has developed into an important tool for body composition estimates due to the numerous advantages over other techniques. BIA is relatively inexpensive, portable, easy-to-perform, non-invasive, and can be functional for different population groups (Janssen et al., 2000).

Bioelectrical impedance analysis estimates of the amount of FFM are expected to be highly associated with estimated water requirements. Fat-Free Mass is assumed to be hydrated at a constant of $73 \%$ (Wang et al., 1999). Therefore, it may be possible to consider BIS estimates of FFM to predict water requirements in most individuals. Certain population groups such as older adults may require a specific prediction equation in order to have an accurate estimate of FFM (Kyle et al., 2004; Sergi et al., 2006). Further, the Cole model utilized by NHANES is beneficial for the analysis of population groups or individuals with an abnormal fluid distribution (Cole, 1997). 
There are differences of opinions as to the ability of BIS to accurately predict FFM in obese individuals. Some investigators reported no difference in the bias due to obesity (Sartorio et al., 2005; Yamada et al., 2009). Other investigators reported that obese individuals need alternative prediction equations due to large differences in total body water distribution (Kanellakis, Kourlaba, Moschonis, Vandorou, \& Manios, 2010; Mager, Sibley, Beckman, Kellogg, \& Earthman, 2008; Steijaert, Deurenberg, Van Gaal, \& De Leeuw, 1997). These investigators reported that BIA and BIS cause an overestimate of FFM in obese individuals.

\section{Estimating water requirements}

The two methods described by the National Research Council Food and Nutrition Board to estimate the water requirements of adults are the water balance and water turnover methods (IOM, 2005a). One method, the water balance method, involves measuring the difference between TWI and total water output as described by Adolph (1933). Investigators using water balance studies to estimate the water requirements of men aged 18-24 years and a mean body weight of $72 \mathrm{~kg}$ under ambient temperature conditions reported between 2500 to $3200 \mathrm{ml}$ of water required per day (Consolazio, Matoush, Johnson, \& Daws, 1968; Greenleaf et al., 1977).

The other method, the water turnover method, involves reconciling the difference between water influx and efflux using isotope-labeled water by following the decline in the isotope activity with time (IOM, 2005a). Raman et al. (2004) conducted a water turnover study in 458 free-living men and women $40-79$ yrs with varying body weights to estimate water requirements in a temperate environment. These investigators reported the 
water requirements to be $2900-3800 \mathrm{ml}$ per day. These requirements are estimated to be higher than those from water balance studies as water turnover studies can be undertaken on individuals under normal active conditions whereas water balance studies usually need to be done in a more controlled and restricted environment (IOM, 2005a).

The water balance and water turnover methods are complex and time-costly methods which are not routinely or typically utilized for the determination of water requirements for individuals in a clinical setting. Instead easy-to-calculate and easy-toremember equations have been developed to assess the water requirements of varying population groups and for individuals across healthcare settings (Grandjean et al., 2003). None of these equations have been validated for free-living or institutionalized individuals using evidence-based guidelines (ADA, 2007).

\section{History of weight-based and energy-based equations}

There are several weight and energy-based equations that are currently being applied to estimate water requirements in clinical practice and in research studies. Although these equations are pervasive throughout the literature, there is little evidencebased science to prove their legitimacy. Further, a closer look at the origins of these equations reveals that there may have been some confusion regarding the purpose for their actual creation and for the subsequent interpretation of the investigator's findings. The energy-based equations will be examined first to clarify and organize the best explanation of both the weight and energy-based equation origins. 
The exact origin of the energy-based water requirements equation is unknown. The earliest mention of an equation to estimate water requirements was in 1933 when Adolph concluded that, "roughly a convenient liberal standard of total water intake for any mammal is $1 \mathrm{cc}$ per calorie". This is equivalent to and is more commonly referred to as the $1 \mathrm{ml} / \mathrm{kcal}$ equation (ADA, 2007).

The most notable of Adolph's evidence in support of the $1 \mathrm{ml} / \mathrm{kcal}$ equation was an estimate of the water losses of an average normal man on the basis of his body surface area and kcal requirements of the body (Adolph, 1933). For example Adolph estimated an average man would excrete an average of $100 \mathrm{ml}$ of water in feces per square meter body surface area per day and thus estimated a 90-140 ml range of fecal water loss. He made similar estimates based on growth or storage needs, urinary losses, basal extra-renal losses and sweat losses through exercise or temperature. After adding the water losses from the various "coefficients," and "making allowance for three times the basal consumption of energy," he concluded that the average healthy man would need a minimum of $3400 \mathrm{ml}$ of water per square meter of body surface area daily (Adolph, 1933).

There were many other invalid assumptions and scientific flaws in the body of work presented by Adolph (1933) that were consistent with the current thinking of the day but received a negative quality rating from the Evidence Analysis Library of the ADA (ADA, 2007). Several examples of his unscientific approach were, 1) Adolph did not reach this conclusion through direct experimental means, but through theoretical reasoning based on human and animal studies of related topics of interest, 2) Adolph combined the animal studies with human studies and although he acknowledged that 
there are substantial differences between the species and man, he made broad generalizations which applied to both, 3) the studies that Adolph reviewed did not contain an appraisal for quality or validity, 4) It would be very unlikely that Adolph's work could be reproduced based on the lack of adequate methodology information, 5) the results were not clearly presented in quantitative terms with significance levels or confidence intervals included, 6) results with limitations and biases were not considered in the conclusions, and 7) Adolph gave his opinion based on wide-ranging generalizations rather than evidence-based science.

In spite of this, the Food and Nutrition Board (FNB) included Adolph's equation in the $10^{\text {th }}$ edition of the RDA (FNB, 1989). Although there may not have been alternative equations available to include in the old RDA, in terms of today's standards, this body of work does not meet the criteria for evidence-based research (ADA, 2007). Nonetheless, this equation of $1 \mathrm{ml}$ of water/kcal has been cited as a standard method to determine water requirements numerous times in the literature and is still used today (Armstrong-Esther et al., 1996; Chidester \& Spangler, 1997; Feinsod et al., 2004; Ferry, 2005; FNB, 1989; Kayser-Jones, Schell, Porter, Barbaccia, \& Shaw, 1999).

Although Adolph's intention was for this equation to represent $1 \mathrm{ml}$ per kcal required by the body, it has been interpreted in alternative ways. For example, the reviewer's comments in the Evidence Analysis Library of the ADA (ADA, 2007) summarizes Adolph's "convenient liberal standard of total water intake" as the possible source for "fluid recommendations of $1 \mathrm{cc}$ of water per calorie consumed".

A difference in the interpretation of the simple equation (i.e. $1 \mathrm{ml}$ per kcal consumed versus expended versus required) may affect how this equation is applied 
clinically or in the scientific literature. Some investigators express the equation as 1 ml/kcal consumed (Dickerson \& Brown, 2005; Feinsod et al., 2004; Holben et al., 1999). Other investigators express the equation as simply $1 \mathrm{ml} / \mathrm{kcal}$ without any distinction of how they would determine kcals (Armstrong-Esther et al., 1996; Lipp et al., 1999) or as 1 ml/kcal expended (Ferry, 2005; Grandjean et al., 2003; Raman et al., 2004).

If individuals are in energy balance and consume the energy they expend, there would be little difference between these calculations. It has been shown, however that the amount of kcals expended or estimated to be required may not match that which is actually consumed and may be a function of differences in methodology (da Rocha, Alves, Silva, Chiesa, \& da Fonesca, 2005 ). These differences in methodology and in clinical application of this equation may in part explain discrepancies between investigators and clinicians.

A study by Chidester and Spangler (1997) is a good example of the implications of using energy required vs. energy consumed. This study compared the actual water intake of elderly individuals in an institutional setting to three "established standards" (Chidester \& Spangler, 1997). One of the standards was $1 \mathrm{ml} / \mathrm{kcal}$ energy consumed. When the analysis of energy intake was undertaken, $60 \%$ of individuals were found to be below this standard. This meant that if they consumed 2000 kcals of food they were taking significantly less than $2000 \mathrm{ml}$ of water. For example, they may have only taken in $1600 \mathrm{ml}$ of water that day. However, in actuality their energy requirements may only be $1600 \mathrm{kcal} /$ day. If $1 \mathrm{ml} / \mathrm{kcal}$ required had been used as the standard instead of $1 \mathrm{ml} / \mathrm{kcal}$ consumed, their intake may not have been considered below the standard. Other 
investigators have found comparable results when they performed studies with similar methodologies (Holben et al., 1999; Kayser-Jones et al., 1999).

The other investigators credited with developing an energy-based formula are Holliday and Segar (1957). They sought to develop a simple equation for estimating both energy and water needs in hospitalized patients of all ages on parenteral nutrition. These investigators decided that maintenance water requirements could be based on estimates of caloric expenditure. Using calculations based on previous experiments they determined that $50 \mathrm{ml} / 100 \mathrm{kcal} / \mathrm{day}$ could replace insensible water losses while $66.7 \mathrm{ml} / 100 \mathrm{kcal} / \mathrm{day}$ could replace kidney losses. Therefore, they decided that the total requirements for water would be $116.7 \mathrm{ml} / 100 \mathrm{kcal} / \mathrm{day}$. Since metabolic water could supply the 16.7 $\mathrm{ml} / 100 \mathrm{kcal} / \mathrm{day}$, the water requirements of hospitalized patients on parenteral nutrition would be $100 \mathrm{ml} / 100 \mathrm{kcal} /$ day or $1 \mathrm{ml} / \mathrm{kcal}$ required/day (Holliday \& Segar, 1957). Thus, even though this equation was developed to estimate the requirements of individuals who where ill, this equation is the same as the equation derived by Adolph (1933) 24 years earlier.

Holliday and Segar (1957) also developed a simple equation for estimating energy needs in hospitalized patients on parenteral nutrition. They developed an equation for estimating energy requirements based on their belief that there is a relationship between energy expenditure and weight. Holliday and Segar were able to plot the amount of estimated energy expended for each kg body weight. The energy expenditure was based on previous equations by Darrow and Pratt (1950). Energy requirements were then determined to be $1500 \mathrm{kcal}$ for the first $20 \mathrm{~kg}$ of body weight and then an additional 20 
$\mathrm{kcal} / \mathrm{kg}$ thereafter. The equation they developed to determine energy expenditure was 20 kcal x (kg body weight-20) $+1500 \mathrm{kcal}$.

Although this equation by Holliday and Segar (1957) was created with the intention of estimating energy requirements in hospitalized patients receiving parenteral nutrition therapy, it has over time become one of several standard weight-based equations for approximating water requirements of both healthy and sick individuals (Chidester \& Spangler, 1997; ADA, 2007). That is, the equation currently used to estimate water requirements was originally intended as an energy expenditure equation. Over time this equation became $20 \mathrm{ml} \mathrm{x} \mathrm{(kg} \mathrm{body} \mathrm{weight} \mathrm{-} \mathrm{20)}+1500 \mathrm{ml}$ (Holliday \& Segar, 1957). Other investigators have made alterations to this equation by changing the multiplier from 20 to 15 , however, explanations regarding why these alterations were made have not been provided (Grant \& DeHoog, 1999; Holben et al., 1999).

Other weight-based equations were described to estimate water requirements. Chernoff (1994b) started with $30 \mathrm{ml} / \mathrm{kg}$ body weight, but then added a minimum of 1500 $\mathrm{ml} /$ day for the purpose of assuring adequacy in water intake, especially in frail individuals. Other investigators agreed with this equation as well (Dimant, 2001; Holben et al., 1999; Juan \& Basiotis, 2002). Speculation regarding the origins of this weightbased equation includes a "rule of thumb" energy expenditure equation for healthy adults of $30 \mathrm{kcal} / \mathrm{kg}$ body weight. Although this equation has been utilized in clinical as well as research settings (Koea, Wolfe \& Shaw, 1995) the origin of this equation is also unknown. Thus, the $30 \mathrm{ml}$ of water $/ \mathrm{kg}$ body weight equation may have evolved from the logic of Adolph (1933) and Holliday and Segar (1957), i.e., that one $\mathrm{ml}$ of water is required per kcal required. 
Other investigators have also considered weight-based equations in research. The multipliers applied by these investigators ranged from 25 to $40 \mathrm{ml} / \mathrm{kg}$ body weight instead of 30 ml/kg (Akner \& Floistrup, 2003; Biesalski, Bischoff, Boehles \& Muehlhoefer, 2009; Lipp et al., 1999; Wotton et al., 2008).

\section{Problems of using equations to estimate water requirements}

Although an evidence-based equation which can estimate water requirements has not been developed and validated (ADA, 2007), there have been numerous references in the literature which state that "standard" equations do exist (Chidester \& Spangler, 1997; Holben et al., 1999; Kayser-Jones et al., 1999). The word "standard" implies a validation of these techniques has previously occurred. The lack of true standardization of water requirement equations and a call for further research into this area has been expressed in the International Dietetics and Nutrition Terminology Reference Manual (IDNT, 2009).

Chidester and Spangler (1997) studied three such "standard" equations in elderly nursing home residents to evaluate whether residents were getting sufficient water. The standards they described were: 1) $30 \mathrm{ml} / \mathrm{kg}$ body weight, 2) $1 \mathrm{ml} / \mathrm{kcal}$ energy consumed and 3) $[20 \mathrm{ml} x$ (body weight -20)] $+1500 \mathrm{ml}$. Holben et al. (1999) studied four "standards" in elderly residents which were investigated to estimate their fluid needs. The standards they tested were, 1) $30 \mathrm{ml} / \mathrm{kg}$ actual body weight, 2) $30 \mathrm{ml} / \mathrm{kg}$ actual body weight with a minimum of $1500 \mathrm{ml}, 3) 1 \mathrm{ml} / \mathrm{kcal}$ energy consumed and 4) [15 $\mathrm{ml} \mathrm{x}$ (actual body weight -20)] $+1500 \mathrm{ml}$. Thus, the standards applied by Chidester and Spangler (1997) do not exactly match those of Holben et al. (1999) and Holben et al. apparently had knowledge of a fourth standard. 
One of the problems in these studies lies in the lack of distinction between kcals required and kcals consumed. The original intent of the energy-based equation of $1 \mathrm{ml}$ of water/kcal required was to determine energy needs and apply these in the equation (Adolph, 1933; Holliday \& Segar, 1957). Unfortunately the equation that was applied by these investigators was $1 \mathrm{ml}$ of water/kcal consumed (Chidester \& Spangler, 1997; Holben et al., 1999). The amount of kcal consumed may be very different than the amount of kcal required, especially in a population of frail, elderly nursing home residents that are likely suffering from both acute and chronic illnesses.

Energy requirements can be determined using one of many energy requirement equations that have been validated against indirect calorimetry or doubly labeled water (Harris \& Benedict, 1919; Mifflin et al., 1990; IOM, 2005b). Some of the well known and thoroughly validated equations are, 1) The Harris Benedict equation (Harris) for resting metabolic rate (Harris \& Benedict, 1919),

Female: $655+9.6$ Weight $(\mathrm{kg})+1.85$ Height $(\mathrm{cm})-4.7$ Age $(\mathrm{yrs})$

Male: $66+13.8$ Weight $(\mathrm{kg})+5$ Height $(\mathrm{cm})-6.8$ Age $(\mathrm{yrs})$

2) The Mifflin St. Jeor equation (Mifflin) for resting metabolic rate (Mifflin et al., 1990),

Female: $-161+9.99$ Weight $(\mathrm{kg})+6.25$ Height $(\mathrm{cm})-4.92$ Age (yrs)

Male: $5+9.99$ Weight $(\mathrm{kg})+6.25$ Height $(\mathrm{cm})-4.92$ Age $(\mathrm{yrs})$

3) The National Research Council equation (NRC) for total energy expenditure (IOM, 2005b). [In this equation PA is the physical activity factor.]

Female: $354-6.91$ Age $(\mathrm{yrs})+9.36$ Weight $(\mathrm{kg}) * \mathrm{PA}+726$ Height $(\mathrm{m}) * \mathrm{PA}$ Male: $662-9.53$ Age +15.91 Weight $(\mathrm{kg}) * \mathrm{PA}+540$ Height $(\mathrm{m}) * \mathrm{PA}$ 
The water requirement estimation derived from an energy-based equation will vary depending on which equation is selected to estimate energy requirements. These discrepancies between the equations may be secondary to variations in the methods selected to determine energy expenditure. For example, there were differences in the populations from which equations were derived. The Mifflin equation was derived from a sample of 498 individuals which included all BMI groups (Mifflin et al., 1990). The sample contained few individuals $>80$ years. On the other hand, the Harris equation was derived from 239 individuals and only contained those in the normal BMI group (Harris $\&$ Benedict, 1919). The sample did not contain individuals $>74$ years. The NRC equation (IOM, 2005b) was derived using 400 normal-weight individuals with doubly labeled water as the gold standard for energy expenditure.

Other issues that arise when different equations are utilized to estimate water requirements is the controversy regarding whether actual or "adjusted" weight should be included in the energy-based and the weight-based equations (Krenitsky, 2005). Frankenfield et al. (2003) compared several equations which predict resting energy expenditure (REE) and found that when they incorporated adjusted body weight, there was less but still significant overestimation of energy requirements being predicted. Additionally, there is no standard or validated approach to determine "adjusted" body weight (Amato, Keating, Quercia, \& Karbonic, 1995), which is a fundamental problem with the lack of standardization of equations used by RDs in practice to estimate dietary needs of clients.

The weight-based equations are also fraught with problems related to assumptions. For example, the water requirements of weight-based equations are the 
result of the multiplication of a constant number by body weight in kilograms. This assumes that water requirements are positively correlated to weight and this would be true at all values of body weight (ADA, 2007). In actuality, it is the FFM which is hydrated to a more substantial degree than fat tissue (Fuller, Sawyer, \& Elia, 1994; Wang et al., 1999). Since overweight and obese individuals have more absolute fat tissue and fat tissue requires less water than FFM, it is logical to assume that these weight-based equations will overestimate water needs at higher body weights.

Some studies have compared values derived from the energy-based and/or weight-based equations to actual water intake (Chidester \& Spangler, 1997; Holben et al., 1999; Juan \& Bastiotos, 2002). But, there is a lack of research to show whether the energy-based and weight-based equations are comparable in what they are measuring. That is, are they measuring the same concept? The only variable in the weight-based equation is weight while the energy-based equations contain weight, age, height, gender, and physical activity (in some). The equations that have more variables may be measuring something completely different than the equation with only one variable.

\section{Conclusion}

None of the equations to estimate water requirement, which are commonly utilized in clinical practice and cited extensively in research studies and well-respected documents, has been validated (ADA, 2007). Despite this lack of proven validity and despite the lack of a standard by which to judge water needs, these equations are being used by clinicians and researchers as the standard of practice and the recommended 
methods to estimate water requirements (Chidester \& Spangler, 1997; Holben et al., 1999).

The purpose of this study was to determine if five of the equations that RDs are currently using to estimate water requirements agree with each other and with another estimate of water requirements as measured by total water intake. The degree of association between FFM and total water intake were determined within each BMI category. Two equations to estimate water requirements as measured by total water intake were created. Both equations included all clinically relevant variables but one was created with the FFM variable and the other without the FFM variable. An improvement in the prediction of water requirements using a more suitable equation was the goal. 


\section{CHAPTER III.}

\section{METHODOLOGY}

\section{$\underline{\text { Overview }}$}

This study is based on secondary data from a nationally representative survey sample (continuous NHANES 1999-2004) to assess the agreement among equations to estimate water requirements and to assess agreement between EWR equations and total water intake. In addition, this study assessed the association between fat-free mass and total water intake (TWI). New models to explain TWI with and without fat-free mass (FFM) were investigated.

\section{$\underline{\text { Sample and description of datasets }}$}

NHANES is a complex cross-sectional survey developed by the National Center for Health Statistics (NCHS) in order to collect information about the health and diet of a nationally representative sample of the non-institutionalized U.S. population (CDC, 2010f). The survey utilized a complex multi-stage probability design in order to get nationally representative samples and assure unbiased estimations of the U.S. population parameters (CDC, 2006). Additionally, because generalizations about the population were made from the data in these surveys, it was extremely important that the sample be selected in a way that accurately represented the whole population (CDC, 2006).

The sampling included four stages in which statisticians calculated the size of the sample needed to be representative of the U.S. non-institutionalized population by taking into consideration the geographic distribution and demographic characteristics of the population, such as age, gender, race/ethnicity, and income (CDC, 2006). In the first 
stage, NCHS statisticians drew a sample containing units referred to as a Primary Sampling Unit, or PSU, using probability methods. The PSUs were selected among numerous geographic areas located throughout the entire nation. These PSUs might be the size of one or more counties. In the second stage again using probability methods small sections were selected within each PSU. In the third stage, households within the smaller sections were randomly selected to be included in the survey. In the fourth and final stage, a stratified random sample of individuals was selected from within the household, based on strata that took age, gender, and race/ethnicity into account (CDC, 2006; CDC, 2010e).

The study respondents included non-Hispanic Whites as well as an oversampling of non-Hispanic Blacks, Mexican-Americans, adolescents, older individuals, and pregnant women (CDC, 2010e). The reason for oversampling of these groups was to assure their representation in the sample. In past years these particular groups had low response rates to the NHANES surveys altering the chance of having a sufficient sample size to allow for valid estimations from these groups. Thus, oversampling assured that the US population was sufficiently represented despite differences in response rates and assured unbiased national estimates. To permit the data to be adequately handled, information was supplied regarding weighting the final survey sample (CDC, 2006). Indeed, analysis of the data must incorporate the survey weights to account for the differences in response rates and missing data as well as the multi-stage probability sample design as previously described.

Survey data were first collected in the household and then in a mobile examination center (MEC) by highly trained medical staff employed by NHANES (CDC, 
2006). The survey consisted of a questionnaire, physical examination, and laboratory data collection (CDC, 2009). Every step of the data collection was fully explained to each participant. Each participant was de-identified and assigned an identification number called a sequence number in order to maintain strict confidentiality. A consent form was signed by the participant before any parts of the survey were administered.

Beginning in the year 1999, the NHANES survey changed from a periodic to an annual and continuous survey (CDC, 2006). Every two years the survey data were released on publicly accessible data files. For NHANES 1999-2004 there were 38,077 persons selected for the sample, 31,126 of those were interviewed in the household and 29,402 were examined in the MEC.

The continuous NHANES survey years utilized for all research questions in this study were 1999-2000, 2001-2002, and 2003-2004 (CDC, 2010a; CDC, 2010b; CDC, 2010c). The reason for choosing these particular datasets was that all of the variables to be tested were available in these 6 years of survey data for all research questions.

\section{$\underline{\text { Sample weight variables }}$}

Due to the nature of the sampling design, sample weight variables were created and utilized for each participant in the sample in order to assure unbiased national estimates (CDC, 2006). The sampling design was a multi-stage probability sample in which the sample was purposefully selected with the intention of attaining a representative sample (CDC, 2006). The weights were created by calculating the reciprocal of the final probability of selection for each of the stages of sampling (CDC, 
2006). This base weight was then further adjusted for post-stratification and for the rate of non-response (CDC, 2006).

Weighting of the surveys was complicated by the differences in how individual two-year survey weights were determined. The Bureau of the Census collected Census counts in the year 2000. Therefore the sampling weights for the 1999-2000 NHANES were based on prior population estimates from the Census of 1990 (CDC, 2006). The 2001-2002 and 2003-2004 NHANES surveys based the population estimates on the 2000 Census counts, making the population estimates of these cycles dissimilar. To account for these differences, the NCHS recommended that if the first two continuous NHANES cycles (1999-2000 and 2001-2002) were part of the analyses, the procedure was to consider the time period 1999-2002 as one survey and to utilize the appropriate 4 year sample weights for these four years (CDC, 2006). Each additional two-year cycle could be merged (using the associated two-year weights) to this four-year time period and then the appropriate sampling weights would need to be calculated based on the total years analyzed (CDC, 2006).

The appropriate sampling weight for the analysis is the weight associated with the variable that was collected from the smallest number of participants for that particular analysis (CDC, 2006). As an example, if a participant had demographic data collected from the household interview and body composition data collected from the MEC, the appropriate sampling weight in that analysis would be the MEC weight from the physical examination files since the variables collected from the body composition data is located in the physical examination files which contained a smaller number of participants than those from the demographic data collected in the larger household interview files. The 
sampling weights for the demographic data were located in the demographic data files for those years analyzed (CDC, 2009).

The weight variable in the present study was calculated from the variables collected from the smallest sample size for the combined datasets from NHANES 19992000, 2001-2002, and 2003-2004 (CDC, 2010a; CDC, 2010b; CDC, 2010d). A new weight variable was created that was appropriate for these analyses. The variable WTMEC6YR was created based on the four-year sampling weight, labeled by NCHS as WTMEC4YR for the files created in 1999-2002 and the two year weight variable, labeled by NCHS as WTMEC2YR for the years 2003-2004. The new variable WTMEC6YR was obtained by taking 2/3rds of WTMEC4YR for variables collected in NHANES 19992002 and 1/3rd of WTMEC2YR for variables collected in NHANES 2003-2004. The reason for taking 2/3rds and 1/3rd of the NHANES 1999-2002 and the NHANES 20032004 , respectively was that each two-year cycle was considered to be $1 / 3 \mathrm{rd}$ of the entire sample so that the $1999-2002$ sample was therefore $2 / 3$ rds of the entirety of the sample while the 2003-2004 sample was only 1/3rd of the entirety of the sample ranging from 1999 to 2004 (6-year cycle).

\section{Primary sampling units (PSU) and stratification variables}

The procedure for variance estimation (sampling errors) involved using PSU variable (SDMVPSU) and a strata variable (SDMVSTRA) (CDC, 2006). When NHANES files were released for the public beginning with NHANES 1999-2000, a new approach for the PSU variable was needed due to confidentiality and disclosure avoidance principles. Thus, beginning in the 1999-2000 cycle, Masked Variance Units (MVU's) 
were created which closely approximated the variances that would have been estimated if true variances estimated sampling errors (CDC, 2006). These MVU's and strata variables were also located on the demographic data files and could be incorporated in all two year cycles of data (CDC, 2006). Statistical software packages such as SUDAAN, STATA and SAS were necessary to handle the complex survey design which incorporated the PSU and strata variables necessary for estimating sampling errors (CDC, 2009).

\section{Data management and preparation}

Each dataset required for analysis from the three two-year NHANES surveys were downloaded from the public-use files from the NCHS website (http://www.cdc.gov/nchs/nhanes.htm). All variables included in the analyses were checked for consistency in content, procedural collection and code name. Any discrepancies were altered to assure similarity in the data.

The data were subsequently appended to form one large dataset within each twoyear cycle containing all needed variables. Frequency distributions were produced for all categorical variables and means, standard error of the means, and ranges were produced for all continuous variables to determine the extent of missing data, presence of outliers, and to check for normality of the data. Due to the suspicion of miscoding and aberrant behavior at the upper limits of the outcome variable of total water intake (TWI), the top $5 \%$ of TWI were trimmed from the data analysis (McKnight, McKnigh, Souraya, \& Figueredo, 2007). More specific information regarding the creation of the outcome variable of TWI is included in the section below (see "All Variables" section). 


\section{Missing data}

Missing data included answers left blank because the participant did not answer the question or did not participate in that aspect of the survey. Additionally, in the questionnaire section of the survey, if the participant answered, "don't know", this variable was redefined as missing. The NCHS rules for missing data were followed; these rules stated that a variable could be included in the analyses if it had less than $10 \%$ missing data (CDC, 2009). All variables that were missing or redefined as missing were excluded from the analyses. The variable for physical activity, which related to the amount of time spent doing a particular activity, was not selected due to the extensive amount of missing data. An alternative physical activity question was utilized instead (see "All Variables" section below).

\section{$\underline{\text { Eligibility criteria }}$}

The inclusion criteria were based on the ability to access a large representative sample of the US population in order to investigate the research questions of interest. The eligibility criteria were aimed at including as many adult participants as possible from the sample and only excluding those in whom the ability to maintain water balance adequately was questionable (e.g. pregnancy). The particular NHANES sample years were selected because of the specific data that were available during these years (CDC, 2010a; CDC, 2010b; CDC, 2010c).

Inclusion criteria for the study were: 1) non-pregnant individuals aged 19 and older; 2) participation in the continuous NHANES survey collected in the years 1999- 
2000, 2001-2002, and 2003-2004; and 3) participation in the 24 hour dietary recall with complete and reliable responses.

Exclusion criteria included individuals who had fasted during the 24 hour dietary recall. Individuals who had fasted were also excluded from similar studies using total water intake (Kant et al., 2009). Exclusion also included those participants who had a very active physical activity (PA) level, because elevated PA level is known to be associated with elevated water losses through sweat (Kant et al., 2009; IOM, 2005a). Additional exclusions included those participants who had self-reported congestive heart failure and were taking certain diuretics (see "All Variables" section for specific diuretics that were excluded). These exclusions were in accordance with other studies investigating diuretic usage, congestive heart failure, and fluid intake (Paterna, 2009; Paterna, 2008). Specific information regarding these eligibility variables are provided below under inclusion/exclusion criteria variables.

\section{$\underline{\text { Variables for determination of inclusion/exclusion criteria }}$}

Fasted individuals: This was a continuous variable expressed in kcals based on the 24 hour dietary recall variable of total kcals (DR1TKCAL) from the MEC examination portion for all individuals over age 19 years. Any individual with a total dr1tkcal of zero (i.e. fasting) was excluded from this study.

Self-report of congestive heart failure: This was a categorical variable defined as 1) yes and 2) no, based on the question "has a doctor or other health professional ever told you that you have congestive heart failure?" Participants who have congestive heart 
failure are likely to retain fluids and have an abnormal positive water balance (Paterna, 2009).

Names of diuretics: This was a nominal variable named for the prescription medication taken by the participant. Each medication was listed under the variable NHCODE as numbers which were associated to a specific diuretic. All reported drug names were converted to a standard generic drug name for data release. The diuretics excluded were those generic and brand diuretics including thiazide, potassium sparing, and loop diuretics. The specific diuretics and the associated code names that were excluded from the current study were chlorothiazide (12400), chlorothalidone (13800 and 13900), hydrochlorothiazide (29600, 29400, 29500, 29700, 29800, 29900, 30000, 30100, 30200, 30300, 30400, 90800, 90900, and 91000), indapamide (32500), methyclothiazid (39200 and 39300), bumetanide (08900), furosemide (26800), torsemide (57500), spironolactone (53800), and triamterene (58400). The variables 13900, 29400, 29500, $30000,39200,39300$ were not included in the 2001-2002 dataset. In 2001-2002 the variables for hydrochlorothiazide added were 90800, 90900, and 91000 and methychlothiazid was taken off of the thiazide diuretic list without explanation. Diuretics that were excluded cause negative fluid balance to occur by increasing urination in an attempt to rid the body of excess fluid (Ingham \& Byard, 2009). Since diuretic usage has been shown in the literature to affect water balance (Wakefield et al., 2009), participants with these specific self-reported diuretic usage were excluded.

Self-report of physical activity level: This was a categorical variable based on responses to the question "Please tell me which of these four sentences best describes your usual daily activities? These may include your work, housework if you are a 
homemaker, going to and attending classes if you are a student, and what you normally do throughout a typical day if you are a retiree or unemployed." The answer options were: 1) you sit during the day and do not walk about very much, 2) you stand or walk about a lot during the day, but do not have to carry or lift things very often, 3) you lift light loads or have to climb stairs or hills often, and 4) you do heavy work or carry heavy loads. Participants who responded with number four were excluded since water losses through sweating could affect water balance (Guyton \& Hall, 2006; IOM, 2005a) [Note: Although NHANES has other questions related to PA, this was the question that included usual activity of participants while the other questions addressed PA that may occur at any time over the last 30 days, making it less likely that the participant engaged in that activity on the day of the 24-hour dietary recall].

\section{Outcome variable}

Total water intake: Total water intake (TWI) was a created (named TOTGWATR) continuous variable expressed in $\mathrm{ml}$ (converted from grams to $\mathrm{ml}$ as 1gram of water $=1 \mathrm{ml}$ of water) by the sum of several variables from the datasets. The variables that were added together were the total grams of moisture from food and nonwater beverages (DR1TMOIS), the grams of total plain drinking water (DR1_320) from fountain, tap, spring, unsweetened-carbonated (DR1CWATR), and bottled water, and the total grams of water produced by metabolic water (another created variable).

The metabolic water was calculated using the principles of stoichiometry from general chemistry. Thus the metabolic water produced from 1 gram each of carbohydrates (DR1TCARB), protein (DR1TPROT), fat (DR1TTFAT), and alcohol 
(DR1TALCO) was 0.6, 0.4, 1.0, and 1.2 grams of water, respectively (Sheng, 2006). The inclusion of metabolic water in the total water variable is consistent with previous studies utilizing total water intake (Bossingham et al., 2005). The created variable of total water intake is consistent with previous studies utilizing NHANES data (Kant et al., 2009; Zizza et al., 2009).

\section{$\underline{\text { Potential predictor variables }}$}

Weight-based equations are two continuous variables expressed in $\mathrm{ml}$. These equations have been considered by others in the literature (see below for specific variable description). Both of these equations contained only the variable of $\mathrm{kg}$ body weight in the equation.

Linear equation (Chernoff, 1994b) applied a constant of 30 as the multiplier:

$30 \mathrm{ml} / \mathrm{kg}$ body weight (with a minimum of $1500 \mathrm{ml}$ )

Adjusted equation (Holliday and Segar, 1957) applied a constant of 20 as the multiplier after subtracting $20 \mathrm{~kg}$ from body weight and then added 1500 to the result:

$20 \mathrm{ml}^{*}(\mathrm{~kg}$ body weight -20$)+1500 \mathrm{ml}$

Energy-based equations are three continuous variables expressed in ml. All of these equations were based on the same basic principle of $1 \mathrm{ml}$ water per kcal energy expended and included the variables age, height, weight, and gender (see below for specific variable description) in the equation. The kcal expended portion of the equation was determined using three energy expenditure equations. These energy expenditure 
equations were consistent with the literature (Frankenfield et al., 2005; Gaillard et al., 2007; Spears, 2009).

Harris equation (Harris \& Benedict, 1919) contained different coefficients and a different y intercept than the other energy-based equations.

Females: $655+9.6$ weight $(\mathrm{kg})+1.85$ height $(\mathrm{cm})-4.7$ age $(\mathrm{yrs})$

Males: $66+13.8$ weight $(\mathrm{kg})+5$ height $(\mathrm{cm})-6.8$ age $(\mathrm{yrs})$

Mifflin equation (Mifflin et al., 1990) contained the same coefficients for both genders but contained different y intercepts between genders. This equation also contained different coefficients and a different y intercept than the other energy-based equations.

Female: $-161+9.99$ Weight $(\mathrm{kg})+6.25$ Height $(\mathrm{cm})-4.92$ Age $(\mathrm{yrs})$

Male: $5+9.99$ Weight $(\mathrm{kg})+6.25$ Height $(\mathrm{cm})-4.92$ Age $(\mathrm{yrs})$

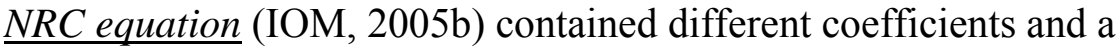
different $y$ intercept than the other energy-based equations and contained one extra variable of physical activity (described below).

Female: $354-6.91$ Age (yrs) + 9.36 Weight $(\mathrm{kg}) *$ PA +726 Height $(\mathrm{m}) *$ PA

Male: 662 - 9.53 Age (yrs) + 15.91 Weight (kg) * PA + 540 Height (m) * PA

Other predictors and potential confounding variables

Gender was a categorical variable defined as 1) male, 2) female (reference group). This variable was considered an important variable in all of the specific aims. 
Race/ethnicity was a categorical variable defined as 1) Mexican American, 2) Other Hispanic, 3) Non-Hispanic White, 4) Non-Hispanic Black, and 5) Other race including multiracial (reference group). This variable was incorporated in the model building in specific aim 4.

Age: was a continuous variable expressed in years.

Fat-free mass: was a continuous variable expressed in $\mathrm{kg}$ (BIDFFM) and was measured using bioelectrical impedance analysis (BIA). Bioelectrical impedance analysis was performed on participants aged 19-49 years using a multi-frequency bio-impedance spectroscopy (BIS). Bio-impedance spectroscopy data were collected with a HYDRA ECF/ICF Bio-Impedance Spectrum Analyzer (Model 4200) manufactured by Xitron Technologies, Inc., San Diego, California. Although a 6-hour fast was recommended by Xitron Technologies for the best BIA results and although there was a record kept for all participants who had fasted for laboratory testing, a snack was provided after the laboratory testing. Documentation of the time frame between the provision of a snack and the consumption of a snack was not available. Therefore, the assumption for this variable was that all participants were properly hydrated and not fasted. The BIS value provided a measure of the fat-free mass as has been used similarly in the literature (Han et al., 2010; Utter et al., 2010).

Body mass index was a calculated variable expressed in $\mathrm{kg} / \mathrm{m}^{2}$. This variable was redefined into a categorical variable for specific aim 3 as 1) BMI $<18.5,2$ ) BMI 18.524.9, 3) BMI 25-29.9, and 4) $\mathrm{BMI} \geq 30$. This variable was also considered as a continuous variable in specific aim 4. 
Height was a measured continuous variable expressed in cm except for determining BMI. When BMI was calculated, height was expressed in meters. Weight was a measured continuous variable expressed in $\mathrm{kg}$.

Total Kcal was a continuous variable representing the total kcal ingested in the diet in the previous twenty-four hours as determined by the 24-hour dietary recall.

Carbohydrate was a continuous variable expressed in grams representing the total amount of carbohydrate ingested in the diet in the previous twenty-four hours as determined by the 24-hour dietary recall.

Protein was a continuous variable expressed in grams representing the total amount of protein ingested in the diet in the previous twenty-four hours as determined by the 24-hour dietary recall.

$\underline{\text { Fat }}$ was a continuous variable expressed in grams representing the total amount of fat ingested in the diet in the previous twenty-four hours as determined by the 24-hour dietary recall.

Alcohol was a continuous variable expressed in grams representing the total amount of alcohol ingested in the diet in the previous twenty-four hours as determined by the 24-hour dietary recall.

\section{Metabolic Water production from:}

Carbohydrate was a continuous variable expressed in $\mathrm{ml}$ representing the total amount of water produced by ingestion of carbohydrates. One gram of carbohydrates yields $0.6 \mathrm{ml}$ of metabolic water (Sheng, 2006). 
Protein was a continuous variable expressed in $\mathrm{ml}$ representing the total amount of water produced by ingestion of protein. One gram of protein yields $0.4 \mathrm{ml}$ of metabolic water (Sheng, 2006).

Fat was a continuous variable expressed in $\mathrm{ml}$ representing the total amount of water produced by ingestion of fat. One gram of fat yields $1 \mathrm{ml}$ of metabolic water (Sheng, 2006).

Alcohol was a continuous variable expressed in $\mathrm{ml}$ representing the total amount of water produced by ingestion of alcohol. One gram of alcohol yields $1.2 \mathrm{ml}$ of metabolic water (Sheng, 2006).

Total metabolic water is a continuous variable expressed in $\mathrm{ml}$ representing the sum of metabolic water produced by carbohydrates, protein, fat, and alcohol.

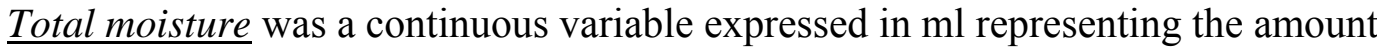
of water in all food and beverages ingested in the diet in the previous twenty-four hours from the 24-hour dietary recall.

Total plain drinking water is a continuous variable expressed in $\mathrm{ml}$ representing the amount of water ingested in the diet from tap, spring, fountain, and bottled water in the previous twenty-four hours from the 24-hour dietary recall.

Total carbonated water is a continuous variable expressed in $\mathrm{ml}$ representing the amount of water ingested in the diet from unsweetened carbonated water in the previous twenty-four hours from the 24-hour dietary recall.

Osmolality was a measured continuous variable expressed in $\mathrm{mOsm} / \mathrm{kg}$ and represented the amount of sodium, potassium, blood urea nitrogen and glucose in the 
blood. This variable was determined from blood collected after the participants had fasted for a minimum of six hours.

\section{$\underline{\text { Statistical analyses }}$}

All analyses (descriptive and inferential) were performed using weighted statistics appropriate for a multi-stage complex survey sampling design. Descriptive statistics reported for continuous variables were sample size, means, standard errors, and ranges. The continuous variables reported were age, carbohydrate, protein, fat, alcohol, weight, height, BMI, FFM, total kcals, osmolality, metabolic water from protein, metabolic water from carbohydrate, metabolic water from fat, metabolic water from alcohol, total metabolic water, total grams of moisture, total plain drinking water, total unsweetened carbonated water, total water intake, Linear equation, Adjusted equation, Harris equation, Mifflin equation, and NRC equation. Descriptive statistics reported for categorical variables were sample size and frequencies. The categorical variables reported were gender, race/ethnicity, BMI categories, and physical activity level.

All analyses were conducted using SAS 9.2 statistical software (SAS Institute, Cary, NC, USA). The SAS software was selected because of its capability to adjust for the complex multi-stage probability sample survey design of NHANES. The probability of committing a type 1 error was set at $5 \%($ alpha $=0.05)$ with statistical significance reached when observed $\mathrm{p}$ value was less than 5 percent. In Specific Aim 1, ten comparisons were performed on the same dataset. In order to avoid making a type 1 error due to multiple comparisons, alpha was set in accordance with the Hochberg Adjustment for multiple comparisons (Benjamini \& Hochberg, 1995). Therefore, the alpha of 0.05 
was divided by the 10 comparisons and a new alpha of 0.005 was set for comparison in this aim. Likewise in Specific Aim 2 where there were five multiple comparisons a new alpha of 0.01 was set (Benjamini \& Hochberg, 1995). A summary of the SAS commands in the analyses are displayed in Table 1.

\section{$\underline{\text { Inferential analysis and assumptions }}$}

Outliers in a dataset have the potential to distort results of an inferential analysis. The dataset was screened for missing data and inferential analysis was performed to check the assumptions for these statistical tests including the absence of outliers, normality, linearity and homoscedasticity as relates to eight continuous variable constructs of (a) total water intake (TOTGWATR), (b) BMI (BMXBMI), (c) Estimated fat-free mass (BIDFFM), (d) Linear equation (LINWGT), (e) Adjusted equation (ADJWGT), (f) Harris equation (HARRIS), (g) Mifflin equation (MIFFLIN), and (h) NRC equation (NRC).

A visual check of the boxplots for the continuous variable constructs in the analysis indicated no outliers on the TOTGWATR variable. Outliers were indicated on the other seven variables. However, the outlier values were within acceptable ranges because if the other assumptions are met regression is robust to violations of normality and to outliers (Tabachnick \& Fidell, 2007). And a check of the means of all variables versus the 5\% trimmed means indicated only small differences before and after trimming the outcome variable. A few of the variables returned extremely high values as indicated in Table 3. For example the maximum of kcals was 9,120. Although this was a high value, it was physiologically possible for individuals to consume this many kcals. 
Excluding data that may be feasible is not recommended while preserving the maximal amount of data is desirable (Kleinbaum, 2008). Therefore it was determined that outliers including kcals were not impacting the dataset by inflating the means and that the outlier assumption was not violated.

Normality for the eight continuous variables was investigated via the Kolmogorov-Smirnov and Anderson Darling tests for normality. Normality was not indicated for any of the eight inferential analysis variables. But, sample size can affect the goodness of fit tests that were performed and may return significant deviations from normality when none is apparent. A visual check of histograms and Normal QQ plots of the distributions for the eight variable constructs indicated reasonably normal shaped distributions with some right skew. However, none of the variables had a skewness value of $>1.5$, which is an acceptable level of skewness (Kleinbaum, 2008).

The outliers causing the right skew were not impacting the dataset. Correlation and regression analysis are robust to deviations from normality when the sample sizes are large and when other assumptions are met (Tabachnick \& Fidell, 2007) as they were in this study. Logarithmic and root transformations of the variables did not return improved distributions. It should also be noted that transformations would cause difficulty in interpretation of analysis results. It was therefore determined that the normality assumption was not seriously violated for the reasons stated above and that the data would be analyzed with the $5 \%$ trimmed outcome variable but without further transformations. 
Assumptions of linearity between study variables and homoscedasticity, requirements for correlational analysis, were checked with scatterplots of the data. The assumptions of linearity and homoscedasticity were not violated.

Multicollinearity diagnostics for multiple regression analyses were performed. During multiple regression analyses involving all predictors, multicollinearity was noted for control predictor variables of height, weight, and BMI using the variance inflation factor (VIF) for the data. The collinear predictors were sequentially removed to create the final regression model. Diagnostics on the final regression model did not indicate multicollinearity of the predictors and therefore the assumption of absence of multicollinearity was not violated. 
Table 1. List of commands used in SAS version 9.2

\begin{tabular}{ll}
\hline SAS Command & Purpose \\
\hline sddsrvyr & $\begin{array}{l}\text { Distinguishes which survey years were in the analysis } \\
\text { For example: if year=1, then survey years where 1999- } \\
2000\end{array}$ \\
\hline proc surveyfreq & Frequency for survey data \\
\hline tables & Cross-tabulations \\
\hline proc surveymean & Means, medians, range etc for survey data \\
\hline proc univariate & $\begin{array}{l}\text { Check normality and assumptions of analyses in } \\
\text { unweighted data }\end{array}$ \\
\hline var & $\begin{array}{l}\text { Variable statement for specifying variables on which to do } \\
\text { analyses }\end{array}$ \\
\hline proc surveyreg & Regression procedure for survey data \\
\hline sdmvpsu & Cluster variable in survey analysis of PSU \\
\hline sdmvstra & Strata variable in survey analysis \\
\hline weight & $\begin{array}{l}\text { Weight variable created in survey analysis } \\
\text { For example: WTMEC6YR is the weight variable used } \\
\text { for participants who were examined in the MEC }\end{array}$ \\
\hline domain & $\begin{array}{l}\text { For subpopulation analysis in survey data and to account } \\
\text { for the sampling weight differences due to the exclusion } \\
\text { of specific segments of the population }\end{array}$ \\
\hline class & For categorical variable analysis \\
\hline VIF & $\begin{array}{l}\text { To check for multicollinearity. The VIF was calculated } \\
\text { from the weighted } r^{2} \text { of each potential predictor variable. } \\
\text { The surveyreg procedure was performed using the rest of } \\
\text { the predictors as independent variables. All } r^{2} \text { values were } \\
\left.\text { recorded. VIF is then 1/(1 }-r^{2}\right) .\end{array}$ \\
&
\end{tabular}

Specific aim 1

To determine agreement among existing equations used to estimate water requirements of adults using the continuous NHANES Survey 1999-2004 dataset. Sample size

From a similar study which compared four different body composition methods to one another (six comparisons), the author was able to show significance at an alpha of 0.05 with only 15 subjects in total (Fuller, 1994). In this specific aim, five different 
equations were compared to each other (ten comparisons between equations). The NHANES 1999-2004 data provided more than adequate sample size to show significant differences between equations.

Analyses for specific aim 1

The criteria for agreement were twofold: 1) if the pair of variables demonstrated a strong $(r>0.7)$ or medium $(0.4<r<0.7)$ degree of association (Overholser \& Sowinski, 2008), and 2) if the regression line produced by the pair of variables was not significantly different from the line of equality with the CI of the slope and intercept including one and zero, respectively (Volaufova, 2005). Criteria two would only be performed on those variables meeting criteria one; they demonstrated a strong $(r>0.7)$ or medium $(0.4<r<$ 0.7) degree of association.

In criteria one, simple linear regression models performed on each pair of variables for the five equations produced ten regression equations. The amount of shared variance found for each of the comparisons was detected using $r^{2}$ and the associated $p$ value. A correlation coefficient matrix compared Pearson's correlation and the associated $p$-value to determine the degree of association between each pair of equations (Overholser \& Sowinski, 2008). If the pair of variables demonstrated a large shared variance and a strong or medium degree of association as described above, criteria one would be satisfied.

In criteria two, linear regression analysis was performed to determine the agreement between variables. Each of the pairs of variables were regressed on one another and the $95 \%$ confidence interval (CI) was found for the slope and the intercept. For criteria two to be met, the regression line could not be significantly different from the 
line of equality, i.e. CI of the slope and intercept had to include one and zero, respectively (Volaufova, 2005). If the pair of variables demonstrated a weak degree of association ( $r$ $<0.3$ ), this would not meet the criteria for agreement and further testing would not occur (Overholser \& Sowinski, 2008). In this case the two variables would not be in agreement with one another.

The line of equality was defined as the line that would result if all values of one equation completely matched all values of the other equation $(x=y)$. This line of equality would be plotted beside the regression line. The line of equality contained a 45 degree angle from a point of $y=0$ and $x=0$. The line of equality also contained a slope of one and an intercept of zero. If the regression of one equation on the other equation agreed with the line of equality, it would not be significantly different from this line. However if the $95 \% \mathrm{CI}$ of the slope and intercept did not include the possibility that the slope of the regression of one variable on the other variable contained one and the intercept contained zero the two equations were not considered to be in agreement (Volaufova, 2005). Other investigators also incorporated this type of agreement analysis in their investigations (Claros et al., 2005; Huang et al., 2002; Huang et al., 2003).

The Hochberg Adjustment procedure was selected to correct for the problem of multiple comparisons (Benjamini \& Hochberg, 1995). Since there were ten comparisons in this analysis, the alpha level was set at 0.005 for this specific aim. 
The following depicts how each equation was paired to all of the other equations:

$\begin{array}{ccc}\text { Comparisons } & \text { Equation } & \text { Equation } \\ 1 & \text { Linear } & \text { Adjusted } \\ 2 & \text { Linear } & \text { Harris } \\ 3 & \text { Linear } & \text { Mifflin } \\ 4 & \text { Linear } & \text { NRC } \\ 5 & \text { Adjusted } & \text { Harris } \\ 6 & \text { Adjusted } & \text { Mifflin } \\ 7 & \text { Adjusted } & \text { NRC } \\ 8 & \text { Harris } & \text { Mifflin } \\ 9 & \text { Harris } & \text { NRC } \\ 10 & \text { Mifflin } & \text { NRC }\end{array}$

Specific aim 2

To determine agreement between existing equations used to estimate water requirements of adults and total water intake of adults using the continuous NHANES 1999-2004 dataset.

Sample size

From similar studies which compared fluid intake in institutionalized elderly subjects to weight-based and energy-based equations, authors were able to show significant results with 121 subjects (Holben et al., 1999; Chidester \& Spangler, 1997). Therefore, the NHANES 1999-2004 dataset provided more than adequate sample size to show significant differences between EWR equations and TWI. 
Analyses for specific aim 2

The criteria for agreement were twofold: 1) if the predictor and outcome variables demonstrated a strong $(r>0.7)$ or medium $(0.4<r<0.7)$ degree of association (Overholser \& Sowinski, 2008), and 2) if the regression line produced by the pair of variables was not significantly different from the line of equality with the $\mathrm{CI}$ of the slope and intercept including one and zero, respectively (Volaufova, 2005). Criteria two would only be performed on those variables meeting criteria one; they demonstrated a strong $(r$ $>0.7)$ or medium $(0.4<r<0.7)$ degree of association.

In criteria one, simple linear regression models performed between TWI (outcome variable) and each of the five EWR equations produced five regression equations. The amount of shared variance found for each of the comparisons was detected using $r^{2}$ and the associated $p$-value. A correlation coefficient matrix compared Pearson's correlation and the associated $p$-value to determine the degree of association between each pair of variables (Overholser \& Sowinski, 2008). If the pair of variables demonstrated a large shared variance and a strong or medium degree of association as described above, criteria one would be satisfied. If the pair of variables demonstrated a weak degree of association $(r<0.3)$, this would not meet the criteria for agreement and further testing would not occur (Overholser \& Sowinski, 2008).

In criteria two, TWI was regressed on each of the five EWR equations and the $95 \%$ confidence interval (CI) was found for the slope and the intercept. For criteria two to be met, the regression line could not be significantly different from the line of equality, i.e. CI of the slope and intercept had to include one and zero, respectively (Volaufova, 2005). Both conditions had to be satisfied to meet the criteria of agreement. Other 
investigators also incorporated this type of agreement analysis in their investigations (Claros et al., 2005; Huang et al., 2002; Huang et al., 2003).

The Hochberg Adjustment procedure was selected to correct for the problem of multiple comparisons (Benjamini \& Hochberg, 1995). Since there were five comparisons in this analysis, the alpha level was set at 0.01 for this specific aim.

Specific aim 3

To determine the correlation between fat-free mass (FFM) and total water intake using the continuous NHANES 1999-2004 dataset across the BMI range.

Sample size

Investigators in a similar study, which compared total body water measurements using an isotope dilution method, skinfold thickness, BIA, and flowing afterglow-mass spectrometry, were able to show significant results with 24 subjects (Smith, Engel, Diskin, Spanel, \& Davies, 2002). The NHANES 1999-2004 dataset expected to provide more than adequate sample size to show significant correlations between FFM and TWI in the four BMI categories.

Analysis for specific aim 3

Body mass index was divided into 4 categories for this specific aim. The four categories were (1) $<18.5$, (2) $18.5-24.9$, (3) $25-29.9$, and (4) $\geq 30$. Simple regressions were performed for the predictor variable, the estimated FFM (BIDFFM), and the outcome variable of total water intake (TWI), for each of the four BMI groups using the SAS proc surveyreg command. Square roots of the simple regression $r$-square statistics were taken to derive a Pearson's linear correlation coefficient in order to define 
the association between BMI and TWI for each of the four BMI group regressions. Correlation coefficients of $<0.30$ were considered weak associations. Correlation coefficients of 0.4 - 0.7 were considered medium associations. Correlation coefficients $>0.7$ were considered strong associations (Overholser \& Sowinski, 2008).

\section{Specific aim 4}

To derive and validate one or more practical equations for predicting total water intake using the continuous NHANES 1999-2004 datasets.

\section{Sample size}

Similar studies have shown that an equation can be derived and validated using 96-156 subjects in total (Huang et al., 2002, Huang et al., 2003). Due to the large sample size of NHANES, there was more than enough power to test specific aim 4.

\section{Model criteria and strategy}

For this specific aim the goal was to build and test one equation which at the outset contained FFM (model A) as a predictor of TWI. Additionally another equation which did not initially contain FFM (model B) as a predictor was expected to be derived and tested. The other predictor variables included in model A and B were selected based on the literature and their known relationships with water requirements and water intake (Kant et al., 2009; Zizza, et al., 2009). The variables considered for inclusion were race/ethnicity, kcals consumed, age, height, weight, gender, BMI, and osmolality.

Construction of the best regression models for A and B was accomplished using a set of criteria established prior to model construction (Kleinbaum, 2008). The first step was the selection of the maximum model by the deletion of predictors to be dropped 
based on preset criteria (Kleinbaum, 2008). This type of procedure involved the regression of the full model followed by the manual elimination of variables. Manual elimination of variables was necessary since the SAS surveyreg command did not contain the capabilities of automatic removal of variables. Therefore, a strict a priori protocol for variable removal was determined. These steps were followed for the development of final model A and final model B.

This method was chosen in order to decrease the possibility of making a type 1 error while allowing for the consideration of all possible basic predictors. The end goal of this method was to predict the most practical and parsimonious model which included clinically relevant variables supported by the literature (Kleinbaum, 2008).

The next step was to determine how the variables would be selected for inclusion in either of the models (model A or B). Each variable determined to be clinically significant in the literature was tested using univariate analysis. A p-value of 0.2 was considered to be an acceptable cut-off in the univariate analysis for each variable tested (Kleinbaum, 2008). Although higher than the usual $p$-value of 0.05 , the liberalization for this criterion was necessary in order to assure that all variables that were clinically significant were included despite not being statistically significant at the $5 \%$ level.

Appropriate final models A and B were to be selected by using manual backward elimination for each. Manual backward elimination started with a full regression of all clinically significant variables in model A and B. The variable with the highest $p$ value and lowest clinical significance according to the literature was sequentially eliminated from the subsequent regression model. This step by step backward elimination occurred until a final model was produced in model A and in model B. The regression with the 
highest $r^{2}$, highest $F$ statistic, lowest root mean square error (RMSE), and all significant variables was considered as the best fit and final model.

All regressions produced were checked for multicollinearity. The variance inflation factor (VIF) was calculated from the weighted $r^{2}$ of each potential predictor variable. The surveyreg procedure was performed using the rest of the predictors as independent variables. All $r^{2}$ values were recorded. The VIF was then calculated as 1/(1 $r^{2}$ ). The criterion to deal with multicollinearity was that the variable with the highest VIF and $>10$ would be sequentially removed from the regression model A and model B (Cody \& Smith, 2006). During the creation of final models A and B the regression was re-run with each sequential removal of a variable containing a VIF $>10$. When all variables that had a VIF $>10$ were removed, backward removal of variables with the highest $p$-value would commence. The variable with the highest $p$-value would be removed first and the regression re-run in a sequential manner. After the construction of each model the VIF was rechecked to assure multicollinearity was not a problem.

Models A and B were also checked for confounding variables. The criterion to deal with confounding variables was to calculate changes in the coefficients of the predictors for the methodological removal of a given variable one by one (Kleinbaum, 2008). First the coefficients and $p$-values for the full models were noted. With each subsequent removal of a variable that did not meet the variable criteria to remain in the model, the coefficient and $p$-values of the remaining variables were rechecked to determine any changes. A change of $20-25 \%$ in the coefficients between regressions was considered to be meaningful (Kleinbaum, 2008). Changes of $>25 \%$ for coefficients would imply confounding was a problem. 
Other considerations for model building were to center the variables, take the logarithm or the square root of the outcome variable. The logarithmic transformation would aid in meeting the normality assumption. The square root transformation of the dependent variable would be utilized to stabilize the variability or solve any problems of deviations from normality. Centering of the independent variables would solve the problem of multicollinearity. All of these data transformations were attempted in models $\mathrm{A}$ and $\mathrm{B}$ as well.

After checking the results of centering and of the logarithmic and square root transformation and observing that there was no change in the normality or variance stability of the data, it was decided that no transformations were to be completed in the final models A and B. The difficulty associated with interpretation of the final model and the desire for one or more practical equations to be developed were instrumental in this decision. Additionally, the data approached normality when the top 5\% of the TWI variable were trimmed. 


\section{CHAPTER IV.}

\section{RESULTS}

\section{$\underline{\text { Overview }}$}

This quantitative descriptive study researched four objectives as related to estimated water requirements (EWR) and total water intake (TWI). The four objectives were to: 1) Compare and determine agreement among currently used EWR equations; 2) Compare and determine agreement between currently used EWR equations and TWI; 3) Determine whether fat-free mass (FFM) is associated with TWI; and 4) Create and validate one or more practical EWR equations.

The results chapter was divided into three sections (a) sample characteristics and demographic findings; (b) investigation of assumptions as relates to inferential analysis; and (c) inferential analysis as relates to the four specific aims of this study. The chapter also contains a summary of results following each specific aim.

\section{$\underline{\text { Sample characteristics and demographic findings }}$}

The frequencies and weighted percentages for the categorical variables utilized in this study are presented in Table 2 . The weighted measures of central tendency for the continuous measures analyzed in this study are presented in Table 3. 
Table 2. Frequencies and percentages of categorical variables

\begin{tabular}{llr}
\hline Variable & Frequency & Percent \\
\hline Gender & & \\
$\quad$ Male & 5,041 & 44.8 \\
Female & 5,691 & 55.3 \\
& & \\
Race/ethnicity & 2,526 & 7.1 \\
$\quad$ Mexican American & 497 & 6.0 \\
$\quad$ Other Hispanic & 5,177 & 71.5 \\
$\quad$ Non-Hispanic White & 2,154 & 10.8 \\
$\quad$ Non-Hispanic Black & 378 & 4.6 \\
Multiracial/other & & \\
& & \\
Average level of physical activity each day & 26.2 \\
Sit during the day and do not walk around very much & 2,776 \\
Stand or walk about a lot during the day, but do not & & \\
have to carry or lift things very often & 6,111 & 54.5 \\
Lift light loads or have to climb stairs or hills often & 1,845 & 19.3 \\
& & \\
Body mass index (BMI) category & & \\
$\quad<18.5$ & 472 & 4.4 \\
18.5 - 24.9 & 3,381 & 31.5 \\
25 - 29.9 & 3,611 & 33.7 \\
$\geq 30$ & 3,133 & 29.2 \\
Missing & 135 & 1.3 \\
\hline
\end{tabular}

Note: Weighted percentages computed; $\mathrm{n}=10,732$ 
Table 3. Measures of central tendency for continuous variables

\begin{tabular}{|c|c|c|c|c|c|}
\hline \multirow[b]{2}{*}{ Variable } & \multirow[b]{2}{*}{$n$} & \multirow[b]{2}{*}{$M$} & \multirow[b]{2}{*}{ SEM } & \multicolumn{2}{|c|}{ Range } \\
\hline & & & & Min & Max \\
\hline Age (yr) & 10,732 & 46 & 0.3 & 19 & 85 \\
\hline Energy (kcal) & 10,732 & 2,115 & 13.1 & 5 & 9,120 \\
\hline Protein (gm) & 10,732 & 79 & 0.7 & 0 & 718 \\
\hline Carbohydrate (gm) & 10,732 & 261 & 1.7 & 1 & 1,487 \\
\hline Total fat (gm) & 10,732 & 80 & 0.5 & 0 & 449 \\
\hline Alcohol (gm) & 10,732 & 9 & 0.4 & 0 & 552 \\
\hline Moisture (ml) & 10,732 & 1,924 & 18.7 & 2 & 5,900 \\
\hline Weight $(\mathrm{kg})$ & 10,546 & 79 & 0.3 & 26 & 209 \\
\hline Standing height $(\mathrm{cm})$ & 10,535 & 168 & 0.1 & 130 & 203 \\
\hline Body mass index $\left(\mathrm{kg} / \mathrm{m}^{2}\right)$ & 10,470 & 28 & 0.1 & 12 & 66 \\
\hline Estimated fat-free mass $(\mathrm{kg})$ & 4,480 & 52 & 0.3 & 24 & 106 \\
\hline Osmolality (mOsm/kg) & 10,147 & 278 & 0.2 & 241 & 310 \\
\hline Metabolic water from protein $(\mathrm{ml})$ & 10,732 & 32 & 0.3 & 0 & 287 \\
\hline Metabolic water from carbohydrate $(\mathrm{ml})$ & 10,732 & 156 & 1.0 & 0.71 & 892 \\
\hline Metabolic water from fat (ml) & 10,732 & 80 & 0.5 & 0 & 449 \\
\hline Metabolic water from alcohol (ml) & 10,732 & 11 & 0.5 & 0 & 662 \\
\hline Total metabolic water produced $(\mathrm{ml})$ & 10,732 & 280 & 1.8 & 0.83 & 1,270 \\
\hline Total plain drinking water $(\mathrm{ml})$ & 10,732 & 1,072 & 20.0 & 0 & 5,310 \\
\hline Total US carbonated water $(\mathrm{ml})$ & 7,431 & 11 & 2.4 & 0 & 2,575 \\
\hline Total water intake (TWI) (ml) & 10,732 & 3,283 & 24.6 & 236 & 6,284 \\
\hline Linear equation $(\mathrm{ml})$ & 10,546 & 2,374 & 9.4 & 1,500 & 6,273 \\
\hline Adjusted equation (ml) & 10,546 & 2,681 & 6.3 & 1,612 & 5,282 \\
\hline Harris equation (ml) & 10,470 & 1,614 & 4.1 & 823 & 3,693 \\
\hline
\end{tabular}




\begin{tabular}{lccccc} 
Variable & $n$ & $M$ & SEM & Min & Max \\
\hline Mifflin equation (ml) & 10,470 & 1,531 & 3.8 & 642 & 3,113 \\
NRC equation (ml) & 10,470 & 2,192 & 5.3 & 1,114 & 4,741
\end{tabular}

Note. $M=$ Mean; $S E M=$ Standard Error of Mean; Min = Minimum; Max = Maximum; mOsm $=$ Milliosmoles; US = unsweetened; Weighted mean and weighted standard error of the mean.

The cases included in the sample ranged in age from 19 to 85 years $(M=46$ years, $S E M=0.28$ years $)$. The sample contained fewer males $(5,041$ cases, $44.8 \%)$ than females $(5,691$ cases, $55.3 \%)$. The majority of the race/ethnicity of the sample was Non-Hispanic White $(71.5 \%)$ with the smallest group belonging to Multiracial or other race $(4.6 \%)$. The majority of the sample $(54.5 \%)$ reported their daily activity to be "Standing or walking about a lot during the day, but do not have to carry or lift things very often." The sample was evenly divided in terms of BMI for those in the categories of, $18.5-24.9(31.5 \%)$, $25-29.9(33.6 \%)$, and $\geq 30(29.2 \%)$. Only those in the underweight category of $<18.5$ had the smallest percentage of sample size (4.4\%). There was a large range of between 236 to $6,284 \mathrm{ml}$ in average total water consumption $(M=3,283 \mathrm{ml}$, SEM $24.6 \mathrm{ml})$. $\underline{\text { Results of specific aim } 1}$

Specific aim 1: To determine agreement among existing equations used to estimate water requirements of adults using the continuous NHANES Survey 1999-2004 datasets.

Hypothesis 1: Estimates of water requirements among existing weight-based and energy-based equations will lack agreement with each other using the continuous NHANES 1999-2004 datasets. 
The computations for each of the five EWR equations, as well as the TWI variable derived for this study are presented in Table 4. Simple regressions were performed for each bivariate pairing of the EWR equations using the SAS proc surveyreg command. 
Table 4. Computation Information for the five EWR equations

\begin{tabular}{|c|c|c|}
\hline $\begin{array}{l}\text { Type of } \\
\text { Equation }\end{array}$ & $\begin{array}{l}\text { Name of } \\
\text { Equation }\end{array}$ & Equation \\
\hline \multirow{2}{*}{$\begin{array}{l}\text { Weight- } \\
\text { Based }\end{array}$} & Linear & $30 \mathrm{ml} / \mathrm{kg}$ body weight (minimum $1500 \mathrm{ml}$ ) \\
\hline & Adjusted & $20 \mathrm{ml}^{*}(\mathrm{~kg}$ body weight -20$)+1500 \mathrm{ml}$ \\
\hline \multirow{3}{*}{$\begin{array}{l}\text { Energy- } \\
\text { Based } \\
\text { 1ml/kcal }\end{array}$} & Harris & $\begin{array}{l}\text { F: } 655+9.6 \text { weight }+1.85 \text { height }-4.7 \text { age } \\
\text { M: } 66+13.8 \text { weight }+5 \text { height }-6.8 \text { age }\end{array}$ \\
\hline & Mifflin & $\begin{array}{l}\text { F: }-161+9.99 \text { weight }+6.25 \text { height }-4.92 \text { age } \\
\text { M: } 5+9.99 \text { weight }+6.25 \text { height }-4.92 \text { age }\end{array}$ \\
\hline & $\mathrm{NRC}$ & $\begin{array}{l}\text { F: } 354-6.91 \text { age }+9.36 \text { weight } * \mathrm{PA}+726 \text { height } * \\
\text { PA } \\
\text { M: } 662-9.53 \text { age }+15.91 \text { weight } * \mathrm{PA}+540 \text { height } \\
* \text { PA }\end{array}$ \\
\hline $\begin{array}{l}\text { Derived for } \\
\text { Study }\end{array}$ & TWI & $\begin{array}{l}\text { total moisture from food }+ \text { total plain drinking water } \\
+ \text { total unsweetened carbonated water }+ \text { total } \\
\text { metabolic water }\end{array}$ \\
\hline
\end{tabular}

Note. Mifflin = Mifflin St. Jeor equation; Harris = Harris Benedict equation; NRC = National Research Council equation; PA = Physical activity. TWI = Total water intake variable constituents were computed in milliliters. Weight was measured in $\mathrm{kg}$; height was measured in cm except for NRC equation height measured in meters; age was measured in years; PA $=1$ in sedentary and low active adults. 
Table 5a. Simple linear regression to examine the $95 \% \mathrm{CI}$ of the slope

\begin{tabular}{cccccccc}
\multicolumn{2}{c}{ Variables } & & & \multicolumn{3}{c}{$95 \%$ CI of Slope } & \\
\cline { 1 - 1 } & Y & X & $n$ & Slope & Lower & Upper & $r^{2}$ \\
\cline { 1 - 1 } Linear & Adjusted & 10,546 & 1.485 & 1.482 & 1.488 & 0.998 \\
Linear & Harris & 10,470 & 1.629 & 1.602 & 1.657 & 0.756 \\
Linear & Mifflin & 10,470 & 1.711 & 1.673 & 1.748 & 0.735 \\
Linear & NRC & 10,470 & 1.039 & 1.014 & 1.063 & 0.592 \\
Adjusted & Harris & 10,470 & 1.096 & 1.078 & 1.114 & 0.756 \\
Adjusted & Mifflin & 10,470 & 1.153 & 1.128 & 1.178 & 0.737 \\
Adjusted & NRC & 10,470 & 0.699 & 0.683 & 0.715 & 0.593 \\
Harris & Mifflin & 10,470 & 1.049 & 1.041 & 1.058 & 0.971 \\
Harris & NRC & 10,470 & 0.705 & 0.670 & 0.709 & 0.956 \\
Mifflin & NRC & 10,470 & 0.661 & 0.658 & 0.664 & 0.954
\end{tabular}

Note: $\mathrm{Y}=\overline{\text { Dependent Variable (outcome); } \mathrm{X}=\text { Independent Variable (predictor) }}$; Slope $=$ Beta Unstandardized Coefficient; $\mathrm{CI}=$ Confidence Interval; Linear $=$ Linear equation; Adjusted $=$ Adjusted equation; Mifflin $=$ Mifflin St. Jeor equation; Harris $=$ Harris Benedict equation; $\mathrm{NRC}=$ National Research Council equation; TWI $=$ Total Water Intake. All model coefficients were statistically significant at $p<.0001$. 
Table 5b. Simple linear regression to examine the $95 \%$ CI of the intercept

\begin{tabular}{cccccccc}
\multicolumn{2}{c}{ Variables } & & & \multicolumn{3}{c}{$95 \%$ CI of Intercept } & \\
\cline { 1 - 1 } & Y & & & Intercept & Lower & Upper & $r^{2}$ \\
\cline { 1 - 1 } Linear & Adjusted & 10,546 & $-1,606.63$ & $-1,614.64$ & $-1,598.62$ & 0.998 \\
Linear & Harris & 10,470 & -255.86 & -298.52 & -213.19 & 0.756 \\
Linear & Mifflin & 10,470 & -245.28 & -300.74 & -189.81 & 0.735 \\
Linear & NRC & 10,470 & 97.91 & 46.22 & 149.60 & 0.592 \\
Adjusted & Harris & 10,470 & 910.98 & 882.47 & 939.49 & 0.756 \\
Adjusted & Mifflin & 10,470 & 915.73 & 878.95 & 952.51 & 0.737 \\
Adjusted & NRC & 10,470 & $11,48.31$ & $11,13.80$ & $1,182.82$ & 0.593 \\
Harris & Mifflin & 10,470 & 7.71 & -4.80 & 20.22 & 0.971 \\
Harris & NRC & 10,470 & 70.34 & 60.88 & 79.88 & 0.956 \\
Mifflin & NRC & 10,470 & 82.74 & 75.75 & 89.73 & 0.954
\end{tabular}

Note: $\overline{\mathrm{Y}=\text { Dependent Variable (outcome); } \mathrm{X}=\text { Independent Variable (predictor); } \mathrm{C}} \mathrm{I}=$ Confidence Interval; Linear $=$ Linear equation; Adjusted = Adjusted equation; Mifflin = Mifflin St. Jeor equation; Harris = Harris Benedict equation; NRC = National Research Council equation; TWI $=$ Total Water Intake. All model coefficients were statistically significant at $p<.0001$.

All regression models and model coefficients were statistically significant at an alpha of $0.005(p<0.0001)$. Due to the ten multiple comparisons the alpha was set at 0.005 .

The model $r^{2}$ for all regressions involving the five EWR equations were above 0.50 , meaning that over $50 \%$ of the variability in each of the estimated water intake 
equation model outcomes could be accounted for by the model predictors. The regression of the Linear equation on the predictor of the Adjusted equation returned the highest $r^{2}$ value $\left(r^{2}=.998\right)$. Thus, $99.8 \%$ of the model variance in the Adjusted equation was accounted for by the Linear equation. The regression of the Linear equation on the NRC equation returned the lowest $r^{2}$ value $\left(r^{2}=0.592\right)$, indicating that $59.2 \%$ of the model variance in the $\mathrm{NRC}$ equation was accounted for by the Linear equation.

The slopes and the $95 \% \mathrm{CI}$ of the slopes for each of the regression lines corresponding to each of the equation comparisons are presented in Table 5a. For example the Linear equation was regressed on the Adjusted equation (Figure 2). These two equations had a high correlation coefficient $(r=0.99, p<0.0001)$, meaning that higher values of one were associated with higher values of the other (Overholser \& Sowinski, 2008). However, in order for equations to be in agreement, the regression line formed when one equation is regressed on the other must not be significantly different from the line of equality. Agreement would result if the plotted regression line of the Linear equation against the Adjusted equation was close to the line of equality $(\mathrm{x}=\mathrm{y})$ (Figure 2). The line of equality contained a 45 degree angle from a point of $\mathrm{y}=0$ and $\mathrm{x}=$ 0 , with a slope of one and an intercept of zero. Since the $95 \%$ CI of the slope and intercept did not include the possibility that the slope of the regression of the Linear equation on the Adjusted equation contained one nor did the intercept contain zero, the Linear equation did not agree with the Adjusted equation.

The intercepts and the $95 \% \mathrm{CI}$ of the intercepts for each of the regression lines corresponding to each of the equation comparisons is presented in Table 5b. None of the equation comparisons with a statistically significant $r^{2}$ or with a high correlation 
coefficient returned a regression equation with the $95 \% \mathrm{CI}$ of the slope to include one and the $95 \%$ CI of the intercept to include zero. That is to say that all of the equation comparisons were significantly different from the line of equality and therefore none of these equations agreed with each other. A random sample graphic of scatterplots between the bivariate pairings of the EWR equations are displayed in Figures 2 through 11.

Table 6. Correlation coefficients of estimated water intake equation and total water intake equation*

\begin{tabular}{lccccc}
\hline Variable & 1 & 2 & 3 & 4 & 5 \\
\hline 1. Mifflin & 1.0 & & & & \\
2. Harris & $0.986^{*}$ & 1.0 & & & \\
3. NRC & $0.977^{*}$ & $0.978^{*}$ & 1.0 & & \\
4. Linear & $0.857^{*}$ & $0.869 *$ & $0.769 *$ & 1.0 & \\
5. Adjusted & $0.859 *$ & $0.869 *$ & $0.770 *$ & $0.999 *$ & 1.0 \\
6. TWI & 0.273 & 0.267 & 0.284 & 0.182 & 0.183
\end{tabular}

Note. Linear $=$ Linear equation; Adjusted = Adjusted equation; Mifflin = Mifflin St. Jeor equation; Harris = Harris Benedict equation; $\mathrm{NRC}=$ National Research Council equation; TWI $=$ Total Water Intake. All correlations statistically significant at the $p<.0001$ level. Correlation coefficient showed a strong $(r>0.07)$ degree of association in the model.

Results of the correlational analysis are presented in Table 6. Correlations between the bivariate estimated water intake equation outcomes were all positive and strong with correlation coefficients of over $0.70(p<0.0001)$. The Adjusted and Linear equations indicated the strongest association with a correlation coefficient of almost one $(r=0.998)$. Other correlations above .90 included Mifflin with Harris $(r=0.985)$, Mifflin with NRC $(r=0.977)$, and NRC with Harris $(r=0.978)$. 
Figure 2. Regression of the Linear and Adjusted equations compared to the line of equality

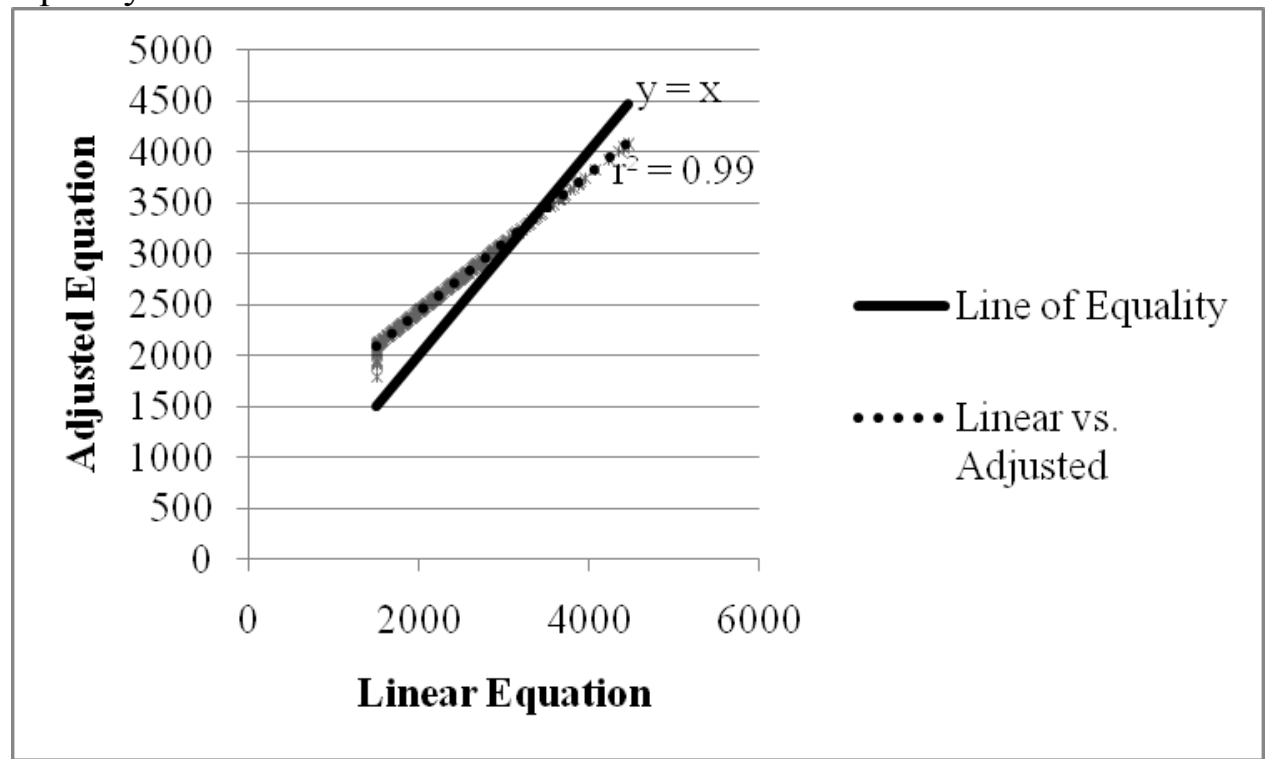

The regression of the Linear and Adjusted equations produced a strong correlation $(r=$ $0.999, p<0.0001)$. However, the criteria for agreement between the Linear and Adjusted equations were not met as the regression line was too different from the $\mathrm{y}=\mathrm{x}$ line of equality (i.e., 95\% CI of the slope did not include one and 95\% CI of the intercept did not include zero). 
Figure 3. Regression of the Linear and Harris equations compared to the line of equality

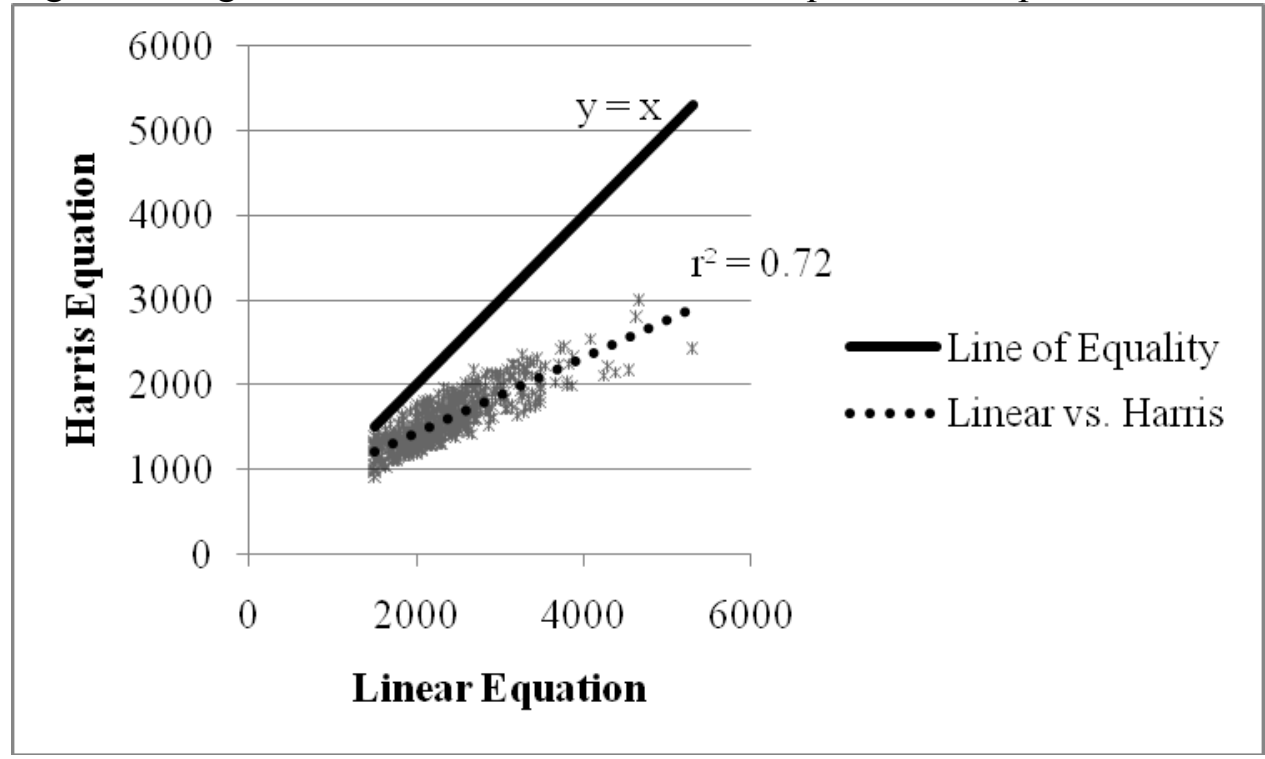

The regression of the Linear and Harris equations produced a strong correlation $(r=$ $0.869, p<0.0001$ ), but the criteria for agreement between the Linear and Harris equations were not met as the regression line was too different from the $\mathrm{y}=\mathrm{x}$ line of equality. 
Figure 4. Regression of the Linear and Mifflin equations compared to the line of equality

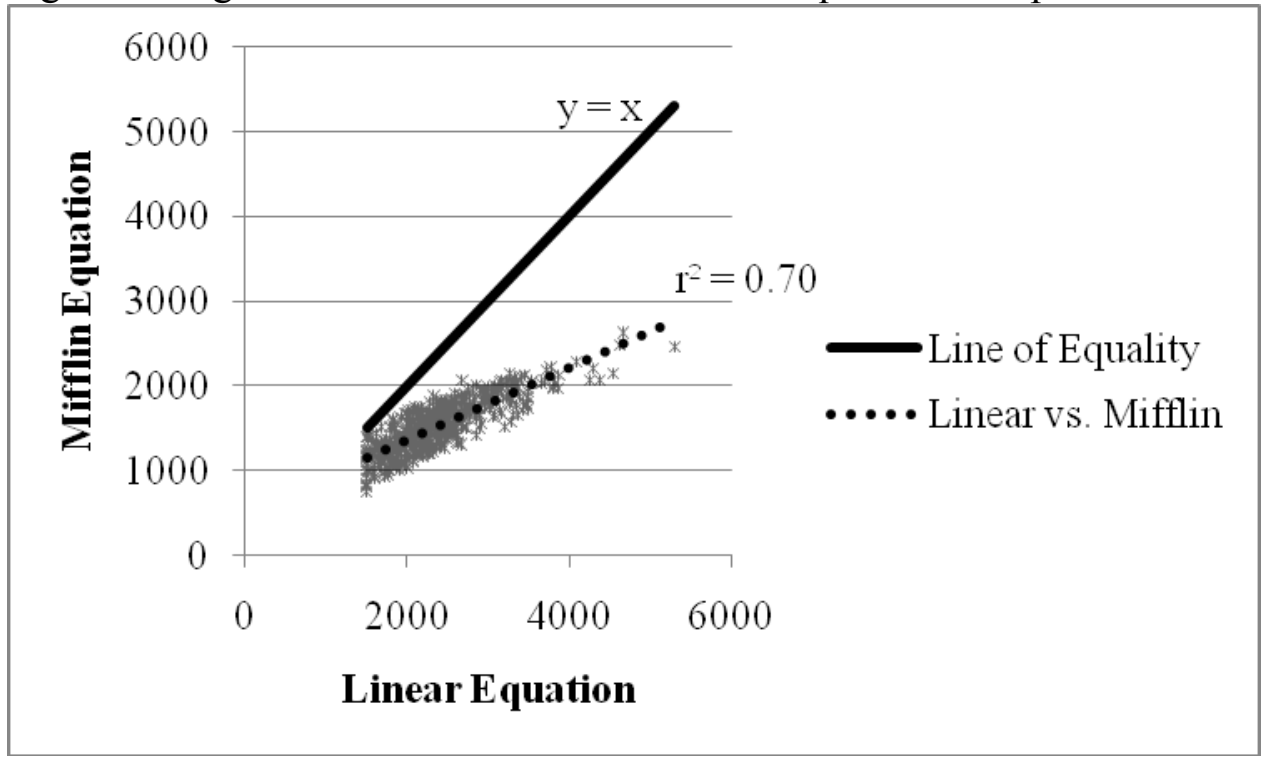

The regression of the Linear and Mifflin equations produced a strong correlation $(r=$ 0.857, $p<0.0001$ ), but the criteria for agreement between the Linear and Mifflin equations were not met as the $95 \%$ CI of the slope did not include one and $95 \%$ CI of the intercept did not include zero. 
Figure 5. Regression of the Linear and NRC equations compared to the line of equality

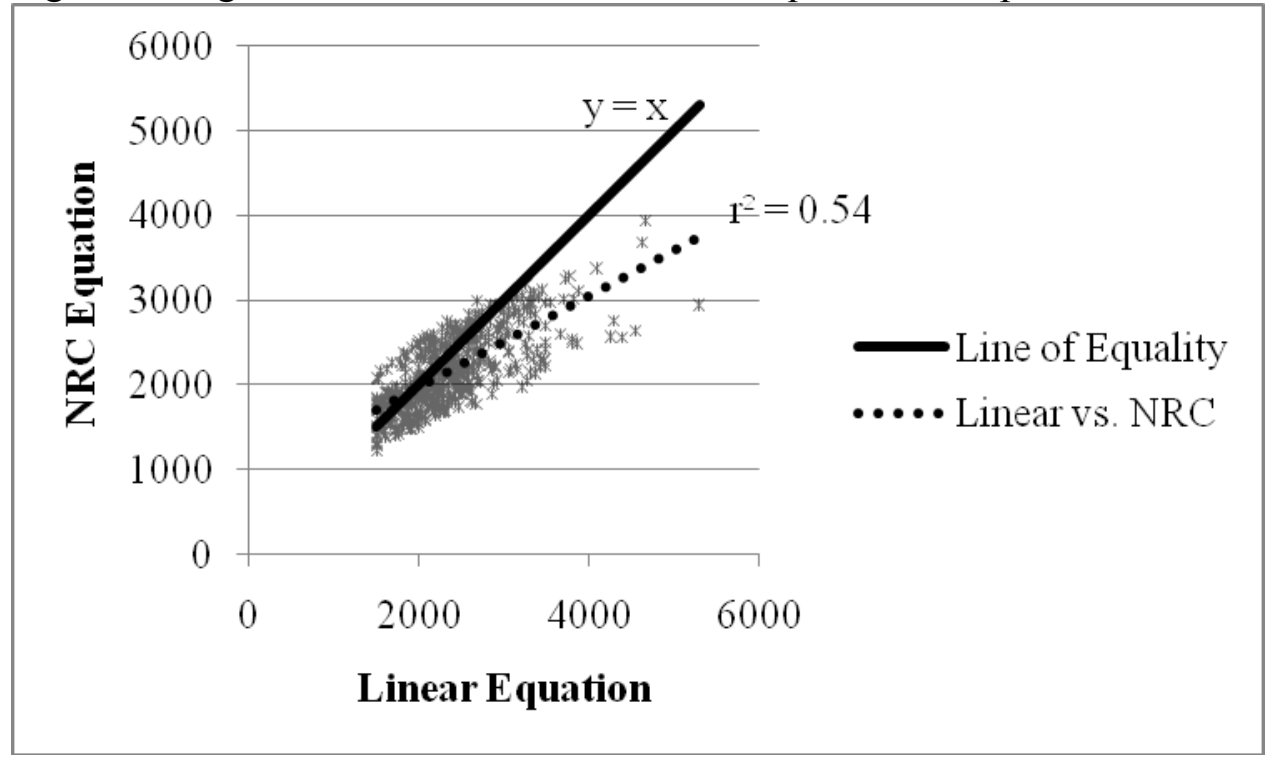

The regression of the Linear and NRC equations produced a strong correlation $(r=0.769$, $p<0.0001$ ), but criteria for agreement between the Linear and NRC equations were not met as $95 \%$ CI of the slope did not include one and 95\% CI of the intercept did not include zero. 
Figure 6. Regression of the Adjusted and Harris equations compared to the line of equality

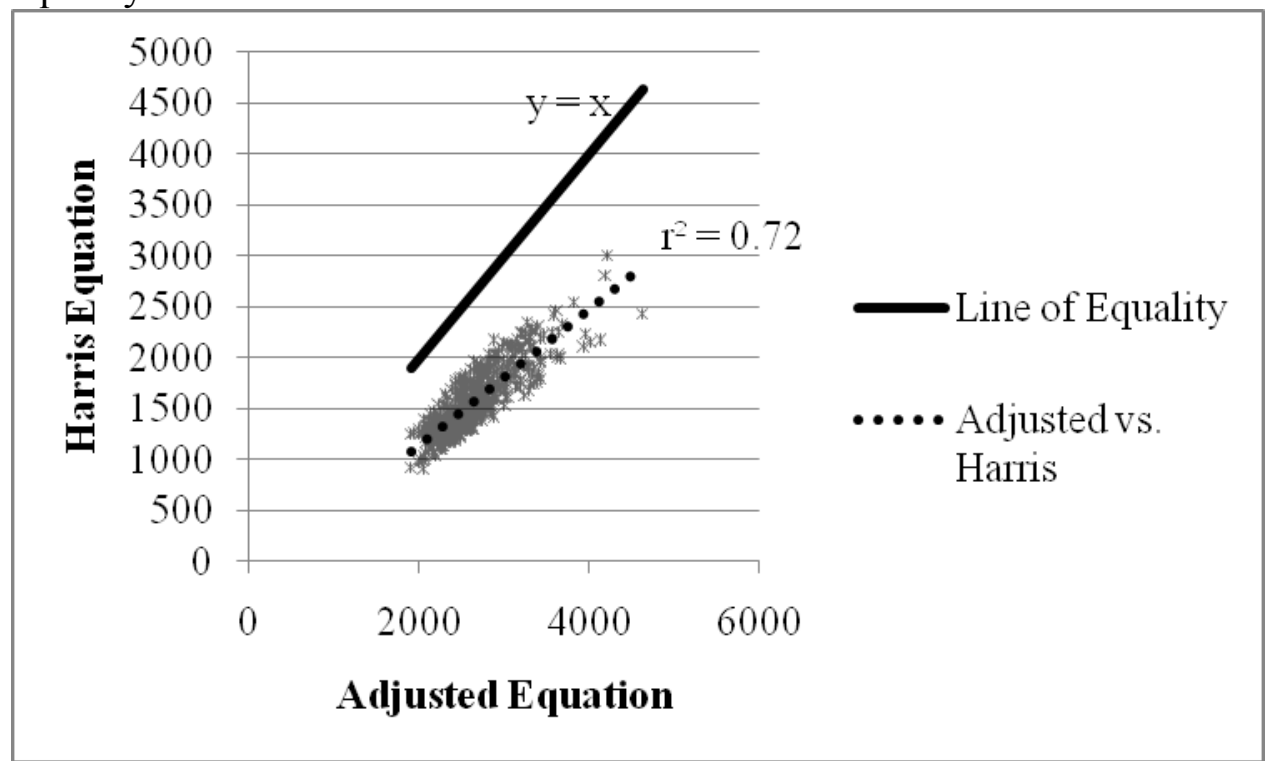

The regression of the Adjusted and Harris equations produced a strong correlation $(r=$ $0.869, p<0.0001)$, but the criteria for agreement between the Adjusted and Harris equations were not met as the $95 \% \mathrm{CI}$ of the slope did not include one and $95 \% \mathrm{CI}$ of the intercept did not include zero. 
Figure 7. Regression of the Adjusted and Mifflin equations compared to the line of equality

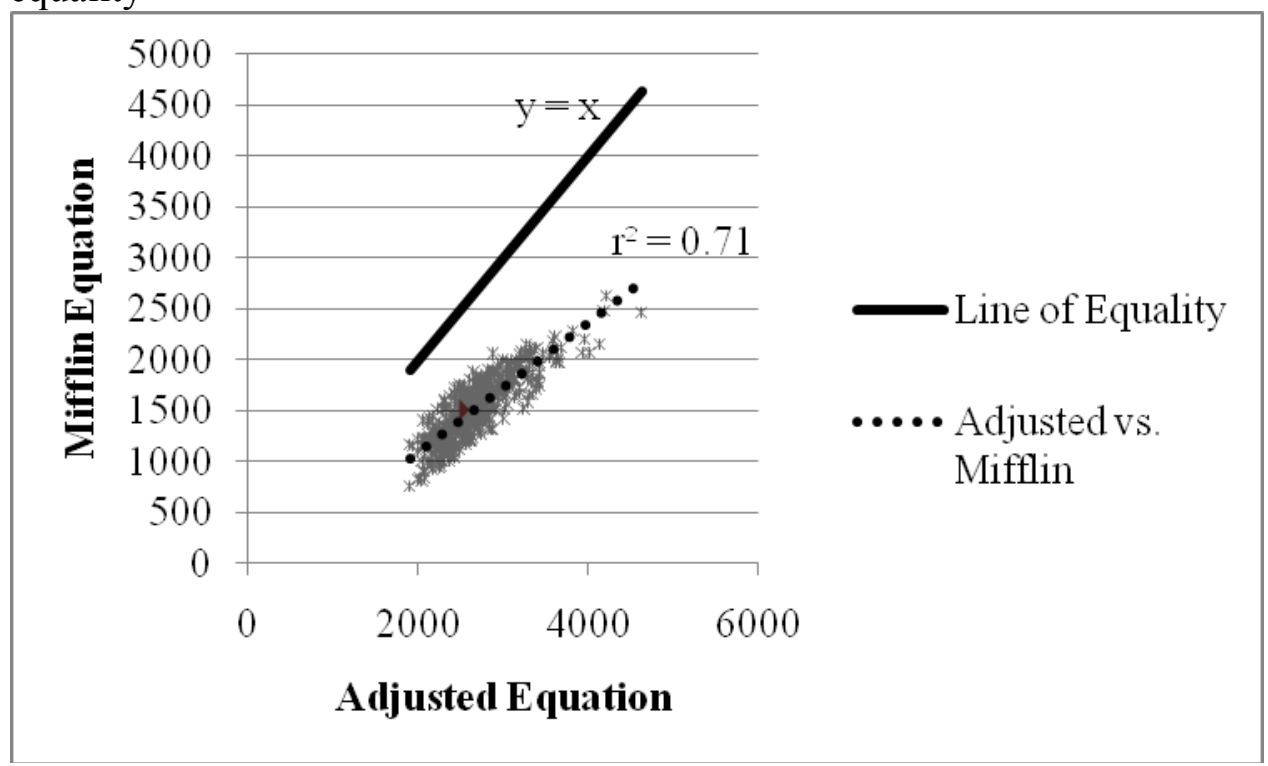

The regression of the Adjusted and Mifflin equations produced a strong correlation ( $r=$ $0.869, p<0.0001)$, but the criteria for agreement between the Adjusted and Mifflin equations were not met as the $95 \% \mathrm{CI}$ of the slope did not include one and $95 \% \mathrm{CI}$ of the intercept did not include zero. 
Figure 8. Regression of the Adjusted and NRC equations compared to the line of equality

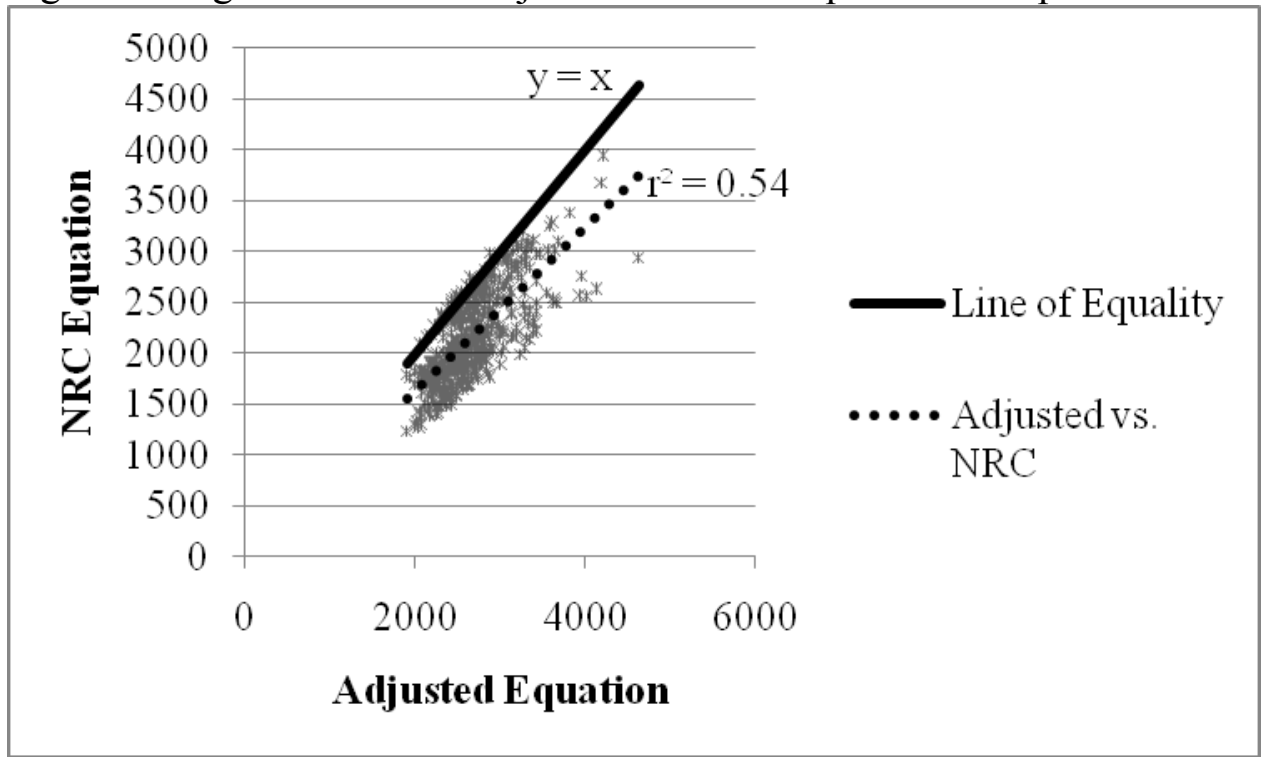

The regression of the Adjusted and NRC equations produced a strong correlation $(r=$ $0.770, p<0.0001)$, but the criteria for agreement between the Adjusted and NRC equations were not met as the $95 \%$ CI of the slope did not include one and $95 \%$ CI of the intercept did not include zero. 
Figure 9. Regression of the Harris and Mifflin equations compared to the line of equality

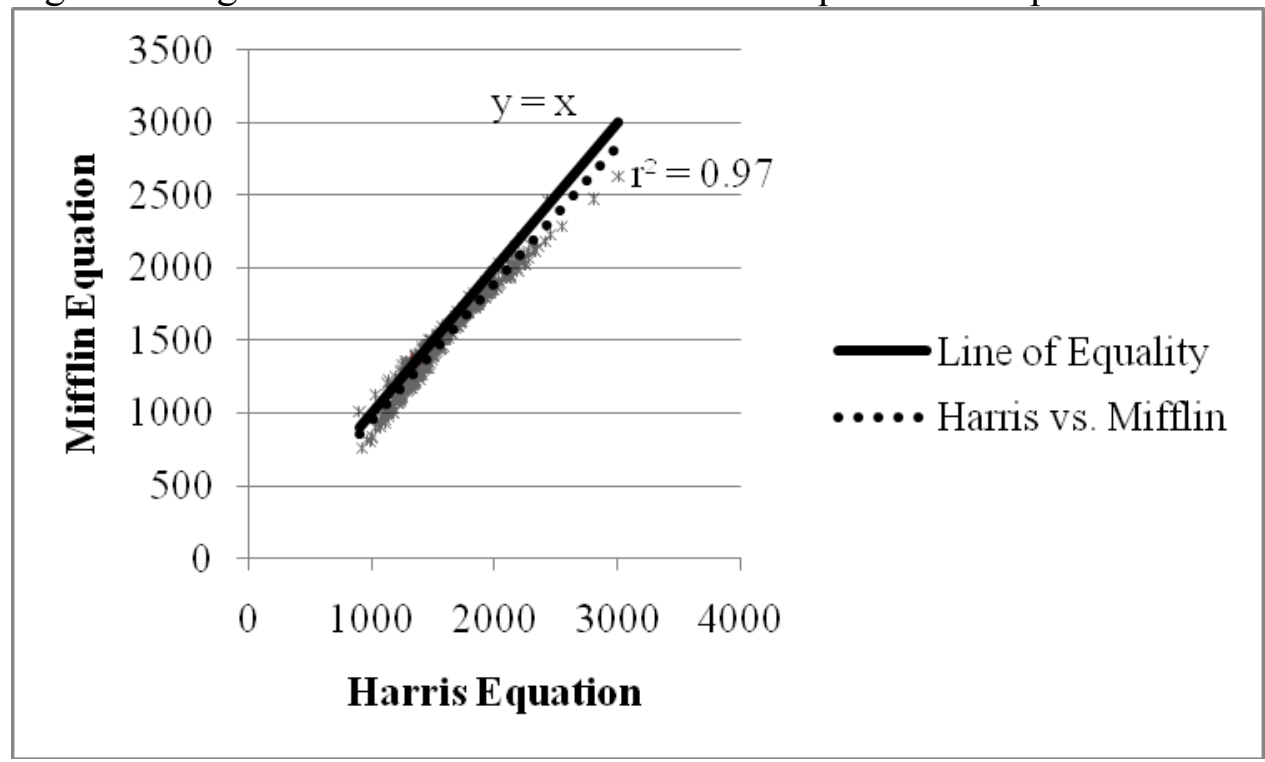

The regression of the Harris and Mifflin equations produced a strong correlation $(r=$ $0.986, p<0.0001)$. The Harris and Mifflin equation had a slope of $1.046(1.041,1.058)$ and an intercept of $7.71(-4.80,20.22)$. The criteria for agreement were not met. In this case, the slope did not include one and only the intercept included zero. 
Figure 10. Regression of the Harris and NRC equations compared to the line of equality

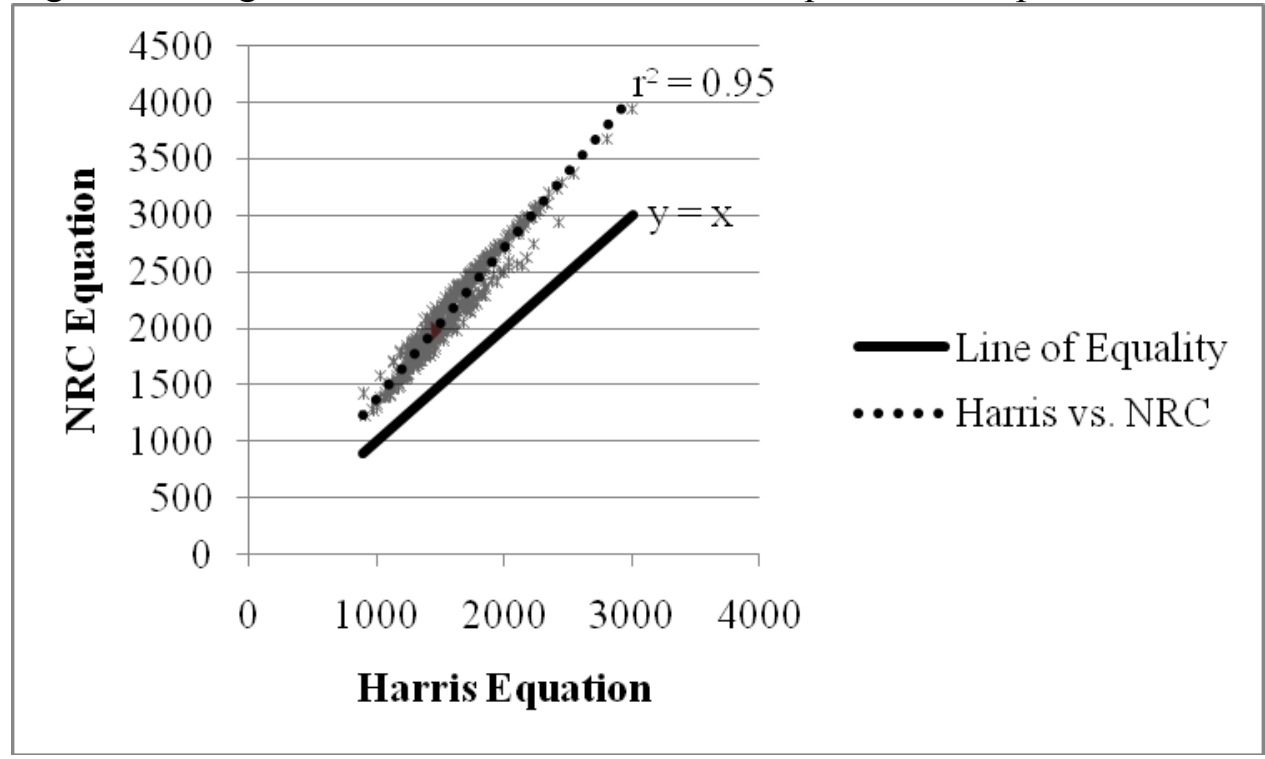

The regression of the Harris and NRC equations produced a strong correlation $(r=0.978$, $p<0.0001$ ), but the criteria for agreement between the Harris and NRC equations were not met as the $95 \%$ CI of the slope did not include one and $95 \%$ CI of the intercept did not include zero. 
Figure 11. Regression of the Mifflin and NRC equations compared to the line of equality

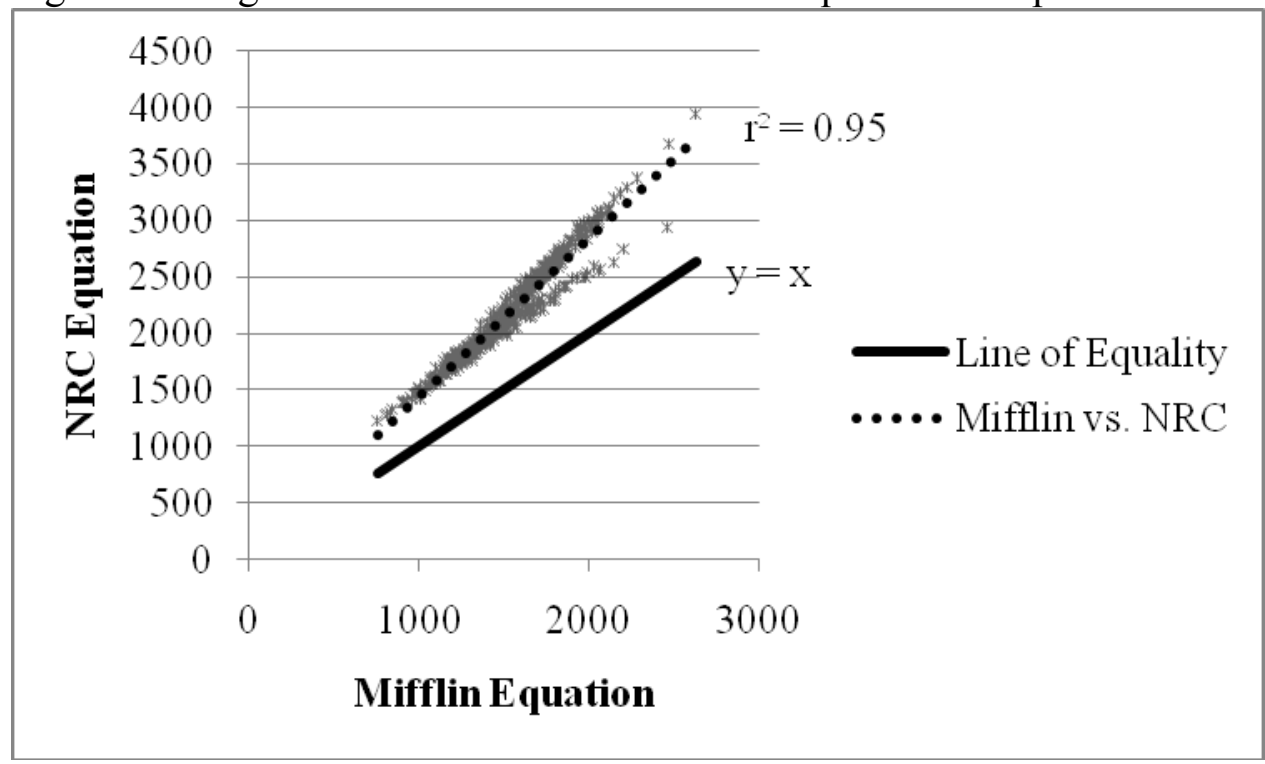

The regression of the Mifflin and NRC equations produced a strong correlation $(r=$ 0.977, $p<0.0001$ ), but the criteria for agreement between the Mifflin and NRC equations were not met as the regression line was too different from the $\mathrm{y}=\mathrm{x}$ line of equality

\section{$\underline{\text { Summary of specific aim } 1}$}

All simple regression analyses and bivariate correlations between the five EWR equations were statistically significant. However, none of the bivariate equations were statistically significant when compared to the line of equality. The estimates of water requirements among existing weight-based and energy-based equations do not agree with each other using the continuous NHANES 1999-2004 datasets. 


\section{Results of specific aim 2}

Specific aim 2: To determine agreement between existing equations used to estimate water requirements of adults and total water intake of adults using the continuous NHANES 1999-2004 datasets.

Hypothesis 2: Estimates of water requirements derived from commonly used weight-based and energy-based equations will lack agreement with total water intake data from the continuous NHANES 1999-2004 datasets.

The computations for each of the five EWR equations, as well as the TWI variable derived for this study are presented in Table 4. Five simple linear regressions were performed for each bivariate pairing of TWI on the EWR equations using the SAS proc surveyreg command. The findings of the simple regression analyses are presented in Table 7a and 7b. Square roots of the simple regression $\left(r^{2}\right)$ statistics were taken to derive a Pearson's linear correlation coefficient which defined the association between the bivariate pairs of variables (Table 6). Due to multiple comparisons the alpha was set at 0.01 . 
Table 7a. Simple linear regression to examine the $95 \%$ CI of the slope

\begin{tabular}{cccccccc}
\hline \multicolumn{2}{c}{ Variables } & & & \multicolumn{3}{c}{$95 \%$ CI of Slope } & \\
\cline { 1 - 1 } Y & X & $n$ & Slope & Lower & Upper & $r^{2}$ \\
\cline { 1 - 1 } TWI & Linear & 10,546 & 0.372 & 0.318 & 0.426 & 0.033 \\
TWI & Adjusted & 10,546 & 0.558 & 0.477 & 0.638 & 0.034 \\
TWI & Harris & 10,470 & 1.026 & 0.946 & 1.105 & 0.071 \\
TWI & Mifflin & 10,470 & 1.115 & 1.028 & 1.203 & 0.075 \\
TWI & NRC & 10,470 & 0.784 & 0.727 & 0.841 & 0.081
\end{tabular}

Note: $\mathrm{Y}=$ Dependent Variable (outcome); $\mathrm{X}=$ Independent Variable (predictor); Slope = Beta Unstandardized Coefficient; $\mathrm{CI}=$ Confidence Interval; Linear = Linear equation; Adjusted $=$ Adjusted equation; Mifflin $=$ Mifflin St. Jeor equation; Harris $=$ Harris Benedict equation; $\mathrm{NRC}=$ National Research Council equation; $\mathrm{TWI}=$ Total Water Intake. All model coefficients were statistically significant at $p<.0001$.

Table 7b. Simple linear regression to examine the $95 \%$ CI of the intercept

\begin{tabular}{cccccccc}
\hline \multicolumn{2}{c}{ Variables } & & & \multicolumn{3}{c}{$95 \%$ CI of Intercept } & \\
\cline { 1 - 1 } & Y & X & $n$ & Intercept & Lower & Upper & $r^{2}$ \\
\cline { 1 - 1 } TWI & Linear & 10,546 & $2,404.96$ & $2,283.15$ & $2,526.77$ & 0.033 \\
TWI & Adjusted & 10,546 & $1,793.29$ & $1,588.95$ & $1,997.63$ & 0.034 \\
& & & & & & \\
TWI & Harris & 10,470 & $1,633.79$ & $1,507.83$ & $1,759.74$ & 0.071 \\
TWI & Mifflin & 10,470 & $1,582.10$ & $1,452.32$ & $1,711.88$ & 0.075 \\
TWI & NRC & 10,470 & $1,570.83$ & $1,445.51$ & $1,696.14$ & 0.081
\end{tabular}

Note: $\mathrm{Y}=\overline{\text { Dependent Variable (outcome); } \mathrm{X}=\text { Independent Variable (predictor) }}$; $\mathrm{CI}=$ Confidence Interval; Linear $=$ Linear equation; Adjusted $=$ Adjusted equation; Mifflin $=$ Mifflin St. Jeor equation; Harris $=$ Harris Benedict equation; NRC $=$ National Research Council equation; TWI $=$ Total Water Intake. All model coefficients were statistically significant at $p<.0001$. 
All regression models and model coefficients were statistically significant at an alpha of $0.01(p<.0001)$. The model $r^{2}$ for all regressions involving the five EWR equations and TWI were small and ranged from 3.4\% (TWI regressed onto the Adjusted equation) to $8.1 \%$ (TWI regressed onto the NRC equation) (Table $7 \mathrm{a}$ and $7 \mathrm{~b}$ ). Less than $10 \%$ of the variability in TWI could be accounted for by the predictors. The regression of TWI on the NRC predictor returned the highest $r^{2}$ value $\left(r^{2}=0.081\right)$.

Results of the correlation analyses were presented in Table 6. Correlations between the bivariate EWR equations and TWI were all positive, but not strong. All correlation coefficients were less than 0.30 , the strongest being between TWI and NRC $(r$ $=0.285)$. However, the correlations were all statistically significant.

The results of the $95 \% \mathrm{CI}$ for the slope and the intercept can be viewed in table $7 \mathrm{a}$ and $7 \mathrm{~b}$. In these tables it is evident that the slope of the regression of TWI on the Harris equation was 1.026 and that the $95 \%$ CI of the slope does include one (CI 0.946, 1.105). The criteria for agreement were for both the $95 \%$ CI of the slope to include one and the 95\% CI of the intercept to include zero (Volaufova, 2005).

The intercept of the regression of TWI on the Harris equation was 1633.79, but the $95 \% \mathrm{CI}$ of this intercept did not include zero $(1507.83,1759.74)$. The regression of TWI on the Harris equation resulted in $r^{2}$ of 0.071 and $r=0.266$, which is a considerably weak correlation. Since the first criteria for agreement was not met here, it is unnecessary to consider the second criteria for agreement i.e., 95\% CI of the slope and intercept including one and zero, respectively. 


\section{$\underline{\text { Summary of specific aim } 2}$}

All simple regression analyses and bivariate correlations between the five EWR equations and TWI were statistically significant at the $p<0.0001$ level. However, the five EWR equations and TWI regression models and associations were weak. Estimated water requirements derived from commonly used weight-based and energy-based equations did not agree with TWI obtained from the continuous NHANES 1999-2004 datasets.

\section{$\underline{\text { Results of specific aim } 3}$}

Specific aim 3: To determine the correlation between fat-free mass (FFM) and total water intake using the continuous NHANES 1999-2004 data across the range of age and BMI.

Hypothesis 3: Bioelectrical impedance analysis (BIA) measures of fat-free mass and total water intake using the continuous NHANES 1999-2004 datasets will be positively correlated across the range of BMI.

The findings of the simple regression analyses are presented in Table 8. Square roots of the simple regression $r$-square statistics were taken to derive a Pearson's linear correlation coefficient which defined the association between BMI and TWI for each of the four BMI group regressions $(n=4,422)$. Results of the correlational analysis are also presented in Table 8 . The alpha was set at 0.05 for this aim. 
Table 8. Simple regression results for total water intake (TWI) outcome regressed on estimated fat-free mass according to BMI classification.

\begin{tabular}{|c|c|c|c|c|c|c|}
\hline \multirow[b]{2}{*}{ BMI Group $\left(\mathrm{kg} / \mathrm{m}^{2}\right)$} & \multirow[b]{2}{*}{$n$} & \multirow[b]{2}{*}{$\mathrm{B}$} & \multicolumn{2}{|c|}{$95 \% \mathrm{CI}$ for $\mathrm{B}$} & \multirow[b]{2}{*}{$r^{2}$} & \multirow[b]{2}{*}{$r$} \\
\hline & & & Lower & Upper & & \\
\hline$<18.5$ & 129 & 52.19 & 20.83 & 83.55 & .107 & .327 \\
\hline $18.5-24.9$ & 1,654 & 30.18 & 24.35 & 36.01 & .07 & .265 \\
\hline $25-29.9$ & 1,394 & 29.71 & 23.47 & 35.95 & .087 & .295 \\
\hline$\geq 30$ & 1,245 & 21.62 & 15.58 & 27.66 & .059 & .243 \\
\hline
\end{tabular}

Note: $\mathrm{BMI}=$ Body Mass Index; $\mathrm{B}=$ Unstandardized Coefficient; $\mathrm{CI}=$ Confidence Interval; $r^{2}=$ Coefficient of Multiple Determination; $r=$ Pearson's Linear Correlation Coefficient. All models and model coefficients were statistically significant at $p<.0001$.

All regression models and model coefficients were statistically significant $(p<$ .0001). All BMI groups had a positive relationship between BIDFFM and TWI, indicating that increases or decreases in the value of the BIDFFM are associated with movement in a like manner on the TWI variable.

The strongest correlation between BIDFFM and TWI was for the BMI group of $<$ $18.5(r=0.327, p<.0001)$. The coefficient of the regression was 52.19 , indicating that for a one unit increase in the BIDFFM variable, TWI increases by $52.19 \mathrm{ml}$.

$\underline{\text { Summary of specific aim } 3}$

There is sufficient albeit not strong evidence to indicate a statistically significant relationship between the TWI and BIDFFM variables across all four categories of BMI. The BIA measures of estimated FFM and TWI from the continuous NHANES 1999-2004 datasets are positively correlated across the range of BMI and there is justification for using FFM as a predictor of TWI. 


\section{Results of specific aim 4}

Specific aim 4: To derive and validate one or more practical equations for predicting total water intake using the continuous NHANES 1999-2004 datasets.

\section{Variable selection}

The univariate analysis for all clinically significant numeric variables is depicted in Table 9. The alpha level was set at 0.05 for this aim. Most of the selected variables were significant at $p<0.0001$ and were therefore eligible to be entered into the full model A and full model B. Body Mass Index $(p<0.002)$ was also eligible for entry into the models. 
Table 9. Univariate analysis of numeric variables with total water intake

\begin{tabular}{cccccccc}
\hline & & & & \multicolumn{2}{c}{$95 \%$ CI } & & \\
\cline { 5 - 6 } Variable & $n$ & Slope & $S E$ & Lower & Upper & $t$ & $p$ \\
\hline Age & 10,732 & -7.81 & 0.69 & -9.20 & -6.42 & -11.36 & $<0.0001$ \\
Kcals & 10,732 & 0.62 & 0.01 & 0.59 & 0.65 & 42.09 & $<0.0001$ \\
Weight & 10,546 & 11.15 & 0.80 & 9.55 & 12.76 & 14.00 & $<0.0001$ \\
Height & 10,535 & 35.00 & 1.31 & 32.35 & 37.62 & 26.65 & $<0.0001$ \\
FFM & 4,480 & 23.16 & 1.36 & 20.42 & 25.90 & 17.05 & $<0.0001$ \\
BMI & 10,470 & 9.70 & 3.01 & 3.63 & 15.77 & 3.22 & 0.0024 \\
Osmolality & 10,147 & -14.08 & 2.88 & -19.89 & -8.27 & -4.88 & $<0.0001$
\end{tabular}

Note: FFM = Fat-Free Mass; $\mathrm{CI}=$ Confidence Interval; TWI =Total Water Intake

There was a suspicion of multicollinearity and confounding from the overlapping types of variables included in the models, but these types of problems were not unexpected and were tested. These variables, along with the clinically relevant categorical variables of race/ethnicity and gender, were therefore eligible for entry into the full model A and B regressions.

\section{Building multiple regression model A with FFM}

Total water intake was the outcome variable in the computation of each multiple regression analyses for all data. Fat-free mass was only collected on those aged 19-49 and therefore the sample size for model A with FFM was 4,480 while sample size was 10,468 when FFM was not in the model (model B). The findings of the full regression model A before backward elimination was initiated are presented in Table 10. The table includes the $F$-statistic and $p$-values as well as the VIF values for the nine predictor variables. Full 
model A was significant $F(12,44)=87.05, p<.0001$ with an $r^{2}$ of 0.266 . Twenty seven percent of the variability in TWI could be explained by the nine predictor variables. The root mean square error (RMSE) of full model A was 1,637.85.

Table 10. Test of model A effects on full regression ${ }^{\mathrm{a}}$

\begin{tabular}{lccc}
\hline Variable & $\boldsymbol{F}$ value & $\boldsymbol{p}$-value & VIF \\
\hline Race/ethnicity & 29.01 & $<0.0001$ & 1.12 \\
Kcals & 413.50 & $<0.0001$ & 1.22 \\
Age & 5.60 & 0.023 & 1.03 \\
Height* & 1.02 & 0.317 & 27.86 \\
Weight* & 4.03 & 0.051 & 113.64 \\
FFM & 6.45 & $<0.015$ & 7.28 \\
Gender & 1.76 & 0.192 & 3.43 \\
BMI* & 3.95 & 0.053 & 84.03
\end{tabular}

\begin{tabular}{llll} 
Osmolality & 0.40 & 0.531 & 1.09 \\
\hline
\end{tabular}

Note: VIF $=$ Variance Inflation Factor. * Variable with a VIF $>10$. Highest VIF removed from subsequent model. FFM $=$ Fat-Free Mass. Bolded variables were statistically significant at $p<0.05$. ${ }^{a}$ model significant at $p<0.0001$. 
The findings of the unstandardized model coefficients (B), standard error, 95\% CI for the coefficients, the $t$-statistics and significance values for the nine predictor variables were presented in Table 11. The VIF of weight, BMI and height were $>10$ (Table 10). The VIF of the weight variable was the highest at 113.64 and was therefore removed from the full model A. The variables of race/ethnicity, kcals, age, height, FFM, gender, BMI, and osmolality were retained because the backwards elimination approach sequentially removed one variable at a time from the full model A. In this step the weight variable was removed based on having the highest VIF. 
Table 11. Full model A of estimated coefficients and significance

\begin{tabular}{|c|c|c|c|c|c|c|}
\hline \multirow[b]{2}{*}{ Variable } & \multirow[b]{2}{*}{ Coefficient } & \multirow[b]{2}{*}{$S E$} & \multicolumn{2}{|c|}{$95 \%$ CI } & \multirow[b]{2}{*}{$t$} & \multirow[b]{2}{*}{$p$-value } \\
\hline & & & Lower & Upper & & \\
\hline \multicolumn{7}{|l|}{ Race/ethnicity } \\
\hline Mexican American & -424.21 & 167.54 & -761.86 & -86.56 & -2.53 & 0.015 \\
\hline Other Hispanic & -606.51 & 198.02 & $-1,005.58$ & -207.43 & -3.06 & 0.004 \\
\hline Non-Hispanic White & -307.32 & 167.14 & -644.16 & 29.52 & -1.84 & 0.073 \\
\hline Non-Hispanic Black & -997.72 & 177.93 & $-1,356.31$ & -639.13 & -5.61 & $<0.0001$ \\
\hline Multi-Racial/Other & - & 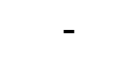 & 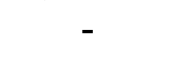 & - & - & - \\
\hline Kcals & 0.78 & 0.04 & 0.70 & 0.86 & 20.33 & $<0.0001$ \\
\hline Age & 9.86 & 4.17 & 1.46 & 18.25 & 2.37 & 0.023 \\
\hline Height & -15.70 & 15.52 & -46.98 & 15.57 & -1.01 & 0.32 \\
\hline Weight & 31.71 & 15.79 & -0.13 & 63.50 & 2.01 & 0.050 \\
\hline FFM & 14.47 & 5.69 & 2.99 & 25.94 & 2.54 & 0.010 \\
\hline \multicolumn{7}{|l|}{ Gender } \\
\hline Male & -137.84 & 103.95 & -347.33 & 71.65 & -1.33 & 0.190 \\
\hline Female & - & - & - & - & - & - \\
\hline BMI & -86.22 & 43.40 & -173.66 & 1.23 & -1.99 & 0.050 \\
\hline Osmolality & -5.61 & 8.88 & -23.50 & 12.28 & -0.63 & 0.530 \\
\hline
\end{tabular}

Note: Bolded variables are significant at $\mathrm{p}<0.05$. FFM $=$ Fat-Free Mass.

The results of the second regression on the remaining variables and their significance are depicted in Table 12. The results of model A after the second regression were significant $F(11,44)=93.17, p<.0001$ with an $r^{2}$ of 0.265 . Twenty seven percent of the variability in TWI could be explained by the eight predictor variables. The RMSE of the second regression model A was 1,638.55. 
Table 12. Model A effects on regression without weight ${ }^{\mathrm{a}}$

\begin{tabular}{lccc}
\hline Variable & $\boldsymbol{F}$ value & $\boldsymbol{p}$-value & VIF \\
\hline Race/ethnicity & 27.55 & $<0.0001$ & 1.12 \\
Kcals & 413.90 & $<0.0001$ & 1.22 \\
Age & 6.22 & 0.017 & 1.03 \\
Height & 4.21 & 0.046 & 3.11 \\
FFM & 8.78 & 0.005 & 6.67 \\
Gender & 2.58 & 0.115 & 3.36 \\
BMI & 0.01 & 0.916 & 2.84 \\
Osmolality & 0.42 & 0.523 & 1.08 \\
\hline
\end{tabular}

Note: $\mathrm{FFM}=$ Fat-Free Mass, VIF $=$ Variance Inflation Factor.

${ }^{\mathrm{a} B}$ Bolded variables were statistically significant at $p<0.05$.

The VIF of the remaining eight variables was $<10$ (Table 12). The body weight variable was removed due to the presence of multicollinearity resulting in a large change $(>25 \%)$ in coefficients and $p$-values. The findings of the unstandardized model coefficients (B), standard error, $95 \%$ CI for the coefficients, the $t$-statistics and significance values for the eight predictor variables are depicted in Table 13.

The BMI variable had the highest $p$-value of 0.916 and was therefore the next variable to be removed from model A. The seven variables of race/ethnicity, kcals, age, height, FFM, gender, and osmolality were retained because a sequential removal from model A was accomplished one variable at a time with the backwards elimination approach. In this step the BMI variable was removed based on having the highest $p$ value. 
Table 13. Model A of estimated coefficients and significance without weight

\begin{tabular}{|c|c|c|c|c|c|c|}
\hline \multirow[b]{2}{*}{ Variable } & \multirow[b]{2}{*}{ Coefficient } & \multirow[b]{2}{*}{$S E$} & \multicolumn{2}{|c|}{ 95\% CI } & \multirow[b]{2}{*}{$t$} & \multirow[b]{2}{*}{$p$-value } \\
\hline & & & Lower & Upper & & \\
\hline \multicolumn{7}{|l|}{ Race/ethnicity } \\
\hline Mexican American & -448.72 & 172.02 & -795.41 & -102.04 & -2.61 & 0.012 \\
\hline Other Hispanic & -635.08 & 199.78 & $-1,037.71$ & -232.46 & -3.18 & 0.003 \\
\hline Non-Hispanic White & -330.75 & 169.88 & -673.13 & 11.62 & -1.95 & 0.058 \\
\hline Non-Hispanic Black & $-1,027.89$ & 183.68 & $-1,398.07$ & -657.71 & -5.80 & $<0.0001$ \\
\hline Multi-Racial/Other & & 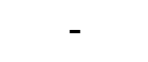 & & . & - & 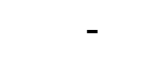 \\
\hline Kcals & 0.78 & 0.04 & 0.70 & 0.86 & 20.34 & $<0.0001$ \\
\hline Age & 10.23 & 4.10 & 1.96 & 18.50 & 2.49 & 0.017 \\
\hline Height & 11.87 & 5.78 & 0.21 & 23.52 & 2.05 & 0.046 \\
\hline FFM & 17.41 & 5.87 & 5.57 & 29.24 & 2.96 & 0.0049 \\
\hline \multicolumn{7}{|l|}{ Gender } \\
\hline Male & -169.18 & 105.29 & -381.37 & 43.02 & -1.61 & 0.115 \\
\hline Female & - & 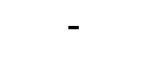 & - & - & - & - \\
\hline BMI & 0.93 & 8.69 & -18.44 & 16.59 & -0.11 & 0.916 \\
\hline Osmolality & -5.72 & 8.87 & -23.59 & 12.16 & -0.64 & 0.523 \\
\hline
\end{tabular}

Note: Bolded variables are significant at $p<0.05$. FFM $=$ Fat-Free Mass.

The results of the third regression on the remaining variables and their significance are depicted in Table 14. The results of model A after the third regression were significant $F(10,44)=100.97, p<.0001$ with an $r^{2}$ of 0.265 . Twenty seven percent of the variability in TWI could be explained by the seven predictor variables. The RMSE of the third model A was $1,637.80$. The VIF of the remaining seven variables was $<10$ (Table 14). 
Table 14. Model A effects on regression without BMI ${ }^{\mathrm{a}}$

\begin{tabular}{lccc}
\hline Variable & $\boldsymbol{F}$ value & $\boldsymbol{p}$-value & VIF \\
\hline Race/ethnicity & 27.83 & $<0.0001$ & 1.12 \\
Kcals & 411.49 & $<0.0001$ & 1.22 \\
Age & 5.98 & 0.019 & 1.02 \\
Height & 7.48 & 0.009 & 2.39 \\
FFM & 19.02 & $<0.001$ & 2.42 \\
Gender & 3.16 & 0.082 & 2.45 \\
Osmolality & 0.40 & 0.530 & 1.08 \\
\hline
\end{tabular}

Note: FFM = Fat-Free Mass. VIF= Variance Inflation Factor.

${ }^{\mathrm{a}}$ Bolded variables were statistically significant at $p<0.05$.

The findings of the unstandardized model coefficients (B), standard error, 95\% CI for the coefficients, the $t$-statistics and significance values for the seven predictor variables are presented in Table 15. There was no evidence of confounding since none of the coefficients were changed by greater than $20-25 \%$ before and after removal of the BMI variable and the $p$-value did not change in terms of significance.

The osmolality variable had the highest $p$-value of 0.530 and was therefore the next variable to be removed from model A. The six variables of race/ethnicity, kcals, age, height, FFM, and gender were retained because the backwards elimination approach entailed a sequential removal from model $\mathrm{A}$ of one variable at a time. In this step the osmolality variable was removed based on having the highest $p$-value. 
Table 15. Model A of estimated coefficients and significance without BMI

\begin{tabular}{|c|c|c|c|c|c|c|}
\hline \multirow[b]{2}{*}{ Variable } & \multirow[b]{2}{*}{ Coefficient } & \multirow[b]{2}{*}{$S E$} & \multicolumn{2}{|c|}{$95 \% \mathrm{CI}$} & \multirow[b]{2}{*}{$t$} & \multirow[b]{2}{*}{$p$-value } \\
\hline & & & Lower & Upper & & \\
\hline \multicolumn{7}{|l|}{ Race/ethnicity } \\
\hline Mexican American & -452.97 & 172.46 & -800.55 & -105.39 & -2.63 & 0.012 \\
\hline Other Hispanic & -638.84 & 199.13 & $-1,040.16$ & -237.53 & -3.21 & 0.003 \\
\hline Non-Hispanic White & -331.62 & 169.69 & -673.61 & 10.37 & -1.95 & 0.057 \\
\hline Non-Hispanic Black & $-1,027.33$ & 183.61 & $-1,395.36$ & -659.31 & -5.63 & $<0.0001$ \\
\hline Multi-Racial/Other & - & - & - & - & - & - \\
\hline Kcals & 0.78 & 0.04 & 0.70 & 0.86 & 20.29 & $<0.0001$ \\
\hline Age & 10.24 & 4.19 & 1.80 & 18.68 & 2.45 & 0.019 \\
\hline Height & 12.17 & 4.45 & 3.20 & 21.15 & 2.73 & 0.009 \\
\hline FFM & 16.98 & 3.89 & 9.13 & 24.83 & 4.36 & $<0.001$ \\
\hline \multicolumn{7}{|l|}{ Gender } \\
\hline Male & -165.10 & 92.84 & -352.21 & 22.02 & -1.78 & 0.082 \\
\hline Female & - & - & - & - & - & - \\
\hline Osmolality & -5.60 & 8.84 & -23.42 & 12.22 & -0.63 & 0.530 \\
\hline
\end{tabular}

Note: Bolded variables were statistically significant at $p<0.05$. FFM $=$ fat-free mass

The results of the fourth regression on the remaining variables and their significance are portrayed in Table 16. The results of model A after the fourth regression were significant $F(9,44)=111.27, p<.0001$ with an $r^{2}$ of .0269 . Twenty seven percent of the variability in TWI could be explained by the six predictor variables. The RMSE of the model was 1,627.56. The VIF of the remaining six variables was $<10$ (Table 16). 
Table 16. Final model A effects of regression ${ }^{\mathrm{a}}$

\begin{tabular}{lccc}
\hline Variable & $\boldsymbol{F}$ value & $\boldsymbol{p}$-value & VIF \\
\hline Race/ethnicity & 31.64 & $<0.0001$ & 1.12 \\
Kcals & 422.98 & $<0.0001$ & 1.22 \\
Age & 6.51 & 0.014 & 1.01 \\
Height & 6.67 & 0.013 & 2.39 \\
FFM & 23.99 & $<0.001$ & 2.39 \\
Gender & 3.98 & 0.048 & 2.37 \\
\hline
\end{tabular}

Note: FFM = Fat-Free Mass. VIF= Variance Inflation Factor.

${ }^{\mathrm{a}}$ All variables were statistically significant at $p<0.05$.

The findings of the unstandardized model coefficients (B), standard error, 95\% CI for the coefficients, the $t$-statistics and significance values for the six predictor variables are illustrated in Table 17. There was no evidence of confounding since none of the coefficients were changed by greater than $20-25 \%$ before and after removal of the osmolality variable and the $p$-value did not change in terms of significance.

Since none of the remaining variables had a $p$-value $>0.05$, no more variables were removed. The variables of race/ethnicity, kcals, age, height, FFM, and gender were retained as final model A because this model had the highest $F$ value, the lowest $R M S E$, and all of the remaining variables were significant. The final equation for model A was:

Total Water Intake $=-721.87-450.73$ Mexican American - 622.27 Other Hispanic - 320.45 Non-Hispanic White - 1,021.67 Non-Hispanic Black +0.78 Kcals +10.24 Age +11.00 Height +17.93 FFM - 176.37 Male. 
Table 17. Final model A of estimated coefficients and significance without osmolality

\begin{tabular}{|c|c|c|c|c|c|c|}
\hline \multirow[b]{2}{*}{ Variable } & \multirow[b]{2}{*}{ Coefficient } & \multirow[b]{2}{*}{$S E$} & \multicolumn{2}{|c|}{$95 \% \mathrm{CI}$} & \multirow[b]{2}{*}{$t$} & \multirow[b]{2}{*}{$p$-value } \\
\hline & & & Lower & Upper & & \\
\hline \multicolumn{7}{|l|}{ Race/ethnicity } \\
\hline Mexican American & -450.73 & 168.90 & -791.13 & -110.34 & -2.67 & 0.011 \\
\hline Other Hispanic & -622.27 & 194.24 & $-1,013.75$ & -110.34 & -3.20 & 0.003 \\
\hline Non-Hispanic White & -320.45 & 161.70 & -646.34 & 5.45 & -1.98 & 0.054 \\
\hline Non-Hispanic Black & $-1,021.67$ & 168.92 & $-1,362.10$ & -681.24 & -6.05 & $<0.0001$ \\
\hline Multi-Racial/Other & - & - & - & - & - & - \\
\hline Kcals & 0.78 & 0.04 & 0.70 & 0.85 & 20.57 & $<0.0001$ \\
\hline Age & 10.24 & 4.01 & 2.15 & 18.33 & 2.55 & 0.014 \\
\hline Height & 11.00 & 4.26 & 2.42 & 19.59 & 2.58 & 0.013 \\
\hline FFM & 17.93 & 3.66 & 10.55 & 25.30 & 4.90 & $<0.001$ \\
\hline \multicolumn{7}{|l|}{ Gender } \\
\hline Male & -176.37 & 88.99 & -355.71 & -7.51 & -3.71 & 0.048 \\
\hline Female & - & - & - & - & - & - \\
\hline
\end{tabular}

\section{$\underline{\text { Building multiple regression model B without FFM }}$}

The findings of the unstandardized model coefficients $(B)$, standard error, 95\% CI for the coefficients, the $t$-statistics and significance values for the eight predictor variables are presented in Table 18. The table includes the F-statistic and p-values as well as the VIF values. Full model B was significant $F(11,44)=140.91, p<.0001$ with an $r^{2}$ of 0.282 . Twenty eight percent of the variability in TWI could be explained by the eight predictor variables. The RMSE of the model was 1,473.0. 
Table 18. Test of model B effects on full regression ${ }^{\mathrm{a}}$

\begin{tabular}{llll}
\hline Variable & $\boldsymbol{F}$ value & $\boldsymbol{p}$-value & VIF \\
\hline Race/ethnicity & 34.37 & $<0.0001$ & 1.08 \\
Kcals & 902.37 & $<0.0001$ & 5.18 \\
Age & 5.87 & 0.02 & 1.16 \\
Height & 0 & 0.98 & $22.73^{*}$ \\
Weight & 4.10 & 0.05 & $90.91^{*}$ \\
Gender & 0 & 0.95 & 1.72 \\
BMI & 2.07 & 0.116 & $70.42^{*}$
\end{tabular}

Note: VIF $=$ Variance Inflation Factor.* Variable with a VIF $>10$. Highest VIF removed from subsequent model. ${ }^{\mathrm{a} B o l d e d}$ variables were statistically significant at $p<0.05$.

The findings of the unstandardized model coefficients (B), standard error, 95\% CI for the coefficients, the $t$-statistics and significance values for the eight predictor variables are presented in Table 19. Similar to the full model with FFM, the VIF of weight, BMI and height were $>10$ (Table 18). The VIF of the weight variable was the highest at 90.91 and was therefore removed from the full model B. The seven variables of race/ethnicity, kcals, age, height, gender, BMI, and osmolality were retained because the backwards elimination approach employed a sequential removal from full model B one variable at a time. In this step the weight variable was removed based on having the highest VIF. 
Table 19. Full model B of estimated coefficients and significance

\begin{tabular}{|c|c|c|c|c|c|c|}
\hline \multirow[b]{2}{*}{ Variable } & \multirow[b]{2}{*}{ Coefficient } & \multirow[b]{2}{*}{$S E$} & \multicolumn{2}{|c|}{$95 \%$ CI } & \multirow[b]{2}{*}{$t$} & \multirow[b]{2}{*}{$p$-value } \\
\hline & & & Lower & Upper & & \\
\hline \multicolumn{7}{|l|}{ Race/ethnicity } \\
\hline Mexican American & -324.16 & 104.37 & -534.52 & -113.81 & -3.11 & 0.003 \\
\hline Other Hispanic & -391.87 & 129.81 & -653.48 & -130.25 & -3.02 & 0.004 \\
\hline Non-Hispanic White & -176.75 & 100.49 & -379.27 & 25.77 & -1.76 & 0.090 \\
\hline Non-Hispanic Black & -731.96 & 102.22 & -937.99 & -525.93 & -7.16 & $<0.0001$ \\
\hline Multi-Racial/Other & - & - & - & - & - & - \\
\hline Kcals & 0.75 & 0.03 & 0.70 & 0.81 & 30.04 & $<0.0001$ \\
\hline Age & -3.00 & 1.24 & -5.50 & -0.50 & -2.42 & 0.02 \\
\hline Height & -0.27 & 11.15 & -22.74 & 22.21 & -0.02 & 0.98 \\
\hline Weight & 23.76 & 11.73 & 0.12 & 47.39 & 2.03 & 0.05 \\
\hline \multicolumn{7}{|l|}{ Gender } \\
\hline Male & -3.08 & 52.09 & -108.07 & 101.90 & -0.06 & 0.95 \\
\hline Female & - & - & - & - & - & - \\
\hline BMI & -47.48 & 33.03 & -114.04 & 19.08 & 6.60 & 0.16 \\
\hline Osmolality & -17.36 & 5.14 & -27.72 & -7.00 & -3.43 & 0.002 \\
\hline
\end{tabular}

Note: Bolded variables were statistically significant at $p<0.05$.

The findings of the second regression after the elimination of the weight variable are presented in Table 20. The VIF was $<10$ for all remaining variables. The weight variable was removed due to the presence of multicollinearity causing a large change $(>25 \%)$ in coefficients and $p$-values.

The second regression model B was significant $F(10,44)=154.75, p<.0001$ with an $r^{2}$ of 0.281 . Twenty eight percent of the variability in TWI could be explained by the seven predictor variables. The RMSE of the model was 1,473.88. 
Table 20. Model B effects on regression without weight

\begin{tabular}{llll}
\hline Variable & $\boldsymbol{F}$ value & $\boldsymbol{p}$-value & VIF \\
\hline Race/ethnicity & 33.53 & $<0.0001$ & 1.08 \\
Kcals & 911.37 & $<0.0001$ & 1.26 \\
Age & 5.73 & 0.020 & 1.16 \\
Height & 82.44 & $<0.0001$ & 2.08 \\
Gender & 0 & 0.98 & 1.70 \\
BMI & 43.56 & $<0.0001$ & 1.01 \\
Osmolality & 11.75 & 0.001 & 1.10 \\
\hline
\end{tabular}

Note: VIF $=$ Variance Inflation Factor. ${ }^{a}$ Bolded variables were statistically significant at $p$ $<0.05$.

The findings of the unstandardized model coefficients (B), standard error, 95\% CI for the coefficients, the $t$-statistics and significance values for the seven predictor variables are displayed in Table 21 . The gender variable had the highest $p$-value of 0.98 and was therefore the next variable to be removed from model $\mathrm{B}$. The variables of race/ethnicity, kcals, age, height, BMI, and osmolality were retained because a sequential removal from the model is accomplished one variable at a time with the backwards elimination approach. In this step the gender variable was removed based on having the highest $p$-value. 
Table 21. Model B of estimated coefficients and significance without weight

\begin{tabular}{|c|c|c|c|c|c|c|}
\hline \multirow[b]{2}{*}{ Variable } & \multirow[b]{2}{*}{ Coefficient } & \multirow[b]{2}{*}{$S E$} & \multicolumn{2}{|c|}{$95 \% \mathrm{CI}$} & \multirow[b]{2}{*}{$t$} & \multirow[b]{2}{*}{$p$-value } \\
\hline & & & Lower & Upper & & \\
\hline \multicolumn{7}{|l|}{ Race/ethnicity } \\
\hline Mexican American & -342.76 & 105.78 & -555.94 & -129.58 & -3.24 & 0.002 \\
\hline Other Hispanic & -409.40 & 130.40 & -672.21 & -146.59 & -3.14 & 0.003 \\
\hline Non-Hispanic White & -193.47 & 102.66 & -400.37 & 13.43 & -1.88 & 0.066 \\
\hline Non-Hispanic Black & -755.04 & 106.16 & -968.99 & -541.10 & -7.11 & $<0.0001$ \\
\hline Multi-Racial/Other & - & - & - & - & - & - \\
\hline Kcals & 0.75 & 0.02 & 0.70 & 0.80 & 30.19 & $<0.0001$ \\
\hline Age & -2.94 & 1.23 & -5.41 & -0.46 & -2.39 & 0.02 \\
\hline Height & 22.34 & 2.46 & 17.38 & 27.30 & 9.08 & $<0.0001$ \\
\hline \multicolumn{7}{|l|}{ Gender } \\
\hline Male & -1.57 & 52.00 & -106.38 & 103.25 & -0.03 & 0.98 \\
\hline Female & - & - & - & - & - & - \\
\hline BMI & 19.83 & 3.00 & 13.78 & 25.89 & 6.60 & $<0.0001$ \\
\hline Osmolality & -17.50 & 5.10 & -27.79 & -7.21 & -3.43 & 0.001 \\
\hline
\end{tabular}

Note: Bolded variables were statistically significant at $p<0.05$.

The findings of the regression model $\mathrm{B}$ after the elimination of the gender variable are presented in Table 22 . The VIF was $<10$ for all remaining variables.

Model B was still significant $F(9,44)=166.12, p<.0001$ with an $r^{2}$ of 0.281 . Twenty eight percent of the variability in TWI could be explained by the six predictor variables. The RMSE of the model was $1,473.84$. 
Table 22. Final model B effects of regression ${ }^{\mathrm{a}}$

\begin{tabular}{llll}
\hline Variable & $\boldsymbol{F}$ value & $\boldsymbol{p}$-value & VIF \\
\hline Race/ethnicity & 33.39 & $<0.0001$ & 1.05 \\
Kcals & 977.27 & $<0.0001$ & 1.22 \\
Age & 5.67 & 0.020 & 1.14 \\
Height & 217.17 & $<0.0001$ & 1.21 \\
BMI & 43.37 & $<0.0001$ & 1.00
\end{tabular}

\begin{tabular}{llll} 
Osmolality & 12.13 & 0.001 & 1.09 \\
\hline
\end{tabular}

$\mathrm{VIF}=$ Variance Inflation Factor. ${ }^{\mathrm{a}} \mathrm{All}$ variables were statistically significant at $p<0.05$.

The findings of the unstandardized model coefficients $(B)$, standard error, $95 \% \mathrm{CI}$ for the coefficients, the $t$-statistics and significance values for the six predictor variables are presented in Table 23. There was no evidence of confounding since none of the coefficients were changed by greater than $20-25 \%$ before and after removal of the gender variable and the $p$-value did not change in terms of significance.

Since none of the remaining variables had a $p$-value $>0.05$, no more variables were removed from model B. Therefore, the variables of race/ethnicity, kcals, age, height, BMI, and osmolality were retained as the final model because this model had the highest $F$ value, the lowest $R M S E$, and all of the remaining variables were significant. The final model B equation was:

Total Water Intake $=2,921.48$-342.88 Mexican American - 409.42 Other Hispanic - 193.18 Non-Hispanic White - 754.78 NonHispanic Black +0.75 Kcals - 2.94 Age +22.29 Height + 19.83BMI - 17.51 Osmolality. 
Table 23. Final model B of estimated coefficients and significance without gender

\begin{tabular}{|c|c|c|c|c|c|c|}
\hline \multirow[b]{2}{*}{ Variable } & \multirow[b]{2}{*}{ Coefficient } & \multirow[b]{2}{*}{$S E$} & \multicolumn{2}{|c|}{$95 \% \mathrm{CI}$} & \multirow[b]{2}{*}{$t$} & \multirow[b]{2}{*}{$p$-value } \\
\hline & & & Lower & Upper & & \\
\hline \multicolumn{7}{|l|}{ Race/ethnicity } \\
\hline Mexican American & -342.88 & 106.46 & -557.43 & -128.32 & -3.22 & 0.002 \\
\hline Other Hispanic & -409.42 & 130.56 & -672.55 & -146.30 & -3.14 & 0.003 \\
\hline Non-Hispanic White & -193.18 & 100.24 & -395.19 & 8.83 & -1.93 & 0.060 \\
\hline Non-Hispanic Black & -754.78 & 103.82 & -964.02 & -545.54 & -7.27 & $<0.0001$ \\
\hline Multi-Racial/Other & - & - & - & - & - & - \\
\hline Kcals & 0.75 & 0.02 & 0.71 & 0.80 & 31.26 & $<0.0001$ \\
\hline Age & -2.94 & 1.24 & -5.44 & -0.45 & -2.38 & 0.02 \\
\hline Height & 22.29 & 1.51 & 19.24 & 25.34 & 14.74 & $<0.0001$ \\
\hline BMI & 19.83 & 3.01 & 13.76 & 25.90 & 6.59 & $<0.0001$ \\
\hline Osmolality & -17.51 & 5.03 & -27.64 & -7.38 & -3.48 & 0.001 \\
\hline
\end{tabular}

Note: Bolded variables were statistically significant at $p<0.05$.

$\underline{\text { Summary of specific aim } 4}$

Significant predictors in final model A included race/ethnicity, kcals, age, height, FFM, and gender. Significant predictors in final model B included race/ethnicity, kcals, age, height, BMI, and osmolality. Final model A (with FFM) had an $r^{2}$ of $27 \%$ and a RMSE of 1,627.56. Final model B (without FFM) had an $r^{2}$ of $28 \%$ and a RMSE of 1,473.84. The F statistic was higher in model B without FFM compared to model A with FFM, 166.12 vs. 111.27, respectively. Model A did not include BMI and osmolality but did include gender while model B included BMI and osmolality but did not include gender. Six predictor variables were retained in final model A and six were retained in final model B. 


\section{$\underline{\text { Final prediction equations }}$}

The final prediction equation for model A was:

Total Water Intake $=-721.87-450.73$ Mexican American - 622.27 Other Hispanic - 320.45 Non-Hispanic White - 1,021.67 Non-Hispanic Black +0.78 Kcals +10.24 Age +11.00 Height +17.93 FFM - 176.37 Male.

The final prediction equation for model B was:

Total Water Intake $=2,921.48$-342.88 Mexican American - 409.42 Other Hispanic - 193.18 Non-Hispanic White - 754.78 NonHispanic Black +0.75 Kcals -2.94 Age +22.29 Height + 19.83 BMI - 17.51 Osmolality. 


\section{CHAPTER V.}

\section{DISCUSSION}

\section{Overview}

An improvement in the accuracy of estimating water requirements would immensely impact the field of nutrition. Registered dietitians (RDs) routinely assess the nutrient intake of individuals, but the estimation of the water requirements has been confusing and lacking in evidence-based research (ADA, 2007). This leads to uncertainty in the public regarding how much water is necessary to meet water requirements (Valtin, 2002). Equations which can accurately assess water requirements would improve the care of all individuals.

Water requirement equations that are more accurate also have public health implications. The current Dietary Guidelines for Americans do not specify water intake goals (USDHHS \& USDA, 2010). If the 2015 version of the Dietary Guidelines for Americans includes information for water intake requirements there would be an improved understanding of water intake requirements.

It would also be suitable for water intake recommendations to the public to vary based on factors that are known to affect water intake, such as age or FFM. Educational opportunities could be provided to caregivers in order to improve the health and wellbeing of the individuals under their charge. Federally funded programs would be able to apply these enhanced guidelines for the planning and implementation of programs specific to the needy.

There has been an increased prevalence of obesity (Ogden, Carroll, McDowell, \& Flegal, 2007) which has an immense impact on public health. There are numerous dietary 
myths regarding the adequate amount of water intake for those needing to lose weight (Valtin, 2002). An improvement in the knowledge of how much water is required for the overweight and obese can aid in treatment plans.

Presently there is a lack of evidence regarding the amount of total water to provide individuals living in institutional settings. Individuals in these facilities i.e., nursing homes, long term care facilities, psychiatric facilities, and prison, are currently supplied with an estimate of their water requirements based on equations which have not been validated. Information about water requirements for individuals in these types of settings would have enormous repercussions for all healthcare providers by improving the outcomes to those individuals housed in institutionalized setting.

An evidence-based equation would ultimately augment the current knowledge for the nutrient requirement of water. This type of information could advance the science of nutrition and add to public awareness regarding the appropriate amount of water intake, ultimately improving health-related outcomes.

In reviewing the results of this study, there were a number of significant findings. First, there were significant differences between the EWR equations indicating that they did not provide similar values for EWR. Thus, assessment of water requirements based on any one of the weight-based or energy-based EWR equations would not agree with an assessment determined by any of the other weight-based or energy-based EWR equations.

Secondly, when TWI was considered as an estimate of water requirements in this study, there was a lack of agreement between TWI and the values calculated from the EWR equations. That is, the actual water intake of a representative sample of the U.S. 
population was not adequately explained by the EWR equations. Therefore, it was not possible to predict water requirements using these EWR equations.

Third, in this study Fat-free mass (FFM) was found to have a weak positive association to TWI. This association was the strongest in the lowest BMI category. In underweight subjects FFM and TWI had a slightly higher correlation than in normal weight, overweight, and obese subjects. Since there was a positive association between FFM and TWI, albeit weak in all BMI categories, there was justification to include FFM as a credible predictor of TWI.

Lastly, neither of the models (models A with FFM or model B without FFM) was able to accurately predict TWI. Both final models contained six variables. One model (model A) contained FFM as a predictor of TWI, with the final model containing race/ethnicity, kcal intake, age, height, and gender as predictors of TWI. The other model (model B) did not include FFM as a predictor of TWI; this final model contained race/ethnicity, kcal intake, age height, BMI, and osmolality as predictors of TWI. Although these results have brought further insight into issues associated with water requirements, some methodological considerations must be recognized. These results suggest that TWI may not be an accurate method by which to predict water requirements. The main assumption in using TWI to predict water requirements is that regulatory mechanisms will drive individuals to consume sufficient TWI to meet their water requirements. The IOM considers this to be a valid and well-established assumption (IOM, 2005a). However, this assumption addresses only the physiological drive to consume adequate water to meet minimum needs. There is no equivalent regulatory mechanism to prevent individuals from consuming considerably more than 
their water requirements. The data from this study suggest that a significant number of individuals may have consumed more or less water than their water requirement, limiting the conclusions that can be drawn from these findings. It is important to explore more sensitive techniques to obtain estimates of water requirements. The remainder of the discussion chapter will prove a deeper examination of these issues.

\section{NHANES data}

Before the results of this study are discussed, it is beneficial to review the merits of the NHANES dataset to answer these types of research questions. The sample size in this dataset was much larger than would be available in most experimental studies, which held numerous advantages. It is recommended by the CDC that two or more (2-year) cycles be combined and analyzed in order to: 1) increase the sample size, and 2) decrease the likelihood of making a type 1 error (CDC, 2009). A confounding variable that could not be controlled for in this study was the environmental conditions. In an attempt to offset the variability from environmental differences, the largest sample possible from across the country and during various seasons was included in this study. Combining three cycles, i.e., six years of data from the NHANES dataset, increased the statistical reliability of the analysis.

Having a large sample size also made it possible to divide the sample into numerous smaller groups for comparisons while still maintaining a large enough sample size (sufficient power) to show significance. The data was a representative sample of the U.S. population and in that sense the interpretation of the results could be easily generalized to the civilian population not residing in an institutional setting. 
The NHANES dataset afforded diversity in the data that may otherwise not have been available to study. Included in the sample were individuals from different age groups, race/ethnicities, body weights, heights, etc. Since this sample was comprised of all individuals who were non-institutionalized and civilian (CDC, 2010f), there was an ability to include healthy as well as sick individuals in the sample.

There are numerous other benefits of using the NHANES dataset. The data collection methods employed by NHANES are well-documented and the data are collected in a very stringent manner using strict protocols (CDC, 2010d). The accuracy of the data collection and of quality assurance was also standardized (CDC, 2010d). It is also helpful that these data were readily and easily available on-line (CDC, 2010a; CDC, 2010b; CDC, 2010c).

\section{Aim 1 - agreement among EWR equations}

Because none of the five equations commonly used in clinical practice agreed with each other, it is unclear if any of them would provide a good estimate of an individual's water requirement. The fact that these equations did not agree with each other means that there is a discrepancy in the amount of water that would be recommended by RDs using different EWR equations in their specific care settings. Unfortunately, there is no actual standard for EWR equations; different equations are being utilized between care settings and perhaps between RDs within one care setting.

The criterion for agreement was not met by the two weight-based equations. The two weight-based equations i.e., the Linear equation and the Adjusted equation did meet the first criterion for agreement by having a very high correlation coefficient $(r=0.999, p$ 
$<0.0001$ ) (Table 6). Since both of these equations contained the sole variable of weight in their formulation, it is not surprising that there was a high association between them. Comparisons made between two variables that are similar to each other, or in this case the same variable, are very likely to be highly correlated with high correlation coefficients (Bland \& Altman, 1986).

The second criterion for agreement was that the line generated from the comparison of the equations to each other could not be significantly different from the line of equality. This technique, performed in other similar studies (Claros et al., 2005; Huang et al., 2002; Huang et al., 2003; Volaufova, 2005), consisted of building the $95 \%$ CI for both the slope and the intercept of a linear regression equation. When the Linear and Adjusted equations were compared to the line of equality, the $95 \% \mathrm{CI}$ of the slope and the intercept did not include one or zero, respectively (Table $5 \mathrm{a}$ and $5 \mathrm{~b}$ and Figure 2). Without the possibility that the slope was one or that the intercept was zero, the linear versus adjusted regression line could not be equal to the $\mathrm{x}=\mathrm{y}$ line of equality. Thus, the weight-based equations did not agree with each other.

It is helpful to keep in mind that correlations are just a measure of linear association, which do not change no matter which variable comes first. In other words, the same correlation coefficient would be derived regardless of which variable was independent and which was dependent, i.e. which variable was on the $\mathrm{X}$ axis or on the $\mathrm{Y}$ axis. The degree of correlation was related to the slope of the linear relationship. On the other hand, the form of the linear relationship would change depending on which variable came first as the dependent variable and which came after as the independent variable. When the independent variable was equal to zero, the dependent variable was equal to the 
intercept. This intercept was the value in which the dependent variable started in its linear relationship with the independent variable. Thus, the value and magnitude of the intercept was very important to the lack of agreement for these equations. In order to obtain an intercept of zero, the slopes of both variables had to be one and their means had to be equal to each other.

When compared to each other, the energy-based equations (i.e. the Harris, Mifflin, and NRC equations) did not meet both criteria for agreement either. Similar to the weight-based equations, the energy-based equations had very high correlation coefficients when compared to one another (Table 6); the Harris versus Mifflin $(r=$ $0.986)$, the Mifflin versus NRC $(r=0.977)$, and the Harris versus NRC $(r=0.978)$ were considered very strongly associated bivariate pairings (Overholser \& Sowinski, 2008). The Harris versus Mifflin equations appeared to agree with each other (Figure 9). Despite the appearance of similarity to one another and the similarity in the equations, even with the large sample size of 10,468 , these two equations did not meet the criteria for agreement. It is not surprising that these energy-based equations were highly associated with each other because, like many energy expenditure equations, they contained similar variables (age, height, weight, and gender) in their equation (Harris \& Benedict, 1919; Mifflin et al., 1990; IOM, 2005b). The NRC equation also contained a physical activity (PA) factor. However, this PA is eliminated as a factor when the eligibility criteria excluded those individuals with self-reported PA level of "very active".

Even though the energy-based equations were highly correlated to each other, they lacked agreement, i.e., all three of the bivariate pairings were significantly different from the line of equality (Table $5 \mathrm{a}$ and $5 \mathrm{~b}$ and Figures 9-11). Although these equations 
had the same or similar variables that were highly correlated, they were different enough from each other when tested in a large dataset to say that they did not agree in their values.

When the weight-based equations were compared to the energy-based equations, they had high correlation coefficients (Table 6). The Linear and Harris $(r=0.869)$, Adjusted and Harris $(r=0.869)$, Linear and Mifflin $(r=0.857)$, Adjusted and Mifflin $(r=$ 0.859), Linear and NRC $(r=0.769)$, and Adjusted and NRC $(r=0.770)$ were all highly associated to each other. The likely reason for the high correlations was that all of these equations contained the variable of body weight.

Overall, the weight-based equations were less correlated to the energy-based equations than were the weight-based equations to each other or the energy-based equations to each other. This is likely because there were variables other than weight in the energy-based equations, i.e., age, gender, and height, which may have modified the relationship between weight-based and energy-based equations.

Age is an example of a variable that may modify the amount of TWI between individuals. It has been shown in the literature that older individuals consume less water than younger individuals (Kant et al., 2009; Phillips et al., 1984; Phillips et al., 1991; Zizza et al., 2009). Decreased water intake with age may occur due to a decrease in FFM with age (Baumgartner, 2000; Feinsod et al., 2004; Ferry, 2005; Houston et al., 2009; Luckey \& Parsa, 2003; Rivlin, 2007). In a representative sample of the US population, Kant et al. (2009) concluded that with more advanced age (20-39, 40-59, $\geq 60$ yrs), individuals ingested significantly less total water intake (mean of 20-39 $\mathrm{yrs}=3,300 \mathrm{ml} \pm$ 118 , mean of 40-59 yrs $=3,390 \mathrm{ml} \pm 70$, mean of $\geq 60 \mathrm{yrs}=2,650 \mathrm{ml} \pm 65 ; p<0.0001)$. 
These investigators did not include metabolic water as part of total water intake as was done in the present study. Other past research (Phillips et al., 1984; Phillips et al., 1991) demonstrated a decrease in the thirst drive of older adults resulting in less water intake than in younger individuals. In a representative sample of the U.S. population, Zizza et al. (2009) reported that people in the two oldest age groups consumed less total water intake than those in the youngest age group $(65-75 \mathrm{yrs}$ mean $=2905 \mathrm{ml}, 75-84 \mathrm{yrs}$ mean $=2573$ $\mathrm{ml}, \geq 85$ yrs mean $=2275 \mathrm{ml} ; p<0.05)$.

The gender variable may also have modified the relationship between the weightbased and energy-based equations due to known differences in the FFM between genders (Zamboni et al., 2003). Men consume more total water than women, and these differences between men and women are consistent with the literature (Bossingham et al., 2005; Kant et al., 2009; Raman et al., 2004). In a representative sample of the U.S. population, Kant et al. reported that men ingested significantly more total water intake than women (mean of men $=3467 \mathrm{ml} \pm 79$ mean of women $=2897 \mathrm{ml} \pm 72 ; p<0.0001)$. In a large water turnover study conducted in 458 adults, Raman et al. (2004) reported total water intake of individuals aged $40-79 \mathrm{yrs}$ as $3.0 \mathrm{l} /$ day in men (range: $1.4-7.7 \mathrm{l} /$ day) and $2.5 \mathrm{l} /$ day in women (range: 1.2-4.6 1/day). These investigators however, only performed statistical analysis within each gender group not between genders. Bossingham et al. (2005) reported that younger $(n=11 ; 4,150 \mathrm{ml} \pm 804)$ and older men $(n=10 ; 3,996 \mathrm{ml} \pm 943)$ ingested more total water than younger $(n=14 ; 3,451 \mathrm{ml} \pm 744)$ and older women $(n=11 ; 3498 \mathrm{ml} \pm 337)$ within the same age groups $(p<0.05)$.

Additionally BMI, which is an index created from the height and weight variable, may have modified the relationship between the weight-based and energy-based 
equations if individuals with higher BMI values consumed more water as discussed in the literature (Kant et al., 2009). This may have occurred if individuals with higher BMI values lost more water as a result of larger losses as insensible respiratory losses. Individuals with higher BMI values may also have larger transcutaneous losses than those with lower BMI values due to a larger body surface area.

Since the weight-based equations lacked agreement with each other and the energy-based equations lacked agreement with each other, it is not surprising that the weight-based equations lacked agreement with the energy-based equations. All six of the bivariate pairings between weight-based and energy-based equations were significantly different from the line of equality (Table 5a and 5b and Figures 3-8). Therefore although the weight-based equations contained similar enough information to be highly associated with each other, they were significantly different from each other in value.

In conclusion, it is very likely that RDs in clinical practice and across care settings are recommending different amounts of water to individuals depending on which equation they are selecting. The goal of the NCP is standardization of care to all client/patients in order to provide the optimal outcomes (IDNT, 2009). It is unlikely that there is standardization of care for the nutrient water given the differences in values discovered in this current study.

\section{$\underline{\text { Aim } 2 \text { - agreement between TWI and EWR equations }}$}

This study revealed little or no agreement between water requirements predicted by the EWR equations and TWI. The largest $r^{2}$ of 0.081 occurred when TWI was regressed on the NRC equation. This meant that only $8.1 \%$ of the variability of TWI 
could be explained by the NRC equation. A very small amount of the variability of TWI was explained by the independent variable of NRC. The best model of the five equations (NRC equation) was a poor predictor of TWI.

The first criterion for agreement was not met. Although all regressions and correlations involving the TWI variable were statistically significant at the $p<0.0001$ level, there were only weak bivariate associations. Because of the weak association demonstrated, further testing to compare the regression line to the line of equality would not provide additional valuable information regarding agreement between TWI and each of the five EWR equations.

This finding may be due to limitations in the use of TWI as an estimate of water requirements. It may be the case that some people were consuming significantly more or less water than was required to replace physiological losses.

Total water intake was accepted as an estimate of water requirements based on well-documented assumptions regarding physiology and the thirst mechanism (IOM, 2005a). The IOM had stated that individuals will consume, within a reasonable amount of time, the total water needed to compensate for losses based on the thirst mechanism. The method of using TWI as an estimate of water requirements was selected based on the validity of this assumption.

While it is thirst that is responsible for the amount of water intake this is only true for meeting the minimum water requirement (Guyton \& Hall, 2006). The other assumption in using TWI as an estimate of water requirements is that individuals would not consume significantly more water than they required. However, individuals in this study may have consumed substantially more than needed. Unlike the thirst mechanism 
to maintain a minimum water intake, there is no equivalent regulatory process or mechanism in the body to limit consumption of water beyond physiological need. If water consumption was in excess of water requirements, it would explain why TWI was not in agreement with any of the equations.

The body does have a mechanism to rid itself of water beyond that which is needed, i.e., amount of water larger than that needed to excrete the renal solute load (Manz et al., 2002). This is called the free water reserve. Most of the free water reserve will leave the body as urine. However data on urine output was not available from NHANES. One of the limitations in this study was the lack of data for urine output to determine the crude amount of water output. An analysis of the volume of urine output could have allowed a determination of the free water reserve.

There are many behavioral and social factors which may cause individuals to consume water above physiological needs; these factors include the increased trend in the consumption of bottled water and appealing beverages (Doria, 2006; Nielsen \& Popkin, 2004). There has been an explosion of bottled water consumption in recent years (Lappalainen et al., 1993). This bottled water phenomenon was most likely the result of a misconception of facts regarding water safety from the tap as well as the quantity of water needed for health purposes. The portability of bottled water may also have made its increased ingestion more likely. However, the need to drink eight glasses of water per day is not evidence-based and has not been proven to be the water requirement (Valtin, 2002).

Besides drinking plain water to quench thirst, other tempting sources of water consumption have gained popularity as a socially acceptable source of water intake in the 
last quarter century (Nielsen \& Popkin, 2004). Sugar-sweetened beverage consumption has more than doubled in the last 50 years (Popkin, 2010). Re-invented or re-packaged and appealing sources of water such as caffeinated beverages (from specialty coffee houses) and alcoholic beverages (from martini bars) have also increased over the last 20 years (Nielsen \& Popkin, 2004).

Other misconceptions regarding the amount of water intake and the role of water in health and disease may have contributed to increased water intake. For example, one study concluded that drinking extra water, especially if the water was heated, could aid in weight loss because it increased metabolism by $30 \%$ within ten minutes of ingestion (Boschmann, Steiniger, Hille, Tank, Adams, Sharma, Klaus, Luft, \& Jordan, 2003). Individuals who believed this to be true might have ingested large amounts of water hoping to lose weight.

It may be better to estimate water requirements using a measure (body weight) rather than another estimate (estimated energy requirements). In clinical practice it is not feasible to measure energy requirements directly; they are always estimated by equations derived from small populations. Body weight, on the other hand, is a direct measure of body mass, not an estimate.

\section{Aim 3 - correlation of FFM and TWI}

Fat-free mass was weakly and positively correlated with TWI at all categories of BMI $(n=4,422)$. These correlations were all significant $(p<0.0001)$. The strongest association to TWI was in the underweight category (BMI $<18.5)$ with $r=0.327$. The weakest correlation was in the obese category (BMI $\geq 30)$ with $r=0.243$. 
Although the strongest correlation was slightly greater than 0.3 for the lowest BMI group (BMI < 18.5), this is considered a weak correlation (Overholser \& Sowinsi, 2008). The other three BMI groups had correlation coefficients less than 0.3 indicating a relationship but one that is also considered weak (Overholser \& Sowinsi, 2008).

There is a certain proportion of lean to fat tissue assumed when BMI is utilized as a measure of adiposity (Hammond, 2004). Because BMI is simply the ratio of weight to height, it is expected that in normal-weight individuals, certain weight ranges will be associated with certain height ranges so that the proportion of lean to fat tissue is within normal limits. Using this assumption, most of the weight in underweight individuals would come from FFM and only a small amount would come from fat tissue.

The highest correlation of FFM to TWI was in the underweight category. If the largest portion of these individuals was from lean tissue, and lean tissue was positively associated with water requirements, it is not surprising that these individuals would show the highest correlation to TWI. There are also a relatively larger proportion of organs in underweight individuals. Organs are the most metabolically active lean tissue and would therefore likely be associated with the largest amount of water. Lastly, the underweight category of BMI had the smallest sample size (4.4\%). If the sample size was greater, this association might have been stronger or weaker.

It was surprising that the overweight and obese individuals did not have a higher association with TWI. It takes more muscle (lean tissue) to move a bigger body and all individuals examined at the MEC were ambulatory (Gropper, Smith, \& Groff, 2009). It would therefore be expected that overweight or obese individuals would have a higher absolute amount of FFM. Yet the correlation of FFM in overweight and obese individuals 
was weak. This finding likely reflects the variability in body composition as individuals gain weight.

A positive association only demonstrates that TWI increased as FFM increased. Not all participants were given the BIA examination, but there was enough data from those individuals who underwent BIA to determine that there was a statistically significant, albeit weak, association between FFM and TWI in all BMI categories. Thus, there was justification to include FFM as a credible predictor of TWI under Specific Aim 4.

The biggest limitation in this analysis was that only those aged 19-49 were given BIA assessment of FFM. Therefore, any conclusion drawn from these results must reflect this age limitation. The CDC did not give an explanation of why individuals $>49$ did not have BIA assessment, but it was not uncommon for questions from the questionnaire, laboratory values from the laboratory examination, or tests from the examination portion of the MEC examination to be changed between two-year cycles without explanation.

Another limitation in this analysis was that it was assumed that those individuals who received BIA had fasted for at least six hours. This protocol was created because those individuals who were receiving a fasting blood draw were also slated to undergo the BIA procedure (CDC, 2010a - 2010c). However, after the blood draw, these individual may or may not have been offered and ingested a snack. Therefore, there may have been a difference in the BIA readings depending on whether they had fasted or consumed a snack. Further, there is no way to determine which individuals had consumed a snack. 


\section{Aim 4 - model building to predict TWI}

In order to address specific aim 4, model A with FFM and model B without FFM were created. These models were important to formulate and evaluate because this was a representative sample of the civilian non-institutionalized population. These models could be a beneficial tool to gather further insight into the variables that predict TWI in this representative sample.

Final models A and B, each containing six variables, were created to explain the TWI of individuals. Both models started with all of the variables of interest from the literature as described below or of known clinical or biological importance.

As a highly hydrated tissue in the body, FFM was a biologically relevant variable to consider in one of the created models (model A) (Wang et al., 1999). The univariate analysis in the unweighted data $(n=4,480)$ produced a large significance level for FFM $(p<0.0001)$ which was instrumental in the decision to include it in this model. If FFM was shown to be an important predictor of water requirements, RDs might be able to improve the accuracy of water requirement assessment with a portable and easy-to-use measure of FFM like BIA.

Both full models to predict TWI also included age. The univariate analysis $(n=$ 10,732) produced a large significance level for age $(p<0.0001)$. Kant et al. $(2009)$ and Zizza et al. (2009) both found that older adults consumed less TWI than younger adults. Bossingham et al., 2005 reported that water intake from food, beverages, and metabolic water was higher in younger men and women than in older men and women (Bossingham et al., 2005). These findings in the literature were influential in the decision to include age in the full model. 
BMI was included in both full model A and full model B. The univariate analysis of the unweighted data $(n=10,470)$ produced a large significance level for BMI $(p=$ 0.0024). Past research determined that BMI differences affect TWI. Kant et al., 2009 reported that as energy-adjusted BMI increased, individuals consumed more TWI. Those investigators found that individuals with a BMI $<25 \mathrm{~kg} / \mathrm{m}^{2}$ had the lowest TWI $(2,967 \mathrm{ml}$ $\pm 78 ; p=0.001)$, individuals with a BMI of $25-<30 \mathrm{~kg} / \mathrm{m}^{2}$ had higher TWI $(3,190 \mathrm{ml} \pm 85$; $p=0.001)$ and individuals in the highest BMI category of $\geq 30 \mathrm{~kg} / \mathrm{m}^{2}$ had the highest TWI $(3,375 \mathrm{ml} \pm 89 ; p=0.001)$. The implication was that individuals with a higher BMI might have consumed more water than those with a lower BMI, regardless of the total amount of FFM.

Both full models to predict TWI also included kcals ingested. The univariate analysis of the unweighted data $(n=10,732)$ produced a large significance level for kcals ingested $(p<0.0001)$. There was evidence from the literature demonstrating that the majority of TWI comes from the kcals ingested in food and non-water beverages (IOM, 2005a; Kant et al., 2009).

The full models that predicted TWI also included weight $(n=10,546)$, height $(n=$ $10,535)$ and gender $(n=10,732)$. The univariate analysis of the unweighted data for the continuous variables of weight and height variables produced a large significance level for both $(p<0.0001)$. These variables along with age are predictors for most energy expenditure equations (Frankenfield et al., 2005; Mifflin et al., 1990). Energy expenditure equations were the foundation for the energy-based water requirement equations that were tested in this study. Adolph (1933) stated that energy expenditure was linked to water requirements. Adolph was the first researcher to suggest that $1 \mathrm{ml} / \mathrm{kcal}$ expended 
would be a convenient standard for water requirements. All of the variables common to energy expenditure equations (including weight, height, and gender) were therefore justified to be included in the model.

Full model A and B also included osmolality. The univariate analysis of the unweighted data $(n=10,147)$ produced a large significance level for osmolality ( $p$ $<0.0001)$. There was an established relationship between osmolality and water in the literature (Bayliss \& Thompson, 1988; Martin et al., 2006). The IOM considers osmolality as the criterion of adequacy when determining the hydration status of individuals because increased osmolality causes the physiological mechanism of thirst to initiate drinking and consequently increases TWI (IOM, 2005a).

The full model A and the full model B to predict TWI also included race/ethnicity $(n=10,732)$. There was evidence in the literature demonstrating the difference in TWI between ethnic and racial groups (Kant et al., 2009). Kant et al. (2009) reported differences in TWI between Non-Hispanic Whites $(3,327 \mathrm{ml} \pm 80)$, Non-Hispanic Blacks $(2,636 \mathrm{ml} \pm 86)$, and Mexican-Americans $(2,877 \mathrm{ml} \pm 107 ; p<0.0001)$. The disparities seen in this study between race/ethnicities may be an important piece of information regarding future assessment of water requirements of different race/ethnicities. It was therefore important to include this variable in both full models.

Both models A and B started with the eight variables of race/ethnicity, kcals, age, height, weight, BMI, osmolality and gender in the full model. Model A also included FFM as the ninth variable in the full model. 


\section{Model A (with fat-free mass)}

Full model A with the nine predictor variables including FFM explained 27\% of the variability in TWI. When the VIF was calculated, body weight returned the highest value $(\mathrm{VIF}=113.64)$. This meant that body weight was highly correlated with the other variables in the model. The body weight variable brought some of the same information to the model as BMI (which contains weight), kcals, height, FFM, and gender. Due to this high VIF, body weight was taken out of the model first. The fact that body weight contained the highest VIF is not surprising since it is often the sole predictor of water requirements (Akner \& Floistrup, 2003; Biesalski et al., 2009; Chernoff, 1994b; Chidester \& Spangler, 1997; Grant \& DeHoog, 1999; Holben et al., 1999; Juan \& Basiotis, 2002; Lipp et al., 1999; Wotton, et al., 2008).

Model A, without the body weight variable, still explained $27 \%$ of the variability in TWI. The VIF was rechecked and none of the variables contained an excessive amount of multicollinearity (VIF $>10)$. Five of the remaining variables were significant in the second regression of model A. These variables were race/ethnicity $(p<0.0001)$, kcals $(p<0.0001)$, age $(p=0.017)$, height $(p=0.046)$, and FFM $(p=0.005)$. Checking the coefficients for changes greater than $20-25 \%$ was not performed since removal of the weight variable also removed the remaining multicollinearity problems. The large change in coefficients and $p$-values was to be expected.

This second regression model A without the weight variable contained several variables with $p$-values $>0.05$. The highest $p$-value was for BMI $(p=0.916)$. Thus, BMI was the next variable to be removed from model A. 
The third regression model A did not contain BMI but did contain FFM. Since FFM was included in the model, BMI may not have brought anything new to model A that was not more exclusively addressed by the FFM variable (Hammond, 2004). In fact it is the fat-free tissue that is highly hydrated (Wang et al., 1999). It therefore makes sense that the tissue that is highly associated with water would be a better predictor of TWI than a measure of adiposity such as BMI when FFM was in the model.

It must be remembered that FFM does not stay the same throughout the life cycle. The sample in this study only included those individuals age 19-49. But individuals lose FFM as they age (Baumgartner, 2000; Kyle et al., 2001; Shaw, Srikanth, Fryer, Blizzard, Dwyer, \& Venn, 2007). Since this sample did not contain individuals age 50 and older, the amount of FFM may not have been modified by age-related changes as would occur if the entire range of ages were available.

Without the BMI variable, the third regression of model A still explained $27 \%$ of the variability in TWI. Thus, taking out BMI did not appear to change the strength of model A to predict TWI. The VIF was rechecked and none of the variables contained an excessive amount of multicollinearity (VIF $>10)$. Five of the remaining variables were significant in the third regression model A. These variables were race/ethnicity $(p<0.0001)$, kcals $(p<0.0001)$, age $(p=0.019)$, height $(p=0.009)$, and FFM $(p<0.0001)$. Evidence of confounding was then checked. All of the coefficients, before and after removal of the BMI variable were checked for a $20-25 \%$ change in value and for a difference in significance. None of the variables showed evidence of confounding. 
The third regression model A without the BMI variable contained several variables with $p$-values $>0.05$. The highest $p$-value was for osmolality $(p=0.530)$. Thus, osmolality was the next variable to be removed from model A.

A possible explanation for osmolality to be non-significant and therefore dropped from the model was directly related to the age range of model A; the sample was relatively young (19-49 yrs). Therefore, osmolality differences within this age range would not be expected. On the other hand, osmolality differences would be expected from older individuals. The range of osmolality in this study for all ages was 241-310 mOsm/L. Phillips et al. (1984) reported that healthy older men who were water deprived had higher plasma osmolality levels than younger men. When men were infused with saline, both older men and younger men had an increased osmolality, however only the older men were unable to replace the amount of total water needed to reduce the osmolality back to normal levels (Phillips et al., 1991).

Older individuals, who were not included in model A, have an attenuated thirst mechanism compared to younger adults (Ferry, 2005; Grandjean \& Campbell, 2004; Jecquier \& Constant, 2010; Kavouras, 2002; Stachenfeld, Mack, Takamata, DiPietro, \& Nadel, 1996). Older individuals are more likely to be on medications which induce thirst, independent of natural physiological changes (Luckey \& Parsa, 2003; Thomas et al., 2008). Additionally, older adults are more likely than younger adults to be put on diuretic medication due to increased incidence of hypertension (Rosenthal, Shamiss, \& Holtzman, 2001). Diuretic medications are meant to decrease hypertension by increasing water losses in the urine (Abdallah et al., 2009; Berl, 2008; Louden, 2009). Therefore the eligibility criteria in this study excluded individuals on diuretic medications. 
The functionality of the kidney has also been shown to be decreased in older versus younger adults (Berl, 2008; Chernoff, 1994a; Grandjean \& Campbell, 2004; Jecquier \& Constant, 2010; Kayser-Jones et al., 1999). This may cause osmolality differences with age. Other age-related kidney function changes can occur that can alter the osmolality of older adults. These changes could not be discerned in this fourth model A due to the age range (19-49 years).

With increasing age individuals are also more likely to become insulin resistant and prone to pre-diabetes and type 2 diabetes (CDC, 2009; Neiheisel, Sudduth, \& Schmidt Luggen, 2010). Individuals with higher blood glucose levels would likely have increased plasma osmolality. Since model A used the variable of FFM which was only performed on individuals age 19-49 yrs, it is difficult to determine if osmolality would have been retained in model A if older individuals were included as well.

The fourth regression model A, without the osmolality variable but with the remaining six predictor variables, continued to explain $27 \%$ of the variability in TWI. Thus, taking out osmolality did not appear to change the strength of model A to predict TWI. The VIF was rechecked and none of the variables contained an excessive amount of multicollinearity (VIF $>10)$. Six of the remaining variables were significant in the fourth model A. These variables were race/ethnicity $(p<0.0001)$, kcals $(p<0.0001)$, age $(p=$ $0.014)$, height $(p=0.013)$, FFM $(p<0.0001)$, and gender $(p=0.048)$. None of the remaining variables showed evidence of confounding.

The fourth regression model A without the osmolality variable contained only significant variables with $p$-values $<0.05$. The six variables, FFM, race/ethnicity, kcals, age, height, and gender, were retained in the final model. 
The retention of these six variables in final model A can be explained by what is known about their relationship to water as well as other studies found in the literature. The FFM retention in the model does not come as a surprise, since most body water is located within the FFM (Ritz \& Berrut, 2005). Age seemed to modify the hydration constant of the FFM. Bossingham et al. (2005) found that older adults versus younger adults had less FFM and higher total body water (TBW) to FFM ratio. Older adults were more likely than younger adults to be less physical active, have sarcopenia, and have chronic debilitating illness and disabilities (Houston et al., 2009). A big limitation of this study was the fact that the model that included FFM only included those individuals up to age 49. This is likely the reason that age had a positive coefficient in model A rather than a negative one. It is possible that if the full age range was included there might be a negative coefficient in the model because as individuals age, TWI decreases (Jecquier \& Constant, 2010). It is also possible that there would be a positive coefficient of age until age 49 and then a negative coefficient thereafter.

BMI was not retained in final model A. Raman et al. (2004) reported that BMI was not positively associated with a higher TWI in free-living adults. This suggested that if water requirements were based on TWI, body size did not influence water requirements. However, it was suspected that if FFM was not in the final model, BMI would have more influence on the variables especially without the weight variable in model A. Fat-free mass demonstrated a positive association with TWI in this study at all BMI categories but especially in the underweight category.

Kcal intake was retained as a predictor of TWI. The assumption here was that individuals who were in energy balance would consume their energy requirements and 
expend it. Many researchers utilize energy expenditure equations as a means to determine water requirements (Armstrong-Esther et al., 1996; Chidester \& Spangler, 1997; Feinsod et al., 2004; Ferry, 2005; FNB, 1989; Kayser-Jones et al., 1999). This is done by using the equation $1 \mathrm{ml}$ of water required/kcal expended. It was concluded in this study that three of these energy expenditure equations (Harris, Mifflin, and NRC) did not agree with TWI. Therefore, the $1 \mathrm{ml}$ of water required/kcal expended equation does not seem to predict TWI fully (i.e. 100\%).

The Food and Nutrition Board in 1989 recommended water intake of $1 \mathrm{ml}$ of water per kcal consumed (FNB, 1989). Kant et al. (2009) calculated that $66 \%$ of TWI came from the kcals eaten in food and beverages. It seemed likely that those individuals representative of the population consumed more kcals than required. Kcals consumed was therefore an important predictor of water requirement in final model A. However, kcals consumed was not a practical variable to obtain as it involves taking a complete diet history of the patient and is time-consuming.

Gender was retained in final model A despite being borderline significant $(p=$ 0.048). In fact, gender could have been dropped as its $p$-value decreased with each newer model. However, gender differences in TWI have been demonstrated numerous times in the literature (Bossingham et al., 2005; Kant et al., 2009; Raman et al., 2004). These differences showed that men consistently ingested significantly more in TWI than women (Bossingham et al.; Kant et al., 2009; Raman et al., 2004). Thus, the significance of gender could not be overlooked.

Body composition is different for men and women and these changes become even more apparent with age (Zamboni et al., 2003). That is, as men get older, they have 
less testosterone and lose FFM at a quicker pace than younger men (Zamboni et al., 2003). Women are protected from losses of FFM, especially in the bones by estrogen but this protection is eliminated for post-menopausal women. The percent of FFM lost in older men is larger than the percent of FFM lost in older women (Zamboni et al., 2003). Therefore, it was suspected that if there was a wider range of age in model A, gender would have been more highly significant.

In this study, males consumed less water than females when FFM was in the model. This would imply that the difference in TWI between males and females was due to more than FFM differences, and when the variability associated with FFM was removed from gender, males required less TWI than females. However, again this was only a limited age group of the total sample and gender was at the border of being not statistically significant. It was expected that with a full age range while including FFM in the model, males would have a significantly higher water intake than females.

Race/ethnicity was retained in final model A as a predictor of TWI. Compared to multiracial individuals (reference group), Non-Hispanic Blacks had the lowest TWI while Non-Hispanic Whites had the highest TWI. Kant, et al. (2009) also found similar ethnic differences in total water intake although these investigators tested different ethnic groupings in their study. Different ethnic groupings were available for use in the NHANES datasets (CDC, 2010a - c).

The literature review revealed that race/ethnicity was not typically taken into consideration in equations that were related to water intake. The Mifflin equation did not specify which race was considered in its formulation and the Harris equation only used Non-Hispanic Whites in its formulation (Frankenfield et al., 2005). In another study, a 
new energy expenditure equation was created when it was found that African-American and European Americans had different resting energy expenditures from one another (Vander Weg et al., 2004).

Ethnic differences might be due to numerous factors. Individuals from diverse ethnic groups and cultures eat different foods and food groups. For example it has been shown that different ethnic groups consume different amounts of fruits and vegetables (Zhao, Ford, \& Modkad, 2008). Data from the USDA/ARS show that fruits and vegetables have a very high water content of between 80 and 95\% (USDA/ARS, 2002). Everything else staying the same, those cultures that eat more fruits and vegetables may be taking in more TWI than other ethnic groups. Of course the possibility exists that these ethnic groups that take in more water in fruits and vegetables may be consuming less water from beverages.

Behavioral differences including willingness to adopt a healthy lifestyle were also shown between ethnic groups (Zhao et al., 2008). Consuming more fruits and vegetables are part of behaviors (such as increasing exercise and quitting smoking) which constitute the willingness to adopt a healthy lifestyle. Popkin et al. (2005) reported ethnic differences in water consumption between Mexican Americans who had a healthier dietary pattern (i.e., drinking more water, consuming more fruits and vegetables, and ingesting more low-fat dairy products) than Non-Hispanic Blacks in a representative sample of the U.S. population.

A recent investigation found that providing individuals with culturally inappropriate foods decreased food and beverage intake (Nieuwenhuizen, Weenen, Rigby, \& Hetherington, 2010). Nieuwenhuizen et al. (2010) only included older adults in 
that study. In the present study only younger adults were included but the concept of eating less when there are less acceptable foods available cannot be overlooked. Therefore, these factors may directly affect the TWI between ethnic groups.

\section{Model B (without fat-free mass)}

Full model B with the eight predictor variables of TWI not including FFM explained $28 \%$ of the variability in TWI. When the VIF was calculated, weight returned the highest value $(\mathrm{VIF}=90.91)$. This meant that like in model A, body weight was highly correlated with the other variables in model B and was therefore the first variable to be removed from model B.

The second regression model $\mathrm{B}$ without the body weight variable but with the remaining seven predictor variables again explained $28 \%$ of the variability in TWI. The VIF was rechecked and none of the variables contained an excessive amount of multicollinearity (VIF $>10)$. Six of the remaining variables were significant in the second regression model B. These variables were race/ethnicity $(p<0.0001)$, kcals $(p<0.0001)$, age $(p=0.020)$, height $(p<0.0001)$, BMI $(p<0.0001)$, and osmolality $(p=0.001)$. This second regression model B without the weight variable contained one variable with a $p$ values $>0.05$. The non-significant $p$-value was for gender $(p=0.98)$. Gender was the next variable to be removed from the model.

It was surprising that gender was the next variable removed from model B, since it was retained in model A. It has been shown in the literature that gender is an important predictor of TWI and that men consume more TWI than women (Kant et al., 2009; Zizza et al, 2009). Keep in mind that conditions under which Model A and Model B were built 
differ in two important ways: 1) the variable FFM was considered in the building of Model A but not in Model B; and 2) only 19-49 year olds were included in the sample for Model A and 19-85 year olds were included in the sample for Model B. If the sample in Model B, which included all ages $>19$ yrs, also contained the FFM variable, gender may have remained in the model. It seems that the accelerated decrease of FFM in men (Zamboni et al., 2003) more so than in women that normally occurs with aging may have rendered gender insignificant when the FFM variable was not included in the model. Age appeared to capture the variability in model B that was attributed to the gender variable in model A.

Another possible explanation is that osmolality may have modified the gender effect in this study. This study included osmolality in the model whereas other studies that looked at TWI did not include osmolality (Kant et al., 2009; Zizza et al., 2009). A study on the relationship between plasma arginine vasopressin and serum osmolality showed a significant age effect (higher in older subjects) but not a gender effect (Johnson, Crawford, Kelly, Nguyen, \& Gyory, 1994). Another study showed a greater increase in urine osmolality in males versus females with increased age (Manz \& Wentz, 2003). Thus, including the osmolality variable could have rendered gender nonsignificant.

The third regression model B without the gender variable but with the remaining six predictor variables again explained $28 \%$ of the variability in TWI. Thus, taking out gender did not appear to change the strength of model B to predict TWI. The VIF was rechecked and none of the variables contained an excessive amount of multicollinearity (VIF $>10)$. The six remaining variables were significant in the third regression model B. 
These variables were race/ethnicity $(p<0.0001)$, kcals $(p<0.0001)$, age $(p=0.020)$, height $(p<0.0001)$, BMI $(p<0.0001)$ and osmolality $(p=0.001)$. None of the variables showed evidence of confounding, contained only significant variables with $p$-values $<0.05$, and were therefore retained as final model B.

There was an age effect on TWI and it was retained in final model A and final model B. Age had a positive coefficient in final model A and a negative coefficient in final model B. As previously discussed, having FFM in the model without the full range of age is a limiting factor to fully explain these phenomena especially in model A. Having the full range of age in model B and a negative coefficient for age, however, is not surprising since it has been shown that individuals take in less TWI as they age (Jecquier \& Constant, 2010).

Ingesting less TWI may be due to a decreased thirst mechanism that occurs with age (Ferry, 2005; Grandjean \& Campbell, 2004; Jecquier \& Constant, 2010; Kavouras, 2002; Stachenfeld et al., 1996). Factors which would ordinarily increase thirst in younger individuals may be altered in older individuals. A decreased thirst mechanism would likely manifest as decreased TWI in older adults.

There appears to be a reduced thirst response in older adults despite a diminished ability to maximally concentrate the urine (Feinsod et al., 2004; Hodak \& Verbalis, 2005; Luckey \& Parsa, 2003; Schlanger et al., 2010). The result is greater water losses from the body without a physiological correction. Increased urine losses may be the result of an overall decline in kidney functioning with age (Chernoff, 1994a; Sansevero, 1997; Thomas et al., 2008). On the other hand, some investigators did not find differences in urine output between older and younger individuals (Bossingham et al., 2005; Raman et 
al., 2004). The altered thirst mechanism regardless of urine losses was likely responsible for differences in TWI between older and younger adults.

Older adults may suffer from illness, disabilities, or specific conditions which decrease the ability to ingest sufficient water in the same amounts as younger individuals (IOM, 2005a; Paterna et al., 2009). Cognitive changes which accompany increased age may also play a role in decreased TWI of older adults (Abdallah et al., 2009).

The osmolality coefficient was a negative coefficient in final model B, meaning those individuals with higher TWI had lower osmolality. This makes physiological sense (Bayliss \& Thompson, 1988; Guyton \& Hall, 2006; Jequier \& Constant, 2010). Investigators have shown higher plasma osmolality in older versus younger men secondary to changes in thirst with age (Phillips et al., 1984; Phillips et al., 1991). The association between osmolality and TWI was described in full in the model A section above.

In Model B, BMI seemed to function as a surrogate variable for FFM. Skeletal muscle mass is correlated to body mass which is why bigger individuals have more total FFM than smaller individuals (Gropper et al., 2009). Therefore, BMI is a limited but necessary substitute for FFM.

Race/ethnicity and Kcals ingested were also retained in final model B as they were in final model A. These variables have been shown to affect TWI and the rationale for their retention in model B is the same as was described previously in the discussion of final model A. 


\section{$\underline{\text { Water requirements using TWI as an estimate }}$}

The IOM explicitly stated that it was theoretically possible for TWI to be an estimate of water requirements (IOM, 2005a). The literature review revealed that this method, i.e., using TWI as an estimate of water requirements had never before been validated. Assuming that individuals would consume in TWI their water requirements and not substantially more or less, it would be possible to construct practical equations to predict TWI.

The results of specific aim 2 revealed that TWI did not agree with EWR equations currently used in clinical practice. However, the regression of TWI on each of the EWR equations contained a large amount of unexplained variability. The highest $r^{2}$ value was between NRC and TWI, but only approximately $8 \%$ of the variability of TWI was explained by the NRC equation. In the case of simple linear regression, the correlation coefficient is simply the square root of $r^{2}$. Low correlation coefficients, as is the case here, would stem from low $r^{2}$ values. It is assumed that the error associated with regression is related to the outcome variable (TWI).

In this study, there may have been large error associated with the outcome variable due to unknown and confounding factors. For example kidney disease could directly affect TWI. But, as previously mentioned, if there was kidney disease in the sample that was not known due to the lack of questions pertaining to kidney disease in the NHANES questionnaires, this variable would not be able to be controlled for in this study. This may explain why there was so much unexplained variability in the dependent variable of TWI. 
The purpose of specific aim 4 was to determine if a model, or an equation, could be derived that would better explain the variability associated with TWI. Unfortunately, the results of specific aim 4 indicated that it may not be possible to write equations which estimate water requirements using TWI as the outcome variable; only about $28 \%$ of the variability of TWI could be explained by model A and model B. Because water requirement should be affected by the same variables as energy requirements, the inability to account for a significant amount of the variance indicated that TWI may be a poor estimate of water requirements.

As discussed above, because there is no homeostatic mechanism to limit water intake, individuals may very well have ingested considerably more TWI than they required (see discussion above). Another possibility was that some individuals may have ingested less than their water requirements. It is also possible that individuals may purposefully avoid drinking despite feeling thirsty. In any case the 24-hour dietary recall was only given for one day and may not reflect the usual intake of individuals. Since individuals may be consuming substantially more or less than their water requirements, the utilization of TWI as a proxy for water requirements may have some limitations.

\section{$\underline{\text { Limitations }}$}

There are limitations inherent in using the NHANES dataset. The NHANES dataset was collected for observational cross-sectional studies. By design, the interpretation of cross-sectional studies may be limited by the inability to infer cause and effect, as the data were collected at the same time. 
For this present study the interpretation was based on correlates of TWI. Some of the correlates of TWI would have preceded the outcome variable. The variables of gender, race/ethnicity, and height would not change with time. The variables of weight, BMI, age and FFM change slowly enough to not precede TWI in time. Only the variables of osmolality and kcals ingested would be affected by a cause and effect limitation. So it was not possible to know whether an individual's osmolality or kcal intake caused an effect on TWI (if they occurred prior to TWI) or if the amount of TWI caused an osmolality or kcal ingestion effect. The cross-sectional nature of this study may be one reason why a strong prediction equation with a high $r^{2}$ could not be determined.

Another limitation of using NHANES data as opposed to a direct measure of water requirements, e.g., measuring losses, was that the analysis was limited by the available data within the dataset, which was not always the best data to answer these specific research questions. This is a problem common to the analysis of secondary data. This study was limited by the questions asked, examination performed, laboratory values collected and specific nutrients analyzed during the 24-hour dietary recall. Researchers using these datasets understand that they cannot control which data was collected. The data that would best answer the research question for a particular study may not necessarily be what was collected.

For example the NHANES did not collect some pertinent information about serious disruptions to water balance in certain segments of the population. One of the most egregious examples of this was the inability to exclude individuals with chronic kidney disease stage 5 who were receiving hemodialysis or peritoneal dialysis. These individuals would likely be anuric (have no urine output) and would likely be on a fluid 
restricted diet. This would have altered their ability to maintain water balance. NHANES did collect this type of data for the years 2001-2004 but did not collect it in 1999-2000. This was a type of inconsistency of data collection that limited the ability of the researcher to control for possible and known confounding factors. This problem was especially exaggerated by the fact that each data point was not an individual but was weighted to represent many individuals that shared similar characteristics.

The researcher who analyzes secondary data cannot control when or how data collections methods were changed. There were changes to several variables and collection protocols that occurred with every two-year cycle in order to either improve upon the data collection process or the type of data collected (CDC, 2009). For example, in some years plain drinking water was collected as part of the post-dietary recall data collection. In other two-year cycles, it was collected as part of the multiple-pass total dietary recall collection. Changes like this were sometimes explained as an improvement in data collection while other times no explanation was provided. Despite the reason for the changes, differences may exist between each two-year cycle as a result of these changes. However, in the NHANES documentation it is highly recommended to use more than one, two-year cycle (CDC, 2009).

Usual dietary intake data is best described by more than one day of data collection. There were several years in which more than one day of the dietary recall was provided. The specific six years of data in this present study (1999-2004) were selected based on the availability of all relevant variables. For example, bioelectrical impedance analysis, which was an estimate of FFM, was only collected between the years 19992004 (CDC, 2009). During these six-years of data collection, more than one day of 
dietary recall information was not collected in each of the three, 2-year cycles. This meant that for the present study only one day of data was able to be utilized for all six years. However, collecting one day of water intake data may limit the ability to determine the usual intake of water (IOM, 2005a). But, unlike other nutrients, water requirement is based on the total water lost from all routes and this replacement must be made rapidly in order to prevent dehydration (IOM, 2005a). It was therefore accepted for the purposes of this study to explain estimated water requirements using the TWI based on one day of data.

Other limitations of the methodology in this study were that other factors that could not be controlled for sufficiently, such as physical activity (PA), may have affected the amount of TWI. These other factors might have accounted for EWR not being accurate measures of actual water intake. An attempt to control for differences in PA was planned prior to the analysis using exclusion criteria. Individuals who had high or moderate PA sweated more than those who were less active and this difference could have accounted for differences in TWI between individuals (IOM, 2005a).

The only appropriate question to determine the level of PA with an acceptable amount of missing data was self-reported usual PA level (CDC, 2010a; CDC, 2010b; CDC, 2010c). Those with a self-reported highly active usual PA level were excluded from the study with the belief that they would have sweat to a greater degree and have higher insensible respiratory losses than the rest of the sample. However, there were no questions to address whether individuals who self-reported their usual level of PA as moderate or low actually had that level of PA on the day of the 24-hour dietary recall. It is possible that individuals who answered that they were usually less active were included 
in the study, but in actuality had an extremely high PA on the day of the 24-hour recall. This was not accounted for in the study because there was no information in NHANES to address this specific issue. Physical activity is also associated to other factors such as age and gender (IOM, 2005b). A self-report of active PA does not necessarily mean the same in all age groups by gender.

Another limiting factor that could not be adequately addressed but could adversely affect TWI was the environmental conditions at the location in which the individual was examined. NHANES collected data from first stage of sampling in strata representing single counties or groups of neighboring counties (CDC, 2010e). Strata were formed based on geography and the proportions of minority populations with the probability of selection proportional to a measure of size (CDC, 2010e). Strata were located throughout the country (CDC, 2010e).

It is possible that several strata came from southern counties in the summertime while others came from northern counties in the wintertime. If this were the case, there may have been differences between individuals in terms of sweating rates based on their environmental conditions. This information was not known and could therefore not be accounted for in the design of the study. The assumption in this study was that despite possible differences in environmental conditions the individuals in this study were in a comfortable setting in which little sweating occurred. If this was not the case, a systematic error of higher water intake from those in southern counties may have occurred and confounded the results. The only control over this situation was to combine as many two-year cycles as possible to obtain a large sample size and to assume that there was a random selection of individuals throughout the country and between seasons. 


\section{$\underline{\text { Recommendations for future research }}$}

Recommendations for future research are to directly measure the water requirement in a sample of people and then determine if any of the existing formulas or a newly derived formula can adequately predict the individual's water requirements. A water balance study or an isotope dilution study would be the gold standard in the determination of water requirements. In a water balance study, the water requirement is the amount of water needed to replace losses from all routes (i.e. urine, GI, sweat, and insensible transcutaneous and respiratory losses) minus free water reserve in the urine (IOM, 2005a; Manz et al., 2002). The free water reserve is the water in urine that is in excess of the amount of urine required to excrete the renal solute load (Manz et al., 2002). In an isotope dilution study the water requirement is the amount of water needed based on the water turnover rate (IOM, 2005a; Raman et al., 2004). The water turnover rate is determined using labeled water that is diluted by other sources of water intake over time.

Once a water balance or isotope dilution study provided an accurate determination of water requirements, the current equations could be evaluated as to their validity and reliability in predicting this requirement. If one of these equations agreed with either of these outcome variables, it could be the practical equation of choice for determining water requirements across healthcare settings. Otherwise, water balance or isotope dilution studies could be the estimate of water requirements against which a new valid prediction equation could be written. These types of studies could be extended to groups of individuals who may have differing water requirements, such as older adults, obese individuals and those in different ethnic groups. 


\section{CHAPTER VI.}

\section{CONCLUSION}

The aims of this study were first to determine if there was an agreement among EWR equations and then between EWR equations and TWI. In this study TWI was considered as an estimate of water requirements.

This study revealed that none of the five equations being utilized in clinical practice to estimate water requirements agreed with each other. This finding provides little assurance that practitioners calculate a good estimate of an individual's water requirement on which to base their interventions. Further, it is reasonable to assume that estimates of water requirements will vary based on the specific equation selected within a care setting and on the specific equation selected by RDs between care settings, resulting in a discrepancy in the amount of water recommended by RDs in their nutrition intervention.

The results of specific aim 2 revealed that TWI did not agree with EWR equations currently used in clinical practice. Total water intake was selected as the estimate of water requirements based on the physiological mechanism of thirst as stated by the IOM. It is thirst which drives sufficient water intake in order to meet minimum water requirements. There is no opposing physiological mechanism to halt the consumption of water in amounts far greater than required. Total water intake in amounts substantially greater or less than minimum requirements would affect TWI. Since TWI did not agree with EWR equations, either individuals may not have consumed a volume of water close to their water requirements in this study or an improved equation was needed to predict TWI. 
FFM was found to have a weak and positive association to TWI. That means that individuals with more FFM had more TWI. This was especially true in underweight individuals. This is likely due to the relatively larger fat-free to fat ratio in the underweight and because FFM is more highly hydrated than fat mass. These results provided clear justification to include FFM in models developed to predict TWI.

The last aim consisted of the creation of two models to predict TWI. Model A included FFM while model B did not. Both final models contained six variables. The commonalities of the models were in the variables of race/ethnicity, kcals, age, and height. Model A (with FFM) also included gender. Model B (without FFM) also included BMI and osmolality. However, neither model explained more than $28 \%$ of the variability in TWI, indicating that it may not be possible to predict the TWI of individuals with the practical variables considered in this study.

The lack of agreement between any of the existing equations and TWI in Aim 2 and an inability to account for more than $28 \%$ of the variance in Aim 4 may be a consequence of the limitations of the methods employed in this study. The IOM stated that TWI could be an estimate of water requirements, but TWI may not be a good estimate of water requirements in this study because individuals may have consumed substantially more or less than the minimal amount of water as dictated by the physiological drive for thirst. In order to determine if any of the EWR equations predict water requirements, a better and more valid technique to estimate water requirements is needed.

Future studies should utilize a gold standard technique to measure water requirements. The approaches that are considered as the gold standard for estimating 
water requirements are water balance studies or isotope dilution studies. This would allow the creation of more accurate evidence-based equations to predict the water requirements of individuals, which could become the standard in clinical practice. 


\section{LIST OF REFERENCES}

Abdallah, L. Remington, R., Houde, S., Zhan, L, \& Melillo K.D. (2009). Dehydration reduction in community-dwelling older adults. Research in Gerontological Nursing, 2, 49-57.

Adolph, E.F. (1933). The metabolism and distribution of water in body and tissues. Physiological Reviews, 13, 336-363.

Akner, G., \& Floistrup, H. (2003). Individual assessment of intake of energy, nutrients and water in 54 elderly multi-diseased nursing-home residents. Journal of Nutrition Health and Aging, 7, 2-12.

Amato, P. Keating, K. P., Quercia, R. A., \& Karbonic, J. (1995). Equationic methods of estimating calorie requirements in mechanically ventilated obese patients: A reappraisal. Nutrition in Clinical Practice, 10, 229-232.

American Dietetic Association. (2007). What is the best clinical and/or biochemical parameter for hydration status in the adult (19-64 years)? Retrieved August 3, 2010, from http://www.adaevidencelibrary.com/conclusion.cfm?conclusion_statement_id=25 0805

Armstrong, L.E. (2005). Hydration assessment techniques. Nutrition Reviews, 11, S40S54.

Armstrong, L.E. (2007). Assessing hydration status: The elusive gold standard. Journal of the American College of Nutrition, 26(5), 575S-584S.

Armstrong, L.E., Maresh, C.M., Castellani, J.W., Bergeron, M.F., Kenefick, R.W., LaGasse, K.E., \& Riebe, D. (1994). Urinary indices of hydration status. International Journal of Sports Nutrition, 4, 265-279.

Armstrong, L.E., Whittlesey, M.J., Casa, D.J., Elliott, T.A., Kavouras, S.A., Keith, N.R., \& Maresh, C.M. (2006). No effect of 5\% hypohydration on running economy of competitive runners at 23degrees Celsius. Medicine and Science in Sports and Exercise, 38(10), 1762-1769.

Armstrong-Esther, C.A., Browne, K.D., Armstrong-Esther, D.C., \& Sander, L. (1996). The institutionalized elderly: Dry to the bone! International Journal of Nursing Studies, 33(6), 619-628.

Barrett, K.E., \& Raybould, H.E. (2008). Gastrointestinal physiology. In B. Koeppen and B. Stanton (Eds.), Berne \& Levy physiology sixth ed. (pp. 485-542). Philadelphia, PA: Mosby Elsevier, Inc. 
Bassett, S.H., \& Van Alstine, H.E. (1934). Mineral exchanges of man: Variations in the mineral content of diets with a constant raw weight equation. Journal of Nutrition, $9(2), 175-189$.

Baumgartner, R.N. (2000). Body composition in healthy aging. Annals of the New York Academy of Sciences, 904, 437-448.

Bayliss, P.H. \& Thompson, C.J. (1988). Osmoregulation of vasopressin in secretion and thirst in health and disease. Clinical Endocrinology, 29, 549-576.

Beisalski, H.K., Bischoff, S.C., Boehles, H.J., \& Muehlhoefer, A. (2009). Water, electrolytes, vitamins and trace elements- guidelines on parenteral nutrition, Chapter 7. German Medical Science, 7, 1-7.

Benjamini, Y. and Hochberg, Y. (1995). Controlling the false discovery rate: a practical and powerful approach to multiple testing. Journal of the Royal Statistical Society, Series B, 57, 289-300.

Berl, T. (2008). Impact of solute intake on urine flow and water excretion. Journal of the American Society of Nephrology, 19, 1076-1078.

Bland, J.M., \& Altman, D.G. (1986). Statistical methods for assessing agreement between two methods of clinical measurement. Lancet, i, 307-310.

Boschmann, M., Steiniger, J., Hille, U., Tank, J., Adams, F., Sharma, A.M., Klaus, S., Luft, F.C., \& Jordan, J. (2003). Water-induced thermogenesis. Journal of Clinical Endocrinology \& Metabolism, 88, 6015-6019.

Bossingham, M.J., Carnell, N.S., \& Campbell, W.W. (2005). Water balance, hydration status, and fat-free mass hydration in younger and older adults. American Journal of Clinical Nutrition, 81, 1342-1350.

Campbell, S.M. (2007). Hydration needs throughout the lifespan. Journal of the American College of Nutrition, 26(5) 585S-587S.

Candela, L., \& Yucha, C. (2004). Renal regulation of extracellular fluid volume and osmolality. Nephrology Nursing Journal, 31, 397-444.

Castellanos, V.H., Silver, H.J., Gallagher-Allred, C., \& Smith, T.R. (2003). Nutrition issues in the home, community, and long-term care. Nutrition in Clinical Practice, 18, 21-36.

Centers for Disease Control and Prevention (CDC) (2010a). National Center for Health Statistics (NCHS) National Health and Nutrition Examination Survey Data Hyattsville, MD: U.S. Department of Health and Human Services, Centers for 
Disease Control and Prevention 1999-2000. Retrieved August 3, 2010, from http://www.cdc.gov/nchs/nhanes/nhanes1999-2000/nhanes99_00.htm

Centers for Disease Control and Prevention (CDC) (2010b). National Center for Health Statistics (NCHS) National Health and Nutrition Examination Survey Data Hyattsville, MD: U.S. Department of Health and Human Services, Centers for Disease Control and Prevention 2001-2002. Retrieved August 3, 2010, from http://www.cdc.gov/nchs/nhanes/nhanes2001-2002/nhanes01_02.htm

Centers for Disease Control and Prevention (CDC) (2010c). National Center for Health Statistics (NCHS) National Health and Nutrition Examination Survey Data Hyattsville, MD: U.S. Department of Health and Human Services, Centers for Disease Control and Prevention 2003-2004. Retrieved August 3, 2010, from http://www.cdc.gov/nchs/nhanes/nhanes2003-2004/nhanes03_04.htm

Centers for Disease Control and Prevention (CDC) (2010d). National Center for Health Statistics (NCHS) National Health and Nutrition Examination Survey Data Hyattsville, MD: U.S. Department of Health and Human Services, Centers for Disease Control and Prevention 2005-2006. Retrieved August 3, 2010, from http://www.cdc.gov/nchs/nhanes/nhanes2005-2006/nhanes05_06.htm

Centers for Disease Control and Prevention (CDC) (2010e). National Center for Health Statistics (NCHS) National Health and Nutrition Examination Survey Data Hyattsville, MD: U.S. Department of Health and Human Services, Centers for Disease Control and Prevention Retrieved August 3, 2010, from http://www.cdc.gov/nchs/tutorials/Nhanes/SurveyDesign/SampleDesign/Info1.ht $\mathrm{m}$

Centers for Disease Control and Prevention (CDC) (2010f). National Center for Health Statistics (NCHS) National Health and Nutrition Examination Survey Data Hyattsville, MD: U.S. Department of Health and Human Services, Centers for Disease Control and Prevention Retrieved August 3, 2010, from http://www.cdc.gov/nchs/nhanes/about_nhanes.htm

Centers for Disease Control and Prevention (CDC) (2009). National Center for Health Statistics (NCHS) National Health and Nutrition Examination Survey Analytic and Reporting Guidelines 1999-2004. Hyattsville, MD: U.S. Department of Health and Human Services, Centers for Disease Control and Prevention Retrieved August 3, 2010, from http://www.cdc.gov/nchs/nhanes/nhanes_questionnaires.htm

Centers for Disease Control and Prevention (CDC) (2006). National Center for Health Statistics (NCHS) National Health and Nutrition Examination Survey Questionnaires 1999-2004. Hyattsville, MD: U.S. Department of Health and Human Services, Centers for Disease Control and Prevention Retrieved August 3, 
2010, from

http://www.cdc.gov/nchs/data/nhanes/nhanes_03_04/nhanes_analytic_guidelines_ dec_2005.pdf

Centers for Disease Control and Prevention (CDC). (2009). Prevalence of diabetes by age, 1980-2007. Retrieved August 3, 2010, from http://www.cdc.gov/Diabetes/statistics/prev/national/figbyage.htm

Chernoff, R. (1994a). Nutritional requirements and physiological changes in aging. Nutrition Reviews, 52(8), S3-S5.

Chernoff, R. (1994b). Meeting the nutritional needs of the elderly institutionalized setting. Nutrition Reviews, 52(4), 132-136.

Chidester, J.C., \& Spangler, A.A. (1997). Fluid intake in the institutionalized elderly. Journal of the American Dietetic Association, 97, 23-28.

Claros, G., Hull, H.R., \& Fields, D.A. (2005). Comparison of air displacement plethysmography to hydrostatic weighing for estimating total body density in children. BioMed Central Pediatrics, 5, 1-9.

Cody, R.P., \& Smith, J.K. (2006). Multiple regression analysis. In R.P. Cody and J.K. Smith (Eds.). Applied statistics and the SAS programming language fifth ed. (pp. 282-315). Upper Saddle River, NJ: Pearson Education, Inc.

Cole, K.S. (1997). Membranes, ions, and impulses. Berkley, CA: University of California Press.

Consolazio, C.F., Matoush, L.O., Johnson, H.L., \& Daws, T.A. (1968). Protein and water balance of young adults during prolonged exposure to high altitude (4300 meters). American Journal of Clinical Nutrition, 21, 154-161.

Crecelius, C. (2008). Dehydration: Myth and reality. Journal of the American Medical Directors Association, 9, 287-288.

Da Rocha, E.E.M., Alves, V.G.F., Silva, M.H.N., Chiesa, C.A., \& da Fonesca, R.B.V. (2005). Can measured resting energy expenditure be estimated by equation in daily nutrition practice? Current Opinion in Clinical Nutrition Metabolic Care, 8(3), 319-328.

Darrow, D.C., and Pratt, E.L. (1950). Fluid therapy; relation to tissue composition and expenditure of water and electrolyte; Council on Food and Nutrition. Journal of the American Medical Association, 143, 432-439. 
De Castro, J.M. (1988). A microregulatory analysis of spontaneous fluid intake by humans: Evidence that the amount of liquid ingested and its timing is mainly governed by feeding. Physiology and Behavior, 43, 705-714.

De Castro, J.M. (1992). Age-related changes in natural spontaneous fluid ingestion and thirst in humans. Journal of Gerontology, 47(5), P321-P330.

Dickerson, R.N., \& Brown, R.O. (2005). Long-term enteral nutrition support and the risk of dehydration. Nutrition in Clinical Practice, 20, 646-653.

Dimant, J. (2001). Delivery of nutrition and hydration care in nursing homes: Assessment and interventions to prevent and treat dehydration, malnutrition, and weight loss. Journal of the American Medical Directors Association, 2(4), 175-182.

Doria, M.F. (2006). Bottled water vs. tap water: Understanding consumers' preferences. Journal of Water and Health, 4, 271-276.

Federal Interagency Forum on Aging-Related Statistics. Older Americans 2008: Key Indicators of Well-Being (March, 2008). Federal Interagency Forum on AgingRelated Statistics. Washington, DC: U.S. Government Printing Office.

Feinsod, F.M., Levenson, S.A., Rapp, K., Rapp, M.P., Beechinor, E., \& Liebmann, L. (2004). Dehydration in frail, older residents in long-term care facilities. Journal of the American Medical Directors Association, 3(6), 371-376.

Ferry, M. (2005). Strategies for ensuring good hydration in the elderly. Nutrition Reviews, 63, S22-S29.

Fletcher, S.J., Slaymaker, A.E., Bodenham A.R., \& Vucevic, M. (1999). Urine colour as an index of hydration in critically ill patients. Anaesthesia, 54, 172-197.

Food and Nutrition Board National Research Council. (1989). Recommended dietary allowances: $10^{\text {th }}$ Edition. Washington, DC: National Academy Press.

Frankenfield, D., Roth-Yousey, L., \& Compher, C. (2005). Comparison of predictive equations for resting metabolic rate in healthy non-obese and obese adults: A systematic review. Journal of the American Dietetic Association, 105, 775-789.

Frankenfield, D., Rowe, W.A., Smith, J.S., \& Cooney, R.N. (2003). Validation of several established equations for resting metabolic rate in obese and non-obese people. Journal of the American Dietetic Association, 103, 1152-1159.

Fulgoni, V.L. (2007). Limitations of data on fluid intake. Journal of the American College of Nutrition, 26(5), 588S-591S. 
Fuller, N.J., Sawyer, M.B., \& Elia, M. (1994). Comparative evaluation of body composition methods and predictions, and calculation of density and hydration fraction of fat-free mass, in obese women. Journal of Obesity and Related Metabolic Disorders, 18, 503-512.

Gaillard, C., Alix, E., Salle, A., Berrut, G., \& Ritz, P. (2007). Energy requirements in frail elderly people: A review of the literature. Clinical Nutrition, 26, 16-24.

Gans, K.M., Risica, P.M., Kirtania, U., Jennings, A., Strolla, L.O., Steiner-Asiedu, M., Hardy, N., and Lasater, T.M. (2009). Dietary Behaviors and Portion Sizes of Black Women Who Enrolled in SisterTalk and Variation by Demographic Characteristics. Journal of Nutrition Education and Behavior, 41, 32-40.

Goran, M.I., Driscoll, P., Johnson, R., Nagy, T.R., \& Hunter, G. (1996). Cross-calibration of body-composition techniques against dual-energy X-ray absorptiometry in young children. American Journal of Clinical Nutrition, 63, 299-305.

Grandjean, A.C., \& Campbell, S.M. (2004). Hydration: Fluids for life. A monograph by the North American Branch of the International Life Science Institute. Washington DC: ILSA North America.

Grandjean, A.C., Reimers, K.J., Bannick, M.A., \& Haven, M.C. (2000). The effect of caffeinated, non-caffeinated, caloric, and non-caloric beverages on hydration. Journal of the American College of Nutrition, 19(5), 591-600.

Grandjean, A.C., Reimers, K.J., \& Buyckx, M.E. (2003). Hydration: Issues for the $21^{\text {st }}$ century. Nutrition Reviews, 61(8), 261-271.

Grant, A., \& DeHoog, S. (1999). In A. Grant and S. DeHoog (Eds.) Nutrition Assessment support and management fifth edition. (pp.287-288). Seattle, WA: Grant and DeHoog.

Greenleaf, J.E., Bernauer, E.M., Juhos, L.T., Young, H.L., Morse, J.T., \& Staley, R.W. (1977). Effects of exercise on fluid exchange and body composition in man during 14-day bed rest. Journal of Applied Physiology, 43, 126-132.

Gropper, S.S., Smith, J.L., \& Groff, J.L. (2009). In S.S. Gropper, J.L. Smith, and J.L. Groff (Eds.) Advanced Nutrition and Human Metabolism fifth edition. (pp. 279307). Belmont, CA: Wadsworth Cengage Learning.

Gross, C.R., Lindquist, R.D., Woolley, A.C., Granieri, R., Allard, K., \& Webster, B. (1992). Clinical indicators of dehydration severity in elderly patients. Journal of Emergency Medicine, 10, 267-274. 
Gudivaka, R., Schoeller, D.A., Kushner, R.F., \& Bolt, M.J.G. (1999). Single- and multifrequency models for bioelectrical impedance analysis. Journal of Applied Physiology, 87, 1087-1096.

Guyton, A.C., \& Hall, J.E. (2006). The body fluids and kidneys. In A. Guyton and J. Hall (Eds.). Textbook of medical physiology eleventh ed. (pp. 291-415). Philadelphia, PA: Saunders Elsevier, Inc.

Haarbo, J., Gotfredsen, A., Hassager, C., \&Christiansen, C. (1991). Validation of body composition by dual-energy X-ray absorptiometry (DEXA). Clinical Physiology, $11,331-341$.

Hammond, K.A. (2004). Dietary and clinical assessment. In L.K. Mahan \& S.E. Stump (Eds.), Krause's food nutrition and diet therapy eleventh ed. (pp. 407-435). Philadelphia, PA: Saunders.

Han, S.S., Kim, K.W., Kim, K.I., Na, K.Y., Chae, D.W., Kim, S., \& Chin, H.J. (2010). Lean mass index: A better predictor of mortality than body mass index in elderly Asians. Journal of the American Geriatrics Society, 58, 312-317.

Harris, J.A., \& Benedict, F.G. (1919). A biometric study of basal metabolism in man. Publication no. 279. Washington, DC: Carnegie Institute of Washington.

Ho, S.N. (2006). Intracellular water homeostasis and the mammalian cellular osmotic stress response. Journal of Cellular Physiology, 206, 9-15.

Hodak, S.P., \& Verbalis, J.G. (2005). Abnormalities of water homeostasis in aging. Endocrinology and Metabolism Clinics, 34, 1-12.

Holben, D.H., Hassell, J.T., Williams, J.L., \& Helle, B. (1999). Fluid intake compared with established standards and symptoms of dehydration among elderly residents of a long-term-care facility. Journal of the American Dietetic Association, 99(11), 1447-1450.

Holliday, M.A., \& Segar, W.E. (1957). The maintenance need for water in parenteral fluid therapy. Pediatrics, 19, 823-832.

Houston, D.K., Nicklas, B.J., \& Zizza, A. (2009). Weighty concerns: The growing prevalence of obesity among older adults. Journal of the American Dietetic Association, 109, 1886-1895.

Huang, T.T., Johnson, M.S., \& Goran, M.I. (2002). Development of a prediction equation for insulin sensitivity from anthropometry and fasting insulin in prepubertal and early pubertal children. Diabetes Care, 25, 1203-1210. 
Huang, T.T., Watkins, M.P., \& Goran, M.I. (2003). Predicting total body fat from anthropometry in Latino children. Obesity Research, 11(10), 1192-1199.

International Dietetics and Nutrition Terminology Reference Manual: Standardized Language for the Nutrition Care Process, First Edition. (2008). Chicago, IL: American Dietetic Association.

International Dietetics and Nutrition Terminology Reference Manual: Standardized Language for the Nutrition Care Process, Second edition. (2009). Chicago, IL: American Dietetic Association.

Institute of Medicine, Food and Nutrition Board. (2005a). Dietary Reference Intakes for water, potassium, sodium, chloride \& sulfate, Washington, DC: The National Academies Press.

Institute of Medicine, Food and Nutrition Board. (2005b). Dietary Reference Intakes for energy, carbohydrate, fiber, fat, fatty acids, cholesterol, protein, and amino acids. Washington, DC: The National Academies Press.

Janssen, I., Heymsfield, S.B., Baumgartner, R.N., \& Ross, R. (2000). Estimation of skeletal muscle mass by bioelectrical impedance analysis. Journal of Applied Physiology, 89, 465-471.

Jequier, E., \& Constant, F. (2010). Water as an essential nutrient: The physiological basis of hydration. European Journal of Clinical Nutrition, 64, 115-123.

Johnson, A.G., Crawford, G.A., Kelly, D., Nguyen, T.V., \& Gyory, A.Z. (1994).

Arginine vasopressin and osmolality in the elderly. Journal of the American Geriatric

Society, 42, 399-404.

Juan W.Y., \& Bastiotos, P.P. (2002). USDA Center for Nutrition Policy and Promotion. More than one in three older Americans many not drink enough water: Nutrition Insights. Retrieved August 3, 2010 from

http://www.cnpp.usda.gov/Publications/NutritionInsights/Insight27.pdf

Kanellakis, S., Kourlaba, G., Moschonis, G., Vandorou, A., \& Manios, Y. (2010). Development and validation of two equations estimating body composition for overweight and obese postmenopausal women. Maturitas, 65, 64-68.

Kant, A.K., Graubard, B.I., \& Atchison, E.A. (2009). Intakes of plain water, moisture in foods and beverages, and total water in the adult US population-nutritional, meal pattern, and body weight correlates: National Health and Nutrition Examination Surveys 1999-2006. American Journal of Clinical Nutrition, 90, 655-663. 
Kavouras, S.A. (2002). Assessing hydration status. Current Opinion in Clinical Nutrition and Metabolic Care, 5, 519-524.

Kayser-Jones, J., Schell, E.S., Porter, C., Barbaccia, J.C., \& Shaw, H. (1999). Factors contributing to dehydration in nursing homes: Inadequate staffing and lack of professional supervision. Journal of the American Geriatrics Society, 47, 11871194.

Kenney, W.L., \& Chiu, P. (2001). Influence of age on thirst and fluid intake. Medicine and Science in Sports and Exercise, 33(9), 1524-1532.

Kleinbaum, D.G., Kupper, L. L., Nizam, A., \& Muller, K. E. (2008). Applied regression analysis and other multivariable methods, Fourth Ed. Pacific Grove, CA: Duxbury Press.

Kleiner, S.M. (1999). Water: an essential but overlooked nutrient. Journal of the American Dietetic Association, 99, 200-206.

Koea, J.B., Wolfe, R.R., \& Shaw, J.H. (1995). Total energy expenditure during total parenteral nutrition: Ambulatory patients at home versus patients with sepsis in surgical intensive care. Surgery, 118(1), 54-62.

Koeppen, B.M., \& Stanton, B.A. (2008). Homeostasis of body fluids. In B. Koeppen, B. Stanton (Eds.), Berne and Levy physiology sixth ed. (pp. 20-33).Philadelphia, PA: Mosby Elsevier.

Kolasa, K.M., Lackey, C.J., \& Grandjean, A.C. (2009). Hydration and health promotion. Nutrition Today, 44(5), 190-201.

Krenitsky, J. (2005). Adjusted body weight, pro: Evidence to support the use of adjusted body weight in calculating calorie requirements. Nutrition in Clinical Practice, 20, 468-473.

Kyle, U.G., Bosaeus, I., De Lorenzo, A.D., Deurenberg, P., Elia, M., Gomez, J.M., Heitmann, L. B., Kent-Smith, L., Melchior, J.C., Pirlich, M., Scharfetter, H., Schols, A.M.W.J., \& Pichard, C. (2004). Bioelectrical impedance analysis - part II: Utilization in clinical practice. Clinical Nutrition, 23, 1430-1453.

Kyle, U.G., Genton, L., Hans, D., Karsegard, V.L., Michel, J.P., Slosman, D.O., \& Pichard, C. (2001). Total body mass, fat mass, fat-free mass and skeletal muscle in older people: Cross-sectional differences in 60 year-old persons. Journal of the American Geriatric Society, 49, 1633-1640. 
Lacey, K., \& Pritchett, E. (2003). Nutrition care process and model: ADA adopts road map to quality care and outcomes management. Journal of the American Dietetic Association, 103, 1061-1072.

Lane, H.W., Gretebeck, R.J., Schoeller, D.A., Davis-Street, J., Socki, R.A., \& Gibson, E.K. (1997). Comparison of ground-based and space flight energy expenditure and water turnover in middle-aged healthy male US astronauts. American Journal of Clinical Nutrition, 65, 4-12.

Lappalainen, R., Mennen, L., van Weert, L., \& Mykkanen, H. (1993). Drinking water with a meal: A simple method of coping with feelings of hunger, satiety and desire to eat. European Journal of Clinical Nutrition, 47, 815-819.

Lapane, K.L., \& Resnik, L. (2005). Obesity in nursing homes: An escalating problem. Journal of the American Geriatrics Society, 53, 1386-1391.

Lee, S.Y., \& Gallagher, D. (2008). Assessment methods in human body composition. Current Opinion in Clinical Nutrition and Metabolic Care, 11, 566-572.

Lichtenstein, A.H., Rasmussen, H., Yu, W.W., Epstein, S.R., \& Russell, R.M. (2008). Modified MyPyramid for older adults. Journal of Nutrition, 138, 5-11.

Lipp, J., Lord, L.M., \& Scholer, L.H. (1999). Fluid management in enteral nutrition. Nutrition in Clinical Practice, 14, 232-237.

Louden, J.D. (2009). Regulation of fluid and electrolyte balance. Anaesthesia and Intensive Care, 10(6), 279-285.

Luckey, A.E., \& Parsa, C.J. (2003). Fluid and electrolytes in the aged. Archives of Surgery, 138, 1055-1060.

Madea, B., \& Lachenmeirer, D.W. (2005). Postmortem diagnosis of hypertonic dehydration. Forensic Science International, 155, 1-6.

Mager, J.R., Sibley, S.D., Beckman, T.R., Kellogg, T.A., \& Earthman, C.P. (2008). Multifrequency bioelectrical impedance analysis and bioimpedance spectroscopy for monitoring fluid and body cell mass changes after gastric bypass surgery. Clinical Nutrition, 27, 832-841.

Mange, K., Matsuura, D., Cizman, B., Soto, H., Ziyadeh, F.N., Goldfarb, S., \& Neilson, E.G. (1997). Language guiding therapy; the case of dehydration versus volume depletion. Annals of Internal Medicine, 127, 848-853.

Manz, F., \& Wentz, A. (2003). 24-h hydration status: parameters, epidemiology and recommendations. European Journal of Clinical Nutrition, 57(2), S10-S18. 
Manz, F., \& Wentz, A. (2005). Hydration status in the United States and Germany. Nutrition Reviews, 63(6), S55-S62.

Manz, F., Wentz, A., \& Sichert-Hellert, W. (2002). The most essential nutrient: Defining the adequate intake of water. Journal of Pediatrics, 141, 587-592.

Martin, A.D., Daniel, M.Z., Drinkwater, D.T., \& Clarys, J.P. (1994). Adipose tissue density, estimated adipose lipid fraction and whole body adiposity in male cadavers. International Journal of Obesity, 18(2), 79-83.

Martin, W.H., Cerundolo, L.H., Pikosky, M.A., Gaine, P.C., Maresh, C.M., Armstrong, L.E., Bolster, D.R., \& Rodriguez, N.R. (2006). Effects of dietary protein intake on indexes of hydration. Journal of the American Dietetic Association, 106, 587-589.

Mathieu, J., Foust, M., \& Ouelltet, P. (2005). Implementing nutrition diagnosis, step two in the nutrition care process and model: Challenges and lessons learned in two health care facilities. Journal of the American Dietetic Association, 105, 16361640 .

McGee, S., Abernethy, W.B., \& Simel, D.L. (1999). Is this patient hypovolemic? Journal of the American Medical Association, 281, 1022-1029.

McKnight, P.R., McKnight, K., Souraya, S., Figueredo, A.J. (2007). Missing Data: A Gentle Introduction. New York, NY: The Guilford Press.

Mentes, J.C., Wakefield, B., \& Culp, K. (2006). Use of a urine color chart to monitor hydration status in nursing home residents. Biological Research for Nursing, 7(3), 197-203.

Mifflin, M.D., St Jeor, S.T., Hill, L.A., Scott, B.J., Daugherty, S.A., \& Koh, Y.O. (1990). A new predictive equation for resting energy expenditure in healthy individuals. American Journal of Clinical Nutrition, 51, 241-247.

Mingrone, G., Bertuzzi, A., Capristo, E., Greco, A.V., Manco, M., Pietrobelli, A., Salinari, S., \& Heymsfield, S.B. (2001). Unreliable use of standard muscle hydration value in obesity. American Journal of Physiology. Endocrinology and Metabolism, 280, E365-E371.

Montain, S.J., Laird, J.E., Latzka, W.A., \& Sawka, M.N. (1997). Aldosterone and vasopressin responses in the heat: hydration level and exercise intensity effect. Medicine and Science in Sports and Exercise, 29(5), 661-668.

Neiheisel, M.B., Sudduth, A.L., \& Schmidt Luggen, A. (2010). Endocrine and metabolic alterations and nutrition in the older adult. In M. Bernstein and A. Schmidt 
Luggen (Eds.). Nutrition for the older adult (pp. 181-208). Sudbury, MA: Jones and Bartlett.

Nielsen, S.J., \& Popkin, B.M. (2004). Changes in beverage intake between 1977 and 2001. American Journal of Preventative Medicine, 27(3), 205-210.

Nieuwenhuizen, W.F., Weenen, H., Rigby, P., \& Hetherington, M.M. (2010). Older adults and patients in need of nutritional support: Review of current treatment options and factors influencing nutritional intake. Clinical Nutrition, 29, 160-169.

Noakes, T.D., Wilson, G., Gray, D.A., Lambert, M.I., \& Dennis, S.C. (2001). Peak rates of diuresis in healthy humans during oral fluid overload. South African Medical Journal, 91, 852-857.

Ogden, C.L., Carroll, M.D., McDowell, M.A., \& Flegal, K.M. (2007). Obesity among adults in the United States- no change since 2003-2004. NCHS data brief no.1. Hyattsville, MD, National Center for Health Statistics.

Overholser, B.R., \& Sowinski, K.M. (2008). Biostatistics Primer: Part 2. Nutrition in Clinical Practice, 23, 76-84.

Pappano, A.J. (2008). Properties of the vasculature. In B. Koeppen \& B. Stanton (Eds.), Berne and Levy physiology sixth ed. (pp.330-369). Philadelphia, PA: Mosby Elsevier.

Patel, R.V., Matthie, J.R., Withers, P.O., Peterson, E.L., \& Zarowitz, B.J. (1994). Estimation of total body and extracellular water using single- and multiplefrequency bioimpedance. The Annals of Pharmacotherapy, 28, 565-569.

Paterna, S., Parrinello G., Cannizzaro, S., Fasullo, S., Torres, D., Sarullo, F.M., \& Di Pasquale, P. (2009). Medium term effects of different dosage of diuretic, sodium, and fluid administration on neurohormonal and clinical outcome in patients with recently compensated heart failure. American Journal of Cardiology, 103, 93-102.

Paterna, S., Parrinello, G., Fasullo, S., Sarullo, F.M., \& Di Pasquale, P. (2008). Normal sodium diet versus low sodium diet in compensated congestive heart failure: Is sodium an old enemy or a new friend? Clinical Science, 114, 221-230.

Peters, J.C. (2006). Control of energy balance. In M. Stipanuk (Ed.), Biochemical, physiological, molecular aspects of human nutrition (pp. 618-639.). St. Louis, Missouri: Saunders Elsevier, Inc.

Phillips, P.A., Bretherton, M., \& Johnston, C.I. (1991). Reduced osmotic thirst in healthy elderly men. American Journal of Physiology, 261, R166-R171. 
Phillips, P.A., Phii, D., Rolls, B.J., Ledingham, J.G., Forsling, M.L., Morton, J.J., Crowe, M.J., \& Wollner, L. (1984). Reduced thirst after water deprivation in healthy elderly men. New England Journal of Medicine, 311, 753-759.

Pietrobelli, A., \& Heysmfield, S.B. (2002). Establishing body composition in obesity. Journal of Endocrinological Investigation, 25, 884-892.

Plank, L.D., Monk, D.N., Woollard, G.A., \& Hill, G.L. (1998). Evaluation of multifrequency bioimpedance spectroscopy for measurement of the extracellular water space in critically ill patients. Applied Radiation and Isotopes, 49, 481-483.

Popkin, B.M. (2010). Pattern of beverage use across the lifecycle. Physiology and Behavior, 100, 4-9.

Popkin, B.M., Barclay, D.V., \& Nielsen, S.J. (2005). Water and food consumption patterns of US adults from 1999 to 2001. Obesity Research, 13(12), 2146-2152.

Popowski, L. A., Oplliger, R. A., Lambert G.P., Johnson, R.F., Johnson, A.K., \& Gisolfi, C.V. (2001). Blood and urinary measures of hydration status during progressive acute dehydration. Medicine and Science in Sports and Exercise, 33(5), 747-753.

Raman, A., Schoeller, D.A., Subar, A.F., Troiano, R.P., Schatzkin, A. Harris, T., Bauer, D., Bingham, S.A., Everhart, J.E., Newman, A.B., \& Tylavsky, F.A. (2004). Water turnover in 458 American adults 40-79 year of age. American Journal of Physiology. Renal Physiology, 286, F394-F401.

Ritz, P. (2000). Body water spaces and cellular hydration during healthy aging. Annals of the New York Academy of Sciences, 904, 474-483.

Ritz, P., \& Berrut, G. (2005). The importance of food hydration for day-to-day health. Nutrition Reviews, 63(6), S6-S13.

Ritz, P., Vol, S., Berrut, G., Tack, I., Arnaud, M.J., \& Tichet, J. (2008). Influence of gender and body composition on hydration and water spaces. Clinical Nutrition, $27,740-746$.

Rivlin, R.S. (2007). Keeping the young-elderly healthy: is it too late to improve our health through nutrition? American Journal of Clinical Nutrition, 86, 1572S1576 S.

Robinson, B.E., \& Weber, H. (2004). Dehydration despite drinking: beyond the BUN/creatinine ratio. Journal of the American Medical Directors Association, 5, S67-S71. 
Rolls, B.J., Bell, E.A., \& Thorwart, M.L. (1999). Water incorporated into a food but not served with a food decreases energy intake in lean women. American Journal of Clinical Nutrition, 70, 448-455.

Sansevero, A.C. (1997). Dehydration in the elderly: Strategies for prevention and management. The Nurse Practitioner, 22(4), 41-66.

Sarhill, N., Mahmoud, F.A., Christie, R., \& Tahir, A. (2003). Assessment of nutritional status and fluid deficits in advanced cancer. The American Journal of Hospice and Palliative Care, 20, 465-473.

Sartorio, A., Malavolti, M., Agosti, F., Marinone, P.G., Caiti, O., Battistini, N., \& Bedogni, G. (2005). Body water distribution in severe obesity and its assessment from eight-polar bioelectrical impedance analysis. European Journal of Clinical Nutrition, 59, 155-160.

Sawka, M.N., Cheuvront, S.N., \& Carter R. (2005). Human water needs. Nutrition Reviews, 63(6), S30-S39.

Scalera, G. (2000). Taste preference and acceptance in thirsty and dehydrated rats. Physiology and Behavior, 71, 457-468.

Schlanger, L.E., Bailey, J.L., \& Sands, J.M. (2010). Electrolytes in the aging. Advances in Chronic Kidney Disease, 17(4), 308-319.

Schoeller, D.A. (1989). Changes in total body water with age. American Journal of Clinical Nutrition, 50, 1176S-1181S.

Schols, J.M., DeGroot, C.P., Van Der Cammen, T.J., \& Olde Rikkert, M.G. (2009). Preventing and treating dehydration in the elderly during periods of illness and warm weather. Journal of Nutrition, Health and Aging, 13(2), 150-157.

Schrier, R.W. (2006). Body water homeostasis: Clinical disorders of urinary dilution and concentration. Journal of the American Society of Nephrology, 17, 1820-1832.

Schut, A., Dascenco, V., Giraud, K., Chatap, G., Royand, F., Blonde-Cynober, F., Aussel, C., \& Vincent, J-P. (2005). Is bioelectrical impedance analysis a tool at bedside, during heat waves to assist geriatricians with discriminative diagnosis of hypertonic dehydration? Journal of Nutrition, Health and Aging, 9(6), 441-445.

Scott, D., Rycroft, J.A., Aspen, J., Chapman, C., \& Brown, B. (2004). The effect of drinking tea at high altitude on hydration status and mood. European Journal of Applied Physiology, 91, 493-498. 
Sergi, G., Lupoli, L. Enzi, G., Volpato, S., Perissinotto, E., Bertani, R., Inelmen, E.M., Bonometto, P., Busetto, L., Berton, A., \& Coin, A. (2006). Reliability of bioelectrical impedance methods in detecting body fluids in elderly patients with congestive heart failure. Scandinavian Journal of Clinical and Laboratory Investigation, 66, 19-30.

Shanholtzer, B.A., \& Patterson, S.M. (2003). Use of bioelectrical impedance in hydration status assessment: reliability of a new tool in psychophysiology research. International Journal of Psychophysiology, 49, 217-226.

Shaw, K.A., Srikanth, V.K., Fryer, J.L., Blizzard, L., Dwyer, T., \& Venn, A.J. (2007). Dual energy X-ray absorptiometry body composition and aging in a populationbased older cohort. International Journal of Obesity, 31, 279-284.

Sheng, H-P. (2006). Body fluids and water balance. In M. Stipanuk (Ed.), Biochemical, physiological, molecular aspects of human nutrition second ed. (pp. 973-1000). St. Louis, Missouri: Saunders Elsevier, Inc.

Shils, M.E. (2006). Appendices. In A. Bloch \& M. Shils (Eds.), Modern nutrition in health and disease, 10th edition (page 1851), Philadelphia, PA: Lippincott Williams \& Wilkins.

Shirrefs, S.M. (2003). Markers of hydration status. European Journal of Clinical Nutrition, 57, S6-S9.

Shirrefs, S.M. (2005). The importance of good hydration for work and exercise performance. Nutrition Reviews, 63(6), S14-S21.

Shirrefs, S.M., Merson, S.J., Fraser, S.M., \& Archer, D.T. (2004). Effect of fluid restriction on hydration status in man. British Journal of Nutrition, 91, 951-958.

Smith, D., Engel, B., Diskin, A.M., Spanel, P., \& Davies, S.J. (2002). Comparative measurements of total body water in healthy volunteers by online breath deuterium measurement and other near-subject methods. American Journal of Clinical Nutrition, 76, 1295-1301.

Sloane, P.D., Ivey, J., Helton, M., Barrick, A.L., \& Cerna, A. (2008). Nutritional issues in long term care. Journal of the American Directors Association, 9, 476-485.

Soo, K., \& Naughton, G. (2007). The hydration profile of female cricket players during competition. International Journal of Sports Nutrition and Exercise Metabolism, $17,14-26$.

Spears, K.E., Kin, H., Behall, K.M., \& Conway, J.M. (2009). Hand-held indirect calorimeter offers advantages compared with prediction equations, in a group of 
overweight women, to determine resting energy expenditure and estimated total energy expenditures during research screening. Journal of the American Dietetic Association, 109, 836-845.

Stachenfeld, N.S., Mack, G.W., Takamata, A., DiPietro, L. \& Nadel, E.R. (1996). Thirst and fluid regulatory responses to hypertonicity in older adults. American Journal of Physiology, 271, R757-R765.

Steijaert, M. Deurenberg, P. Van Gaal, L., \& De Leewu, I. (1997). The use of multifrequency impedance to determine total body water and extracellular water in obese and lean female individuals. International Journal of Obesity, 21, 930-934.

Stookey, J.D., Pieper, C.F., \& Cohen, H.J. (2005). Is the prevalence of dehydration among community-dwelling older adults really low? Informing current debate over the fluid recommendation for adults aged $70+$ years. Public Health Nutrition, $8(8), 1275-1285$.

Svendsen, O.L., Haarbo, J. Hassager, C., \& Christiansen, C. (1993). Accuracy of measurements of body composition by dual-energy X-ray absorptiometry in vivo. American Journal of Clinical Nutrition, 57, 605-608.

Tabachnick, B.G., \& Fidell, L.S. (2007). Using multivariate statistics, Boston, MA: Pearson Education, Inc.

Taivainen, H., Laitinen, R., Tahtela, R., Kiianmaa, K., \& Valimaki, M.J. (1995). Role of plasma vasopressin in changes of water balance accompanying acute alcohol intoxication. Alcoholism, Clinical and Experimental Research, 19, 759-762.

Thomas, D.R., Cote, T.R., Lawhorne, L., Stevenson, S.A., Rubenstein, L.Z., Smith, D.A., Stefanacci, R.G., Tangalos, E.G., \& Morley, J.E. (2008). Understanding clinical dehydration and its treatment. Journal of the American Medical Directors Association, 9, 292-301.

Thomas, D.R., Tariq, S.H., Makhdomm, S., Haddad, R., \& Moinuddin, A. (2004). Physician misdiagnosis of dehydration in older adults. Journal of the American Medical Directors Association, 5, S31-S34.

U.S. Department of Agriculture/Agriculture Research Service. (2002). USDA National Nutrient Database for Standard Reference, Release 21. Retrieved August 3, 2010, from http://www.ars.usda.gov/ba/bhnrc/ndl.

U.S. Department of Health and Human Services (USDHH) and U.S. Department of Agriculture (USDA). Dietary Guidelines for Americans 2010. (2010). Retrieved September 1, 2010, http://www.cnpp.usda.gov/DGAs2010-DGACReport.htm 
Utter, A.C., \& Lambeth, P.G. (2010). Evaluation of multifrequency bioelectrical impedance analysis in assessing body composition in wrestlers. Medicine and Science in Sports and Exercise, 42(2), 361-367.

Valente, S., \& Fisher, D. (2010). Recognizing and Managing Psychogenic Polydipsia in Mental Health. The Journal of Nurse Practitioners, 6(7), 546-550.

Valtin, H. "Drink at least eight glasses of water a day." Really? Is there scientific evidence for "8X8"? (2002). American Journal of Physiology. Regulatory, Integrative and Comparative Physiology, 283, R993-R1004.

Vander Weg, M.W., Watson, J.M., Klesges, R.C., Clemens, L.H., Slawson, D.L., \& McClanaha, B.S. (2004). Development and cross-validation of a prediction equation for estimating resting energy expenditure in healthy African-American and European-American women. European Journal of Clinical Nutrition, 58, $474-480$.

Van Loan, M.D. (1990). Bioelectrical impedance analysis to determine fat-free mass, total body water and body fat. Sports Medicine, 10, 205-217.

Van Loan, M.D. (1998). Estimates of fat-free mass (FFM) by densitometry, dual energy X-ray absorptiometry (DXA), and bioimpedance spectroscopy (BIS) in Caucasian and Chinese-American women. Applied Radiation and Isotopes, 49, 751-752.

Van Loan, M.D., \& Mayclin, P.L. (1992). Body composition assessment: dual-energy Xray absorptiometry (DEXA) compared to reference methods. European Journal of Clinical Nutrition, 46, 125-130.

Van Loan, M.D., \& Mayclin, P.L. (1987). Bioelectrical impedance analysis: Is it a reliable estimator of lean body mass and total body water? Human Biology, 56, 219-226.

Vivanti, A., Harvey, K., \& Ash, S. (2010). Developing a quick and practical screen to improve the identification of poor hydration in geriatric and rehabilitative care. Archives of Gerontology and Geriatrics, 50, 156-164.

Vivanti, A., Harvey, K, Ash, S., \& Battistutta, D. (2008). Clinical assessment of dehydration in older people admitted to hospital. What are the strongest indicators? Archives of Gerontology and Geriatrics, 47, 340-355.

Volaufova, J. (2005). Statistical methods in biomedical research and measurement science. Measurement Science Review, 5, 1-10.

Wakefield, B., Mentes, J., Diggelmann, L., \& Culp, K. (2002). Monitoring hydration status in elderly veterans. Western Journal of Nursing Research, 24(2), 132-142. 
Wakefield, B., Mentes, J., Holman, J.E., \& Culp, K. (2008). Risk factors and outcomes associated with hospital admission for dehydration. Rehabilitation Nursing, 33(6), $233-241$.

Wakefield, B., Mentes, J., Holman, J.E., \& Culp, K. (2009). Post-admission dehydration: risk factors, indicators, and outcomes. Rehabilitation Nursing, 34(5), 209-216.

Walsh, N.P., Laing, S.J., Oliver, S.J., Montague, J.C., Walters, R., \& Bilzon, J.L. (2004). Saliva parameters as potential indices of hydration status during acute dehydration. Medicine and Science in Sports and Exercise, 36(9), 1535-1542.

Walsh, N.P., Montague, J.C., Callow, N., \& Rowlands, A.V. (2004). Saliva flow rate, total protein concentration and osmolality as potential markers of whole body hydration status during progressive acute dehydration in humans. Archives of Oral Biology, 49, 149-154.

Wang, Z.M., Deurenberg, P., Wang, W., Pietrobelli, A., Baumgartner, R.N., \& Heymsfield, S.B. (1999). Hydration of fat-free body mass: review and critique of a classic body-composition constant. American Journal of Clinical Nutrition, 69, 833-841.

Weinberg, A.D., \& Minaker, K.L. (1995). Council of scientific affairs, American Medical Association. Dehydration: Evaluation and management in older adults. Journal of the American Medical Association, 274, 1552-1556.

Wenhold, F., \& Faber, M. (2009). Water in nutritional health of individuals and households: An overview. Water, South Africa., 35, 61-70.

Whelan, K. (2001). Inadequate fluid intakes in dysphagic acute stroke. Clinical Nutrition, 20(5), 423-428.

Whitmire, S.J. (2008). Nutrition-focused evaluation and management of dysnatremias. Nutrition in Clinical Practice, 23, 108-121.

Willits, C.O. (1951). Methods for determination of moisture- oven drying. Analytical Chemistry, 23(8), 1058-1062.

Wotton, K., Crannitch, K., \& Munt, R. (2008). Prevalence, risk factors and strategies to prevent dehydration in older adults. Contemporary Nurse, 31, 45-56.

Wotton, M.J., Thomas, B.J., Cornish, B.H., \& Ward, L.C. (2000). Comparison of whole body and segmental bioimpedance methodologies for estimating total body water. Annals of the New York Academy of Sciences, 904, 181-186. 
Writing group of the nutrition care process/standardized language committee. Nutrition care process and model part I: The 2008 update (2008). Journal of the American Dietetic Association, 108, 1113-1117.

Yamada, Y., Masuo, Y., Yokoyama, K., Hashii, Y., Ando, S., Okayama, Y., Morimoto, T., Kimura, M., \& Oda, S. (2009). Proximal electrode placement improves the estimation of body composition in obese and lean elderly during segmental bioelectrical impedance analysis. European Journal of Applied Physiology, 107, $135-144$.

Yoshihara, A., Hirotomi, T., Takano, N., Kondo, T., Hanada, N., \& Miyazaki, H. (2007). Serum markers of chronic dehydration are associated with saliva spinability. Journal of Oral Rehabilitation, 34, 733-738.

Zamboni, M., Zoico, E., Scartezzini, T., Mazzali, G., Tosoni, P., Zivelonghi, A., Gallagher, D., De Pergola, G., Di Francesco, V., \& Bosello, O. (2003). Body composition changes in stable-weight elderly subjects: The effect of sex. Aging in Clinical and Experimental Research, 15, 321-327.

Zappe, D.H., Bell, G.W., Swartzentruber, H., Wideman, R.F., \& Kenney, W.L. (1996). Age and regulation of fluid and electrolyte balance during repeated exercise sessions. American Journal of Physiology, 270, R71-R79.

Zhao, G., Ford, E.S., \& Modkad, A.H. (2008). Racial/ethnic variation in hypertensionrelated lifestyle behaviors among US women with self-reported hypertension. Journal of Human Hypertension, 22, 608-616.

Zizza, C.A., Ellison, K.J., \& Wernette, C.M. (2009). Total water intakes of communityliving middle-old and oldest-old adults. Journal of Gerontology. Series A, Biological Sciences and Medical Sciences, 64A(4), 481-486. 
VITA

STACEY L. TANNENBAUM

May 1982

1982-1983

1984-1988

January 1987

1988-1990

1990-1992

1995-2004

2006

2006-2011

2006-2007

2007-2008
Bachelor of Science in Nutrition

The Pennsylvania State University

State College, Pennsylvania

Dietetic Internship

Touro Infirmary

New Orleans, Louisiana

Clinical Dietitian

Cabrini Medical Center

New York, New York

Master of Science in Nutrition

New York University

New York, New York

Institutional Sales Representative

Mead Johnson Nutritionals

New York, New York

Clinical Dietitian

Humana Hospital Bennett

Plantation, Florida

Clinical Dietitian

Private Practice

Sunrise, Florida

Adjunct Professor of Nutrition Course

Keiser College

Fort Lauderdale, Florida

Doctoral Student of Philosophy in Nutrition

Florida International University

Miami, Florida

Research Assistant

Florida International University

Miami, Florida

Teaching Assistant 
Florida International University

Miami, Florida

2009

Graduate Assistant

Florida International University

Miami, Florida

\section{HONORS AND AWARDS}

1. Doctoral Evidence Acquisition Fellowship, Florida International University Graduate School, 2009.

2. Dissertation Year Fellowship, Florida International University Graduate School, 2010.

3. American Dietetic Association Foundation Scholarship, 2010.

\section{PRESENTATIONS AND PUBLICATIONS}

1. Medicine and Society interdisciplinary approach to health care - expert panelist in nutrition among a panel of physicians and allied health specialists. College of Medicine, Florida International University, Miami, FL, October 2009.

2. Tannenbaum SL, Castellanos VH, Arheart KL. Comparison of estimated water requirements of adults across age groups, American Public Health Association, Philadelphia, PA, November 2009.

3. Caban-Martinez A, Davila EP, Lee DJ, Fleming, LE, LeBlanc W, Arheart K, McCollister KE, Christ S, Tannenbaum SL. Oral water intake across major US industries: NHANES 1999-2004, American Public Health Association, Philadelphia, PA, November 2009.

4. Tannenbaum SL, Castellanos VH, Arheart KL. Comparison of water intake in milliliters/kilocalorie consumed across gender and age groups, American Society of Enteral and Parenteral Nutrition, Clinical Nutrition Week, Las Vegas, Nevada, February 2010.

5. Tannenbaum SL, Castellanos VH, Arheart KL. Comparison of estimated water requirements of adults across BMI and gender groups: NHANES 1999-2004, American Public Health Association, Denver, CO, November 2010. 